\title{
On bounded pseudodifferential operators in Wiener spaces
}

\author{
Laurent Amour, Lisette Jager and Jean Nourrigat \\ Université de Reims
}

\begin{abstract}
We aim at extending the definition of the Weyl calculus to an infinite dimensional setting, by replacing the phase space $\mathbb{R}^{2 n}$ by $B^{2}$, where $(i, H, B)$ is an abstract Wiener space. A first approach is to generalize the integral definition using the Wigner function. The symbol is then a function defined on $B^{2}$ and belonging to a $L^{1}$ space for a gaussian measure, the Weyl operator is defined as a quadratic form on a dense subspace of $L^{2}(B)$. For example, the symbol can be the stochastic extension on $B^{2}$, in the sense of L. Gross, of a function $F$ which is continuous and bounded on $H^{2}$.

In the second approach, this function $F$ defined on $H^{2}$ satisfies differentiability conditions analogous to the finite dimensional ones. One needs to introduce hybrid operators acting as Weyl operators on the variables of finite dimensional subset of $H$ and as Anti-Wick operators on the rest of the variables. The final Weyl operator is then defined as a limit and it is continuous on a $L^{2}$ space. Under rather weak conditions, it is an extension of the operator defined by the first approach.

We give examples of monomial symbols linking this construction to the classical pseudodifferential operators theory and other examples related to other fields or previous works on this subject.
\end{abstract}

\section{Contents}

1 Introduction $\quad 2$

2 Anti-Wick, Weyl and hybrid operators. 7

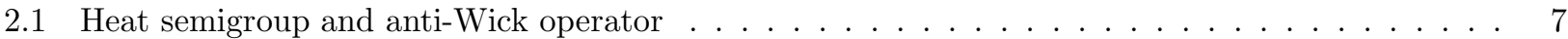

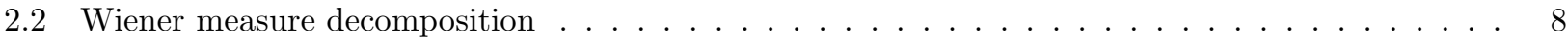

2.3 Partial heat semigroup and hybrid operators $\ldots \ldots \ldots \ldots \ldots \ldots$

3 Plan of the proof of Theorem 1.4

4 Some useful operators in Wiener spaces 11

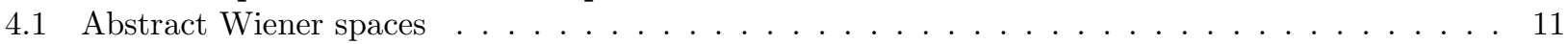

4.2 Stochastic extensions . . . . . . . . . . . . . . . . . . . . . . . 13

4.3 The Heat operator (continued) . . . . . . . . . . . . . . . . . . . . . . . . . 14

4.4 Coherent states and Segal Bargmann transformation . . . . . . . . . . . . . . . . . . 16

4.5 Wigner Gaussian function . . . . . . . . . . . . . . . . . . . . . . . . . . 18

4.6 Convergence in Definition $1.2 \ldots \ldots$. . . . . . . . . . . . . . . . . . . . . . . . . . 20

4.7 Anti-Wick and hybrid operators . . . . . . . . . . . . . . . . . . . . . . 21

4.8 Partial Heat semigroups and stochastic extensions. . . . . . . . . . . . . . . . . . . 22

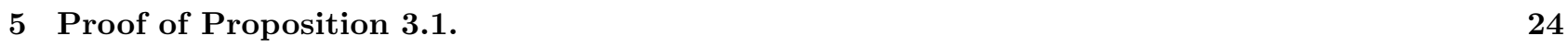

6 Proof of Proposition $3.3 . \quad 28$

7 Wick symbol of a Weyl operator $\quad \lcm{30}$ 


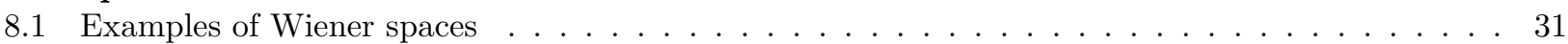

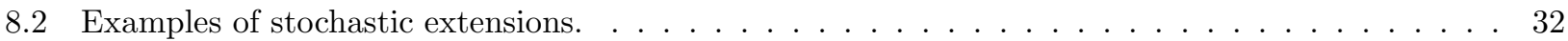

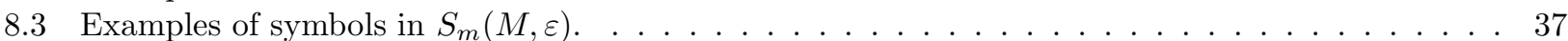

\section{Introduction}

The Weyl calculus associates with every function $F$, called symbol, bounded and measurable on $\mathbb{R}^{2 n}$ and every $h>0$ an operator, denoted by $O p_{h}^{W e y l, L e b}(F)$, from the Schwartz space $\mathcal{S}\left(\mathbb{R}^{n}\right)$ into its dual space $\mathcal{S}^{\prime}\left(\mathbb{R}^{n}\right)$. For a function $f$ belonging to $\mathcal{S}\left(\mathbb{R}^{n}\right)$, this operator is, formally, defined by

$$
\left(O p_{h}^{W e y l, L e b}(F) f\right)(x)=(2 \pi h)^{-n} \int_{\mathbb{R}^{2 n}} e^{\frac{i}{h}(x-y) \cdot \xi} F\left(\frac{x+y}{2}, \xi\right) f(y) d \lambda(y, \xi)
$$

where $\lambda$ is the Lebesgue measure. If $F$ is $C^{\infty}$ and bounded on $\mathbb{R}^{2 n}$ as well as all its derivatives, then CalderónVaillancourt's theorem states that $O p_{h}^{W e y l, L e b}(F)$ extends to a bounded operator on $L^{2}\left(\mathbb{R}^{n}, \lambda\right)$ (see [C-V], [HO]).

The definition of the Weyl operator as an application on $\mathcal{S}\left(\mathbb{R}^{n}\right)$ with values in $\mathcal{S}^{\prime}\left(\mathbb{R}^{n}\right)$ or, equivalently, as a quadratic form on $\mathcal{S}\left(\mathbb{R}^{n}\right)$, has already been extended to the infinite-dimensional case for some specific symbols by Kree-Rạczka [K-R] and, up to a small modification, by Bernard Lascar ([LA-1]), (see [LA-2] to [LA-10] as well for applications). In the present paper the hypotheses on the symbol of the operator (the function $F$ in (1)) are weaker than by these authors. We also give a Calderón-Vaillancourt type result in this context.

The classical definition (1) does not lend itself to an extension to an infinite dimensional case. We shall use instead the definition of $O p_{h}^{W e y l, L e b}(F)$ in which the Wigner function appears. This operator is the only one that satisfies, for all $f$ and $g$ in $\mathcal{S}\left(\mathbb{R}^{n}\right)$ :

$$
<O p_{h}^{W e y l, L e b}(F) f, g>_{L^{2}\left(\mathbb{R}^{n}, \lambda\right)}=(2 \pi h)^{-n} \int_{\mathbb{R}^{2 n}} F(Z) H_{h}^{L e b}(f, g, Z) d \lambda(Z),
$$

where $H_{h}^{L e b}(f, g,$.$) is the Wigner function (for the Lebesgue measure):$

$$
H_{h}^{L e b}(f, g, Z)=\int_{\mathbb{R}^{n}} e^{-\frac{i}{h} t \cdot \zeta} f\left(z+\frac{t}{2}\right) \overline{g\left(z-\frac{t}{2}\right)} d \lambda(t) \quad Z=(z, \zeta) \in \mathbb{R}^{2 n}
$$

(cf Unterberger [U-2], or Lerner [LE], sections 2.1.1 and 2.1.2, or Combescure Robert [C-R], section 2.2).

The expression (2) is well defined for $f$ and $g$ in $\mathcal{S}\left(\mathbb{R}^{n}\right)$ even if $F$ is only supposed to be measurable and bounded. Indeed, the Wigner function $H_{h}^{L e b}(f, g, \cdot)$ is in $\mathcal{S}\left(\mathbb{R}^{2 n}\right)$. We first extend this definition (2) to the infinite dimensional case (using Wiener spaces) in the same spirit, by defining the operator as a bilinear form on a convenient dense subspace, with very weak assumptions on $F$ (weaker than those of [LA-1] and [K-R] : see definition 1.2 below. To this aim we associate a Gaussian Wigner function $H_{h}^{\text {Gauss }}(f, g)$ with every couple $(f, g)$, where $f$ and $g$ are convenient functions defined on a Wiener space. Then we prove that, under CalderónVaillancourt type conditions, this operator extends to a bounded operator on a $L^{2}$ space (Theorem 1.4, which is the main result).

We successively indicate what replaces the space $\mathbb{R}^{n}$, the Lebesgue measure, the Schwartz's space $\mathcal{S}\left(\mathbb{R}^{n}\right)$ and the Wigner function. Then we shall be ready to define the Weyl operator as a quadratic form, as in (2).

The space $\mathbb{R}^{n}$ is replaced by a real separable infinite-dimensional Hilbert space $H$, the configuration space. The symbol $F$ is a function on the phase space $H^{2}$. One denotes by $a \cdot b$ the scalar product of two elements $a$ and $b$ of $H$ and by $|a|$ the norm of an element $a$ of $H$. 
The Lebesgue measure is replaced by a Gaussian measure associated with the norm of $H$. But, since $H$ is infinite-dimensional, this Gaussian measure will be a measure, not on $H$ (which is impossible) but on a convenient Banach space $B$ containing $H$. Abstract Wiener spaces are an adequate frame. An abstract Wiener space is a triple $(i, H, B)$, where $H$ is a real, separable Hilbert space, $B$ a Banach space and $i$ a continuous injection from $H$ into $B$ such that $i(H)$ is dense in $B$, other conditions being satisfied (see [G-2, G-3, $\mathrm{K}$ ] or Definition 4.1). The injection $i$ is generally not mentioned and one usually identifies $H$ with its dual space, so that the preceding hypotheses imply

$$
B^{\prime} \subset H^{\prime}=H \subset B,
$$

where every space is continuously embedded as a dense subspace of the following one. For every $u$ in $B^{\prime}$ and $x$ in $B$, one denotes by $u(x)$ the duality between these elements and one supposes that, if $x$ is in $H, u(x)=u \cdot x$, This will be the case in the rest of the article.

One denotes by $\mathcal{F}(H)$ (resp. $\mathcal{F}\left(B^{\prime}\right)$ ) the set of all finite-dimensional subspaces $E$ of $H$ (resp. of $B^{\prime}$ ). We note that $\mathcal{F}\left(B^{\prime}\right) \subset \mathcal{F}(H)$ by (4). For every $E$ belonging to $\mathcal{F}(H)$ and every positive $h$, one defines a probability measure $\mu_{E, h}$ on $E$ by:

$$
d \mu_{E, h}(x)=(2 \pi h)^{-(1 / 2) \operatorname{dim}(E)} e^{-\frac{|x|^{2}}{2 h}} d \lambda(x),
$$

where $\lambda$ is the Lebesgue measure on $E$, the norm on $E$ being the restriction to $E$ of the norm of $H$. If $B$ is a Banach space satisfying (4), the continuity and density conditions being satisfied, one defines, for each $E$ is $\mathcal{F}\left(B^{\prime}\right)$, an application $P_{E}: B \rightarrow E$ by:

$$
P_{E}(x)=\sum_{j=1}^{n} u_{j}(x) u_{j},
$$

where $\left\{u_{1}, \ldots, u_{n}\right\}$ is a basis of $E$, orthonormal for the restriction to $E$ of the scalar product of $H$. This application does not depend on the choice of the basis. If the Banach space $B$ satisfies proper conditions, (see Definition 4.1 for precisions), for each positive $h$, one can derive a probability measure $\mu_{B, h}$ on the Borel $\sigma$-algebra of $B$, with the following property. For every $E$ in $\mathcal{F}\left(B^{\prime}\right)$ and every function $\varphi$ in $L^{1}\left(E, \mu_{E, h}\right)$, the function $\varphi \circ P_{E}$ is in $L^{1}\left(B, \mu_{B, h}\right)$, and one has

$$
\int_{B}\left(\varphi \circ P_{E}\right)(x) d \mu_{B, h}(x)=\int_{E} \varphi(y) d \mu_{E, h}(y) .
$$

Other properties of the measure $\mu_{B, h}$ are recalled in section 4.1 and examples are given in Section 8 , In Section 4.2, the same notions will be seen for subspaces $E$ in $\mathcal{F}(H)$. The Banach space $B$ associated with $H$ is not unique but for any choice of $B$ and any positive $h$, the space $L^{2}\left(B, \mu_{B, h}\right)$ is isomorphic to the symmetrized Fock space $\mathcal{F}_{s}(H)$, which does not depend on the choice of $B$ (cf [F] or [A-J-N-1]). If the Hilbert space $H$ is finite dimensional, then $B=H$. If not, $B$ is sometimes derived from $H$ thanks to a Hilbert-Schmidt operator (cf [G-3], Example 2, p. 192)), but other constructions are possible. In Section, 8 examples related to Brownian motion, field theory and interacting lattices will be given.

Now let us introduce the space which will replace $\mathcal{S}\left(\mathbb{R}^{n}\right)$ in (2) (3). For every $E$ in $\mathcal{F}(H)$, we shall need the isometric isomorphism $\gamma_{E, h / 2}$ between $L^{2}\left(E, \mu_{E, h / 2}\right)$ and $L^{2}(E, \lambda)$ given by

$$
\left(\gamma_{E, h / 2} \varphi\right)(x)=(\pi h)^{-(1 / 4) \operatorname{dim}(E)} \varphi(x) e^{-\frac{|x|^{2}}{2 h}} \quad \varphi \in L^{2}\left(E, \mu_{E, h / 2}\right) .
$$

One denotes by $\mathcal{S}_{E}$ the space of all functions $\varphi: E \rightarrow \mathbf{C}$ such that $\gamma_{E, h / 2} \varphi$ belongs to the Schwartz space of rapidly decreasing functions $\mathcal{S}(E)$. For $E \in \mathcal{F}\left(B^{\prime}\right)$, let $\mathcal{D}_{E}$ be the set of applications $f: B \rightarrow \mathbf{C}$ of the form $f=\varphi \circ P_{E}$, where $P_{E}: B \rightarrow E$ is defined by (6) and $\varphi: E \rightarrow \mathbf{C}$ belongs to $\mathcal{S}_{E}$. We denote by $\mathcal{D}$ the union of the spaces $\mathcal{D}_{E}$, taken over all $E$ in $\mathcal{F}\left(B^{\prime}\right)$. This space $\mathcal{D}$ is dense in $L^{2}\left(B, \mu_{B, h / 2}\right)$. Indeed, if $\left(e_{j}\right)_{(j \geq 1)}$ is a Hilbert basis of $H$, the vectors $e_{j}$ belonging to $B^{\prime}$, the set of functions on $B$ which are polynomials of a finite number of functions $x \rightarrow e_{j}(x)$ is contained in $\mathcal{D}$ and is dense in $L^{2}\left(B, \mu_{B, h / 2}\right)$ (see for example [A-J-N-1]).The constant functions belong to $\mathcal{D}_{E}$ for every $E$ in $\mathcal{F}\left(B^{\prime}\right)$.

We very often need the following classical result (Kuo, $[\mathrm{K}]$, Chapter 1 Section 4). 


\section{Proposition 1.1.}

1. The space $B^{\prime}$ is contained in $L^{2}\left(B, \mu_{B, h}\right)$ and the norm of $u \in B^{\prime}$, considered as an element of $L^{2}\left(B, \mu_{B, h}\right)$, is equal to $\sqrt{h}|u|$.

2. The inclusion of $B^{\prime}$ into $L^{2}\left(B, \mu_{B, h}\right)$ extends to a continuous linear map from $H$ into $L^{2}\left(B, \mu_{B, h}\right)$, with norm $\sqrt{h}$, denoted by $u \rightarrow \ell_{u}$.

The first point can be seen by applying (17) to a one dimensional space $E$. The map $\ell$ turns $H$ into a Gaussian space in the sense of [J] or into a "Gaussian random process", in the sense of [SI]. In the case of Example 8.2, for every $u$ in the Cameron-Martin space, $\ell_{u}$ is the Itô integral of the function $u^{\prime}$.

Let us now define the Wigner-Gauss function, which will replace the usual Wigner function. Let $(i, H, B)$ be a Wiener space satisfying (4). For every subspace $E$ in $\mathcal{F}(H)$, for all $\varphi$ and $\psi$ in $\mathcal{S}_{E}$, one defines a function $\widehat{H}_{h}^{\text {Gauss }}(\varphi, \psi)$ on $E^{2}$, setting:

$$
\widehat{H}_{h}^{\text {Gauss }}(\varphi, \psi)(z, \zeta)=e^{\frac{|\zeta|^{2}}{h}} \int_{E} e^{-2 \frac{i}{h} \zeta \cdot t} \varphi(z+t) \overline{\psi(z-t)} d \mu_{E, h / 2}(t) \quad(z, \zeta) \in E^{2} .
$$

One notices that, for $Z$ in $E^{2}$ :

$$
\widehat{H}_{h}^{\text {Gauss }}(\varphi, \psi)(Z)=2^{-\operatorname{dim}(E)} e^{\frac{|Z|^{2}}{h}} H_{h}^{L e b}\left(\gamma_{E, h / 2} \varphi, \gamma_{E, h / 2} \psi\right)(Z) \quad Z \in E^{2} .
$$

One will see (Proposition 4.8) that this function belongs to $L^{1}\left(E^{2}, \mu_{E^{2}, h / 2}\right)$ and to $L^{2}\left(E^{2}, \mu_{E^{2}, h / 4}\right)$ as well. For all functions $f$ and $g$ in $\mathcal{D}_{E},\left(E\right.$ in $\left.\mathcal{F}\left(B^{\prime}\right)\right)$, of the form $f=\widehat{f} \circ P_{E}$ et $g=\widehat{g} \circ P_{E}$, with $\widehat{f}$ and $\widehat{g}$ in $\mathcal{S}_{E}$, one defines the Wigner-Gauss transform $H_{h}^{\text {Gauss }}(f, g)$, which is the function defined on $E^{2}$ by:

$$
H_{h}^{\text {Gauss }}(f, g)(Z)=\widehat{H}_{h}^{\text {Gauss }}(\widehat{f}, \widehat{g})\left(P_{E} Z\right) \quad \text { a.e } Z \in B^{2} .
$$

One writes $P_{E}$ instead of $P_{E^{2}}$ for the sake of simplicity. One will see in Section 4.2 how to modify this definition if $E \in \mathcal{F}(H)$. According to (77), it follows from Proposition 4.8 that this function is in $L^{1}\left(B^{2}, \mu_{B^{2}, h / 2}\right)$ and $L^{2}\left(B^{2}, \mu_{B^{2}, h / 4}\right)$. If $f$ and $g$ are in $\mathcal{D}$, the subspace $E$ such that $f$ and $g$ are in $\mathcal{D}_{E}$ is not unique, but the function defined above does not depend on $E$. Proposition 4.8 states that this Wigner-Gauss transformation extends, by density, from $L^{2}\left(B, \mu_{B, h / 2}\right) \times L^{2}\left(B, \mu_{B, h / 2}\right)$ to $L^{2}\left(B^{2}, \mu_{B^{2}, h / 4}\right)$ and to the space of continuous functions defined on $H^{2}$. One will see, in (76), another expression of $H_{h}^{\text {Gauss }}(f, g)$, using Segal-Bargmann transforms of $f$ and $g$.

Now we are almost ready to define the Weyl operator associated with a symbol $F$. If $(i, H, B)$ is a Wiener space, we have the choice of two phase spaces: $H^{2}$ an $B^{2}$. The first one is equipped with the symplectic form $\sigma((x, \xi),(y, \eta))=y \cdot \xi-x \cdot \eta$, but not with a measure adapted to the integrations we want to conduct. On the contrary, the space $B^{2}$ is equipped with the Gaussian measure $\mu_{B^{2}, h}$, but it is not a symplectic space.

This difficulty is overcome in the following way. The symbols will be initially defined as functions on $H^{2}$. There exists an operation, introduced by L. Gross (G-1], see Ramer [RA]) and usually called stochastic extension, which associates with a Borel function $F$ on $H$ or $H^{2}$ a Borel function $\widetilde{F}$ on $B$ or on $B^{2}$. This stochastic extension, which is not a genuine extension, will be recalled in Definition 4.4. What will appear in the initial definition formula (13) of the Weyl operators is the stochastic extension $\widetilde{F}$ of the initial symbol $F$.

In the second step, concerned with the bounded extension in $L^{2}\left(B, \mu_{B, h / 2}\right)$ of this initial operator, we shall restrict ourselves to bounded symbols. But in the initial definition of the operator as a quadratic form on $\mathcal{D}$, a polynomial growth will be enough. For the initial definition (13), the function $\widetilde{F}$ (stochastic extension of the symbol $F$ ) will be in $L^{1}\left(B^{2}, \mu_{B^{2}, h / 2}\right)$ and the polynomial growth will be expressed in terms of the existence of a nonnegative integer $m$ such that the following norm is defined.

$$
N_{m}(\widetilde{F})=\sup _{Y \in H^{2}} \frac{\|\widetilde{F}(\cdot+Y)\|_{L^{1}\left(B^{2}, \mu_{B^{2}, h / 2}\right)}}{(1+|Y|)^{m}}
$$


This norm is finite if the function $F$ is bounded or if it is a polynomial expression of degree $m$ with respect to functions $(x, \xi) \rightarrow \ell_{a}(x)+\ell_{b}(\xi)$, with $a$ and $b$ in $H$.

Definition 1.2. Let $(i, H, B)$ be an abstract Wiener space satisfying (4) and $h>0$. Let $\widetilde{F}$ be a function in $L^{1}\left(B^{2}, \mu_{B^{2}, h / 2}\right)$. Suppose there exists $m \geq 0$ such that the norm $N_{m}(\widetilde{F})$ is finite. We define a quadratic form $Q^{W e y l}(\widetilde{F})$ on $\mathcal{D} \times \mathcal{D}$ in the following way. For all $f$ and $g$ in $\mathcal{D}$, one sets:

$$
Q_{h}^{W e y l}(\widetilde{F})(f, g)=\int_{B^{2}} \widetilde{F}(Z) H_{h}^{\text {Gauss }}(f, g)(Z) d \mu_{B^{2}, h / 2}(Z)
$$

where $H_{h}^{\text {Gauss }}(f, g)$ is defined in (11).

The convergence will be proved in Proposition 4.10, If $\widetilde{F}$ is bounded, the convergence is a consequence of Proposition 4.8 since $H_{h}^{\text {Gauss }}(f, g)$ is in $L^{1}\left(B^{2}, \mu_{B^{2}, h / 2}\right)$. If we only suppose that $N_{m}(\widetilde{F})$ is finite, other arguments are necessary.

One sees the relationship with the classical definition (2), (3). If $F$ has the form $F=\widehat{F} \circ P_{E}$, where $E$ is in $\mathcal{F}\left(B^{\prime}\right)$ and $\widehat{F}$ is a measurable bounded function on $E^{2}$, if $f=\widehat{f} \circ P_{E}$ and $g=\widehat{g} \circ P_{E}$, where $\widehat{f}$ et $\widehat{g}$ are in $\mathcal{S}_{E}$, one has

$$
Q_{h}^{W e y l}\left(\widehat{F} \circ P_{E}\right)\left(\widehat{f} \circ P_{E}, \widehat{g} \circ P_{E}\right)=<O p_{h}^{W e y l, L e b}(\widehat{F}) \gamma_{E, h / 2} \widehat{f}, \gamma_{E, h / 2} \widehat{g}>_{L^{2}(E, \lambda)}
$$

where $O p_{h}^{W e y l, L e b}(\widehat{F})$ is defined on $E$ as it is in (2), (3) on $\mathbb{R}^{n}$. This equality comes from (10).

One can compare this definition with the definition in $\mathrm{K}-\mathrm{R}$ or [LA-1. The authors define an anti-Wick operator associated with a symbol, a function defined on $H^{2}$ which is, for example, the Fourier transform of a complex measure bounded on $H^{2}$. In $[\mathrm{K}-\mathrm{R}$. they associate, too, with such a symbol, a Weyl operator defined as a quadratic form on a dense subspace. When the symbol $F$ is the Fourier transform of a complex measure bounded on $H^{2}$, we prove, in Proposition 8.3 , that $F$ admits a stochastic extension $\widetilde{F}$, with which Definition 1.2 above associates a Weyl operator, defined as a quadratic form. For this kind of symbols, the Weyl operator (as a quadratic form) is explicitly written in (139) and (135) or, equivalently, in (139) and (141). This last form can be found in $[\mathrm{K}-\mathrm{R}]$ as well. The anti-Wick operator can be found in [K-R, LA-1] in the form (140)-(135). Our Definition 1.2 is more general, insofar as admitting a stochastic extension is more general than being the Fourier transform of a bounded measure. Section 8.2 gives other examples of classes of functions admitting stochastic extensions.

However, all functions do not have a stochastic extension. If $H$ is infinite dimensional, the norm function, which associates with every $x \in H$ its norm $|x|$, admits no stochastic extension (see Kuo, $\mathbf{K}$, Chapter 1, section 4). In the same way, the function $x \rightarrow e^{-|x|^{2}}$, defined on $H$, has no stochastic extension. We shall not be able to define a pseudodifferential operator, whose symbol would be $F(x, \xi)=e^{-|x|^{2}}$. This operator would have to be the multiplication by $e^{-|x|^{2}}$, but this function, lacking an extension, is only defined on a negligible set (the Hilbert space $H$ is negligible in $B$, cf $[\mathrm{K}]$ ) and this does not make sense. On the contrary, the function $t \rightarrow e^{-(A t) \cdot t}$, where the operator $A$ is positive, symmetrical and trace-class on $H$, has a stochastic extension (see Proposition 8.7).

It now remains to extend the bilinear form defined above on the subspace $\mathcal{D}$ to get a linear operator bounded on $L^{2}\left(B, \mu_{B, h / 2}\right)$. When the symbol is the Fourier transform of a measure bounded on $H^{2}$ (case treated in $[\mathrm{K}-\mathrm{R}]$ ), the upper bound on the norm is a consequence of Proposition 8.10. For other cases we must specify the hypotheses on $F$, which will strongly depend on the choice of a Hilbert basis of $H$. We can now state the hypothesis on the function $F$, which will be the symbol in our version of the Calderón-Vaillancourt theorem.

Definition 1.3. Let $(i, H, B)$ be an abstract Wiener space satisfying (4). The norm of $H$ will hereafter be denoted by $|\cdot|$ and the scalar product of two elements $a$ and $b$ of $H$ will be denoted by $a \cdot b$. The norm of an element of $H^{2}$ is denoted by $|\cdot|$ as well. For all $X=(x, \xi)$ and $Y=(y, \eta)$ in $H^{2}$, we $\operatorname{set} \sigma(X, Y)=y \cdot \xi-x \cdot \eta$. We 
choose a Hilbert basis $\left(e_{j}\right)_{(j \in \Gamma)}$ of $H$, each vector belonging to $B^{\prime}$, indexed by a countable set $\Gamma$. Set $u_{j}=\left(e_{j}, 0\right)$ and $v_{j}=\left(0, e_{j}\right)(j \in \Gamma)$. A multi-index is a map $(\alpha, \beta)$ from $\Gamma$ into $\mathbb{N} \times \mathbb{N}$ such that $\alpha_{j}=\beta_{j}=0$ except for a finite number of indices. Let $M$ be a nonnegative real number, $m$ a nonnegative integer and $\varepsilon=\left(\varepsilon_{j}\right)_{(j \in \Gamma)}$ a family of nonnegative real numbers. One denotes by $S_{m}(M, \varepsilon)$ the set of bounded continuous functions $F: H^{2} \rightarrow \mathbf{C}$ satisfying the following condition. For every multi-index $(\alpha, \beta)$ such that $0 \leq \alpha_{j} \leq m$ and $0 \leq \beta_{j} \leq m$ for all $j \in \Gamma$, the following derivative

$$
\partial_{u}^{\alpha} \partial_{v}^{\beta} F=\left[\prod_{j \in \Gamma} \partial_{u_{j}}^{\alpha_{j}} \partial_{v_{j}}^{\beta_{j}}\right] F
$$

is well defined, continuous on $H^{2}$ and satisfies, for every $(x, \xi)$ in $H^{2}$

$$
\left|\left[\prod_{j \in \Gamma} \partial_{u_{j}}^{\alpha_{j}} \partial_{v_{j}}^{\beta_{j}}\right] F(x, \xi)\right| \leq M \prod_{j \in \Gamma} \varepsilon_{j}^{\alpha_{j}+\beta_{j}} .
$$

The choice of indexing the basis $\left(e_{j}\right)$ by an arbitrary countable set $\Gamma$ is motivated by possible applications in lattice theory. Cordes [C], Coifman Meyer [C-M] (for the standard quantization, not for the Weyl one) and Hwang [HW] remarked that, in the Calderón-Vaillancourt bounds, it is enough to consider multi-indices $(\alpha, \beta)$ such that $0 \leq \alpha_{j} \leq 1$ et $0 \leq \beta_{j} \leq 1$ for every $j$, which inspired the definition of $S_{m}(M, \varepsilon)$. One finds in Section 8 examples of functions of $S_{m}(M, \varepsilon)$ coming from interacting lattices models.

The main result can be stated as follows.

Theorem 1.4. Let $(i, H, B)$ be an abstract Wiener space satisfying (4) and $h$ be a positive number. Let $\left(e_{j}\right)_{(j \in \Gamma)}$ be a Hilbert space basis of $H$, each vector belonging to $B^{\prime}$, indexed by a countable set $\Gamma$. Let $F$ be a function on $H^{2}$ satisfying the following two hypotheses.

(H1) The function $F$ belongs to the class $S_{2}(M, \varepsilon)$ of Definition 1.3, where $M$ is a nonnegative real number and $\varepsilon=\left(\varepsilon_{j}\right)_{(j \in \Gamma)}$ a square summable family of nonnegative real numbers.

(H2) We assume that $F$ has a stochastic extension $\widetilde{F}$ with respect to both measures $\mu_{B^{2}, h}$ and $\mu_{B^{2}, h / 2}$ (see Definition 4.4.

Then there exists an operator, denoted by $O p_{h}^{W e y l}(F)$, bounded in $L^{2}\left(B, \mu_{B, h / 2}\right)$, such that, for all $f$ et $g$ in $\mathcal{D}$

$$
<O p_{h}^{W e y l}(F) f, g>=Q_{h}^{W e y l}(\widetilde{F})(f, g),
$$

where the right hand side is defined by Definition [1.2. Moreover, if $h$ is in $(0,1]$ :

$$
\left\|O p_{h}^{W e y l}(F)\right\|_{\mathcal{L}\left(L^{2}\left(B, \mu_{B, h / 2}\right)\right)} \leq M \prod_{j \in \Gamma}\left(1+81 \pi h S_{\varepsilon} \varepsilon_{j}^{2}\right),
$$

where

$$
S_{\varepsilon}=\sup _{j \in \Gamma} \max \left(1, \varepsilon_{j}^{2}\right) .
$$

One will see in Proposition 8.4 that, if a function $F$ is in $S_{1}(M, \varepsilon)$ and if the sequence $\varepsilon=\left(\varepsilon_{j}\right)_{(j \in \Gamma)}$ is summable, then $F$ satisfies the hypothesis (H2). Proposition 8.11 gives an example of function in $S_{m}(M, \varepsilon)$, inspired by the lattice theory.

The operator $O p_{h}^{W e y l}(F)$ associated with $F \in S_{2}(M, \varepsilon)$ will not be defined by an integral expression, but as the limit, in $\mathcal{L}\left(L^{2}\left(B, \mu_{B, h / 2}\right)\right)$, of a sequence of operators. In order to define this sequence we shall associate with each subspace $E$ in $\mathcal{F}\left(B^{\prime}\right)$, an operator denoted by $O p_{h}^{h y b, E}(\widetilde{F})$ and bounded on $L^{2}\left(B, \mu_{B, h / 2}\right)$. This operator behaves as a Weyl operator on a set of variables and as an anti-Wick operator on the other variables. It will have the form $Q_{h}^{W e y l}(G)$, where $G$ is obtained from the extension $\widetilde{F}$ by applying a partial heat operator 
concerning the variables in $E^{\perp}$ (see Section 2). Then we shall prove that, if one replaces $E$ by a sequence $E\left(\Lambda_{n}\right)=\operatorname{Vect}\left(e_{j}\right)_{\left(j \in \Lambda_{n}\right)}$ where $\left(\Lambda_{n}\right)$ is an increasing sequence of finite subsets of $\Gamma$ whose union is $\Gamma$, then the sequence of operators $\left(O p_{h}^{h y b, E\left(\Lambda_{n}\right)}(G)\right)_{n}$ is a Cauchy sequence in $\mathcal{L}\left(L^{2}\left(B, \mu_{B, h / 2}\right)\right)$. Its limit will be denoted by $O p_{h}^{W e y l}(F)$. We shall see that, if one restricts this operator to get a bilinear form on $\mathcal{D}$, it coincides with the one defined by Definition 1.2. In particular it does not depend on the Hilbert basis $\left(e_{j}\right)$ chosen to construct it, nor on the sequence $\left(\Lambda_{n}\right)$.

The hybrid operator associated with each finite dimensional subspace is defined in Section 2. Section 3 presents more precisely the main steps of the proof of Theorem 1.4. Section 4 first recalls classical facts about Wiener spaces, then gives a precise definition of the stochastic extensions, recalls the Segal Bargmann transformation, gives the necessary upper bounds about the Gaussian Wigner function and gives another expression of the Weyl and hybrid operators, more convenient to get norm estimates. Section 5 establishes the norm estimates which prove that the sequence constructed to approach $O p_{h}^{W e y l}(F)$ is indeed a Cauchy sequence. In Section 6] we prove that $O p_{h}^{W e y l}(F)$ is really an extension of the initially defined operator (Definition 1.2). Section 7 studies the Wick symbol of this operator. Section 8 gives examples of Wiener spaces, stochastic extensions and symbols belonging to $S_{m}(M, \varepsilon)$.

This paper would not have been written without a workgroup organized by F. Hérau, who induced us strongly to get acquainted with Wiener spaces.

\section{Anti-Wick, Weyl and hybrid operators.}

\subsection{Heat semigroup and anti-Wick operator}

In an Euclidean finite dimensional space $E$ one may associate with any Borel bounded function $F$ on $E^{2}$, and for all $h>0$, an operator $O p_{h}^{A W, L e b}(F)$ called the anti-Wick or Berezin-Wick operator. Instead of referring to the usual definition, let us say that it is the only operator satisfying

$$
<O p_{h}^{A W, L e b}(F) f, g>_{L e b}=<O p_{h}^{W e y l, L e b}\left(e^{\frac{h}{4} \Delta} F\right) f, g>_{L e b}
$$

for all $f$ and $g$ in the Schwartz space $\mathcal{S}(E)$. This operator has a bounded extension in $L^{2}(E, \lambda)$, also denoted by $O p_{h}^{A W, L e b}(F)$, which verifies

$$
\left\|O p_{h}^{A W, L e b}(F)\right\|_{\mathcal{L}\left(L^{2}(E, \lambda)\right)} \leq\|F\|_{L^{\infty}\left(E^{2}\right)} .
$$

This property is easier to extend and hence is more convenient as a starting point than the usual definition. See [F] or [LE], Chapter 2.

To extend Definition (20) to the finite dimensional setting, we need an analogue of the heat semigroup on $B^{2}$, that is the family of operators $\widetilde{H}_{t}$ defined by:

$$
\left(\widetilde{H}_{t} F\right)(X)=\int_{B^{2}} F(X+Y) d \mu_{B^{2}, t}(Y) \quad X \in B^{2} .
$$

The operator $\widetilde{H}_{t}$ corresponds, in the finite dimensional case, to the operator $e^{\frac{t}{2} \Delta}$. For every $t>0$, the operator $\widetilde{H}_{t}$ is bounded in the space of all bounded Borel functions on $B^{2}$ (see Hall [HA]). Moreover, it is bounded and with a norm smaller than 1 from $L^{p}\left(B^{2}, \mu_{B^{2}, t+h}\right)$ in $L^{p}\left(B^{2}, \mu_{B^{2}, h}\right)(1 \leq p<\infty, t>0, h>0)$, see Proposition 4.5 below.

For every positive $h$, one associates with each bounded Borel function $F$ on $B^{2}$ an operator called anti-Wick operator. It will first be defined as a quadratic form on $\mathcal{D} \times \mathcal{D}$, denoted by $Q_{h}^{A W}(F)$ and such that

$$
Q_{h}^{A W}(F)(f, g)=Q_{h}^{W e y l}\left(\widetilde{H}_{h / 2} F\right)(f, g),
$$


where the Weyl quadratic form is the one of Definition 1.2. One will see in Corollary 4.12 an expression which is closer to the usual definition, as well as the fact that this quadratic form is linked with a bounded operator, whose norm will be specified.

The following step is associating, with every subspace $E$ in $\mathcal{F}\left(B^{\prime}\right)$, a hybrid operator, with the help of a partial heat operator. This hybrid operator behaves as a Weyl operator associated with $F$ regarding some functions, and as an anti-Wick operator associated with the same symbol regarding some other functions.

\section{$2.2 \quad$ Wiener measure decomposition}

The following proposition proved in Gross [G-4] or in [RA] allows to split the variables for our hybrid operators.

Proposition 2.1. Let $(i, H, B)$ be an abstract Wiener space. In the following, the injection $i$ is implicitly understood, $H$ is identified with its dual space in such a way that (4) is verified and we fix a subspace $E$ in $\mathcal{F}\left(B^{\prime}\right)$. Let $E^{\perp}$ be the following space:

$$
E^{\perp}=\{x \in B, \quad u(x)=0 \quad \forall u \in E\} .
$$

Let $i_{1}$ denote the injection of $E^{\perp} \cap H$ in $E^{\perp}$. Then:

1. The system $\left(i_{1}, E^{\perp} \cap H, E^{\perp}\right)$ is an abstract Wiener space. We denote by $\mu_{E^{\perp}, h}$ the Gaussian measure of parameter $h$ on $E^{\perp}$.

2. We have, for all $h>0$ :

$$
\mu_{B, h}=\mu_{E, h} \otimes \mu_{E^{\perp}, h} .
$$

The map $x \rightarrow\left(P_{E}(x), x-P_{E}(x)\right)$ (where $P_{E}$ is defined in (6) ) is a bijection between $B$ and $E \times E^{\perp}$. We denote by $x=\left(x_{E}, x_{E^{\perp}}\right)$ the variable in $B$ and by $X=\left(X_{E}, X_{E^{\perp}}\right)$ the variable in $B^{2}$. Sometimes, we shall write $X=X_{E}+X_{E^{\perp}}$.

\subsection{Partial heat semigroup and hybrid operators}

If $E$ is in $\mathcal{F}\left(B^{\prime}\right)$, one can define, as in (22), on the one hand a heat semigroup acting only on the variables in $E$ and, on the other hand, another one acting on the variables of $E^{\perp}$ (defined in Proposition 2.1). There are two kinds of operators acting on the variables in $E$ : the first kind acts on a space of functions defined on $H$, the second kind, on a space of functions defined on $B$. For the operator acting on the variables of $E^{\perp}$, the second version only is available.

Let $E$ be in $\mathcal{F}\left(B^{\prime}\right)$. For all $t>0$, we define an operator $H_{E, t}$ on the space of bounded continuous functions on $H^{2}$, setting, when $F$ is such a function:

$$
\left(H_{E, t} F\right)(X)=\int_{E^{2}} F\left(X+Y_{E}\right) d \mu_{E^{2}, t}\left(Y_{E}\right) \quad X \in H^{2} .
$$

We define likewise an operator $\widetilde{H}_{E, t}$, defined by the same formula, but acting on the space of bounded Borel functions on $B^{2}$.

We may also define a partial heat semi-group, only acting on the variables lying in the subspace $E^{\perp}$. For all bounded Borel functions $F$ on $B^{2}$ and for all positive $t$, one may define a function $\widetilde{H}_{E^{\perp}, t} F$ on $B^{2}$ by setting, for all $X$ in $B^{2}$ :

$$
\left(\widetilde{H}_{E^{\perp}, t} F\right)(X)=\int_{\left(E^{\perp}\right)^{2}} F\left(X+Y_{E^{\perp}}\right) d \mu_{\left(E^{\perp}\right)^{2}, t}\left(Y_{E^{\perp}}\right) .
$$
6.

The operators $H_{E, t}$ and $\widetilde{H}_{E, t}$ will mainly appear in Section 3, The $\widetilde{H}_{E^{\perp}, t}$ will be used now and in Section

We are now ready to define the hybrid operator. 
Definition 2.2. If $F$ is a bounded Borel function on $B^{2}$ and if $E$ is in $\mathcal{F}\left(B^{\prime}\right)$, we denote by $Q_{h}^{\text {hyb,E}}(F)$ the quadratic form on $\mathcal{D} \times \mathcal{D}$ such that, for all $f$ and $g$ in $\mathcal{D}$, we have:

$$
Q_{h}^{h y b, E}(F)(f, g)=Q_{h}^{W e y l}\left(\widetilde{H}_{E^{\perp}, h / 2} F\right)(f, g) .
$$

The following result underlines the relationship between hybrid operators when associated with the same symbol, but with two different subspaces, one being included into the other one.

Let $F$ be a Borel function bounded on $B^{2}$. Let $E_{1}$ and $E_{2}$ be in $\mathcal{F}\left(B^{\prime}\right)$, such that $E_{1} \subset E_{2}$. Let $S$ be the orthogonal complement of $E_{1}$ in $E_{2}$ (for the scalar product of $H$ ). Then, we have:

$$
Q_{h}^{h y b, E_{1}}(F)=Q_{h}^{h y b, E_{2}}\left(\widetilde{H}_{S, h / 2} F\right)
$$

and we have:

$$
\widetilde{H}_{S, h / 2} F(X)=(\pi h)^{-\operatorname{dim}(S)} \int_{S^{2}} e^{\frac{|Y|^{2}}{h}} F(X+Y) d \lambda(Y) \quad X \in B^{2} .
$$

If $\left\{e_{1}, \ldots, e_{n}\right\}$ is an orthonormal basis of the orthogonal complement $S$ of $E_{1}$ in $E_{2}$, denoting by $D_{j}$ the subspace of $H$ spanned by $e_{j}$, we have:

$$
\widetilde{H}_{S, h / 2} F=\left[\prod_{j \leq n} \widetilde{H}_{D_{j}, h / 2}\right] F
$$

where $\widetilde{H}_{D_{j}, h / 2}$ is defined in (26).

The proof of (29) and (30) relies on the Definition 2.2:

$$
Q_{h}^{h y b, E_{j}}(F)=Q_{h}^{W e y l}\left(\widetilde{H}_{E_{j}^{\perp}, h / 2} F\right) \quad j=1,2
$$

and on the following equality:

$$
\widetilde{H}_{E_{1}^{\perp}, h / 2}=\widetilde{H}_{E_{2}^{\perp}, h / 2} \widetilde{H}_{S, h / 2}
$$

\section{Plan of the proof of Theorem 1.4}

Let $\left(e_{j}\right)_{(j \in \Gamma)}$ be a Hilbert basis of $H$, as in Definition 1.4. For every $j$ in $\Gamma$, we denote by $D_{j}$ the subspace of $H$ spanned by $e_{j}$. For every positive $t$, let $H_{D_{j}, t}$ and $\widetilde{H}_{D_{j}, t}$ be the operators defined in (26), the first one acting on functions on $H^{2}$, the second one, on functions on $B^{2}$. The integration domain in (26) is $D_{j}^{2}$, which is the subspace of $H^{2}$ spanned by $\left(e_{j}, 0\right)$ and $\left(0, e_{j}\right)$. For every finite subset $I$ of $\Gamma$, set:

$$
\widetilde{T}_{I, h}=\prod_{j \in I}\left(I-\widetilde{H}_{D_{j}, h / 2}\right), \quad \widetilde{S}_{I, h}=\prod_{j \in I} \widetilde{H}_{D_{j}, h / 2} .
$$

These operators act in the space of bounded Borel functions on $B^{2}$. We denote by $T_{I, h}$ the operator defined as in (32), but acting in the space of bounded continuous functions on $H^{2}$.

For every finite subset $I$ of $\Gamma$, let $E(I)$ be the subspace of $H$ spanned by the $e_{j}(j \in I)$.

$$
E(I)=\operatorname{Vect}\left\{\left(e_{j}\right), \quad j \in \Gamma\right\} .
$$

The main Theorem[1.4 is a consequence of the Propositions 3.1 et 3.3 below, which will be proved respectively in Sections 5 and 6 . 
Proposition 3.1. Let $F$ be in $S_{2}(M, \varepsilon)$. We assume that $F$ has a stochastic extension $\widetilde{F}$ for the measure $\mu_{B^{2}, h}$. For every finite subset $I$ in $\Gamma$, for all $h$ in $(0,1]$, there exists a bounded operator, which will be denoted by $O p_{h}^{h y b, E(I)}\left(\widetilde{T}_{I, h} \widetilde{F}\right)$ such that, for all $f$ and $g$ in $\mathcal{D}$, with the notations (28), (32) and (33):

$$
Q_{h}^{h y b, E(I)}\left(\widetilde{T}_{I, h} \widetilde{F}\right)(f, g)=<O p_{h}^{h y b, E(I)}\left(\widetilde{T}_{I, h} \widetilde{F}\right) f, g>.
$$

Moreover its norm satisfies:

$$
\left\|O p_{h}^{h y b, E(I)}\left(\widetilde{T}_{I, h} \widetilde{F}\right)\right\|_{\mathcal{L}\left(L^{2}\left(B, \mu_{B, h / 2}\right)\right)} \leq M\left(81 \pi h S_{\varepsilon}\right)^{|I|} \prod_{j \in I} \varepsilon_{j}^{2} .
$$

If one admits Proposition 3.1 (which is a consequence of the combined Propositions 5.2 and 5.3), one can prove the following result.

Proposition 3.2. Let $F$ be a function in $S_{2}(M, \varepsilon)$, where the family $\left(\varepsilon_{j}\right)_{(j \in \Gamma)}$ is square summable. Set $h>0$. We assume that $F$ has a stochastic extension $\widetilde{F}$ for the measure $\mu_{B^{2}, h}$. Then, for every increasing sequence $\left(\Lambda_{n}\right)$ of finite subsets in $\Gamma$, whose union is $\Gamma$, there exists a sequence of operators, denoted by $\left(O p_{h}^{h y b, E\left(\Lambda_{n}\right)}(\widetilde{F})\right)$, such that, with the notations (28) and (39), for all $f, g$ in $\mathcal{D}$,:

$$
Q_{h}^{h y b, E\left(\Lambda_{n}\right)}(\widetilde{F})(f, g)=<\left(O p_{h}^{h y b, E\left(\Lambda_{n}\right)}(\widetilde{F})\right) f, g>
$$

Moreover, the sequence of operators $\left(O p_{h}^{h y b, E\left(\Lambda_{n}\right)}(\widetilde{F})\right)_{(n \geq 1)}$ is a Cauchy sequence in $\mathcal{L}\left(L^{2}\left(B, \mu_{B, h / 2}\right)\right)$. Its limit, denoted by $O p_{h}^{W e y l}(F)$, satisfies (18).

Proof. We have, for every continuous and bounded function $G$ on $B^{2}$, for any finite subset $\Lambda$ in $\Gamma$ :

$$
G=\sum_{I \subseteq \Lambda} \widetilde{T}_{I, h} \widetilde{S}_{\Lambda \backslash I, h} G
$$

The sum runs over all the subsets in $\Lambda$, including the empty set and $\Lambda$ itself. As a consequence, the equality (36) will be satisfied if we set:

$$
Q_{h}^{h y b, E(\Lambda)}(\widetilde{F})=\sum_{I \subseteq \Lambda} Q_{h}^{h y b, E(\Lambda)}\left(\widetilde{T}_{I, h} \widetilde{S}_{\Lambda \backslash I, h} \widetilde{F}\right) .
$$

From (29) and (30), applied with subspaces $E(I)$ and $E(\Lambda)$ :

$$
Q_{h}^{h y b, E(\Lambda)}(\widetilde{F})=\sum_{I \subseteq \Lambda} Q_{h}^{h y b, E(I)}\left(\widetilde{T}_{I, h} \widetilde{F}\right) .
$$

The term corresponding to $I=\emptyset$ is the anti-Wick quadratic form associated with $\widetilde{F}$ and adapted to the Gaussian measure. Therefore, according to Proposition 3.1. there exists a bounded operator, denoted by $O p_{h}^{h y b, E(\Lambda)}$ such that 36 is satisfied. If $\left(\Lambda_{n}\right)$ is an increasing sequence of finite subsets of $\Gamma$, we then have, if $m<n$ :

$$
O p_{h}^{h y b, E\left(\Lambda_{n}\right)}(\widetilde{F})-O p_{h}^{h y b, E\left(\Lambda_{m}\right)}(\widetilde{F})=\sum_{I \in \mathcal{P}(m, n)} O p_{h}^{h y b, E(I)}\left(\widetilde{T}_{I, h} \widetilde{F}\right)
$$

where $\mathcal{P}(m, n)$ is the family of subsets $I$ in $\Gamma$, included in $\Lambda_{n}$, but with at least one element not belonging to $\Lambda_{m}$. From (40) and from Proposition 3.1, we have, when $m<n$ :

$$
\begin{aligned}
\left\|O p_{h}^{h y b, E\left(\Lambda_{n}\right)}(\widetilde{F})-O p_{h}^{h y b, E\left(\Lambda_{m}\right)}(\widetilde{F})\right\|_{\mathcal{L}\left(L^{2}\left(B, \mu_{B, h / 2}\right)\right)} & \leq \sum_{I \in \mathcal{P}(m, n)}\left\|O p_{h}^{h y b, E(I)}\left(\widetilde{T}_{I, h} \widetilde{F}\right)\right\|_{\mathcal{L}\left(L^{2}\left(B, \mu_{B, h / 2}\right)\right)} \\
& \leq M \sum_{I \in \mathcal{P}(m, n)}\left(81 \pi h S_{\varepsilon}\right)^{|I|} \prod_{j \in I} \varepsilon_{j}^{2} .
\end{aligned}
$$


As a consequence, if $m<n$ :

$$
\left\|O p_{h}^{h y b, E\left(\Lambda_{n}\right)}(\widetilde{F})-O p_{h}^{h y b, E\left(\Lambda_{m}\right)}(\widetilde{F})\right\|_{\mathcal{L}\left(L^{2}\left(B, \mu_{B, h / 2}\right)\right)} \leq M 81 \pi h S_{\varepsilon}\left[\sum_{j \in \Lambda_{n} \backslash \Lambda_{m}} \varepsilon_{j}^{2}\right] \prod_{k \in \Lambda_{n}}\left(1+81 \pi h S_{\varepsilon} \varepsilon_{k}^{2}\right) .
$$

If the family $\left(\varepsilon_{j}^{2}\right)_{(j \in \Gamma)}$ is summable, the above right hand-side product stays bounded independently of $n$, whereas the sum tends to 0 when $m \rightarrow+\infty$. As a consequence, the sequence $\left(O p_{h}^{h y b, E\left(\Lambda_{n}\right)}(\widetilde{F})\right)$ is a Cauchy sequence in $\mathcal{L}\left(L^{2}\left(B, \mu_{B, h / 2}\right)\right)$. Likewise:

$$
\left\|O p_{h}^{h y b, E\left(\Lambda_{n}\right)}(\widetilde{F})\right\|_{\mathcal{L}\left(L^{2}\left(B, \mu_{B, h / 2}\right)\right)} \leq M \prod_{k \in \Lambda_{n}}\left(1+81 \pi h S_{\varepsilon} \varepsilon_{k}^{2}\right) .
$$

Therefore, the limit of this sequence of operators, denoted by $O p_{h}^{W e y l}(F)$, verifies (18).

One could think that the operator $O p_{h}^{W e y l}(F)$ depends on the sequence $\left(\Lambda_{n}\right)$, but the following proposition proves that it is not the case. It will be proved in Section 6 .

Proposition 3.3. Let $F$ belong to $S_{2}(M, \varepsilon)$, where the sequence $\left(\varepsilon_{j}\right)_{(j \in \Gamma)}$ is square summable. Set $h>0$. Define the function $\widetilde{F}$ on $B^{2}$ as the stochastic extension of $F$ both for the measure $\mu_{B^{2}, h}$ and for the measure $\mu_{B^{2}, h / 2}$. Let $\left(\Lambda_{n}\right)$ be an increasing sequence of finite subsets of $\Gamma$, whose union is $\Gamma$. Then, we have, for every $f$ and $g$ in $\mathcal{D}$, setting $E_{n}=E\left(\Lambda_{n}\right)$

$$
\lim _{n \rightarrow+\infty} Q_{h}^{W e y l}\left(\widetilde{H}_{E_{n}^{\perp}, h / 2} \widetilde{F}\right)(f, g)=Q_{h}^{W e y l}(\widetilde{F})(f, g)
$$

One can notice that, for a given function $F$ in $S_{2}(M, \varepsilon)$, the stochastic extension (for the measure $\mu_{B^{2}, s}$ ) $\widetilde{F}$ is unique, but only up to a $\mu_{B^{2}, s^{-}}$negligible set. Nevertheless, if $\widetilde{F}$ and $\widetilde{G}$ are two stochastic extensions of the same function $F$, for the measures $\mu_{B^{2}, h}$ and $\mu_{B^{2}, h / 2}$, one can check that $\widetilde{H}_{E_{n}^{\perp}, h / 2} \widetilde{F}$ and $\widetilde{H}_{E_{n}^{\perp}, h / 2} \widetilde{G}$ are equal almost everywhere for the measure $\mu_{B^{2}, h / 2}$.

End of the proof of Theorem 1.4. Once Proposition 3.2 has been established, it only remains to prove (17). Now for all $f$ and $g$ in $\mathcal{D}$ one has, setting $E_{n}=E\left(\Lambda_{n}\right)$ :

$$
<O p_{h}^{W e y l}(F) f, g>=\lim _{n \rightarrow+\infty}<\left(O p_{h}^{h y b, E_{n}}(\widetilde{F})\right) f, g>=\lim _{n \rightarrow+\infty} Q_{h}^{W e y l}\left(\widetilde{H}_{E_{n}^{\perp}, h / 2} \widetilde{F}\right)(f, g) .
$$

Hence the equality (17) of Theorem 1.4 comes from the above equality and from (41).

\section{Some useful operators in Wiener spaces}

We first recall the precise definition of Wiener spaces as well as some of their properties. Then we adapt some classical notions for Wiener spaces : coherent states, Segal-Bargmann transformation. Next we give properties of the Gaussian Wigner function of Section 1, This will allow us to write Definition 1.2 of the Weyl operators and Definition 2.2 of the hybrid operators in a way more suitable for norm estimates. This will yield Proposition 4.11. which will be used in Section 5 to prove Proposition 3.1.

\subsection{Abstract Wiener spaces}

If $H$ is a real separable infinite-dimensional Hilbert space, it is impossible to define on its Borel $\sigma$-algebra a measure $\mu_{H, h}$ such that (7) holds with $B$ instead of $H$.

Nevertheless, one can define a promeasure (or cylindrical probability measure in the sense of [K-R] $\mu_{H, h}$ on the cylinder sets of $H$. For every $E$ in $\mathcal{F}(H)$ and every positive $h$, one can define a Gaussian measure $\mu_{E, h}$ 
on $E$ by (5), where $\lambda_{E}$ is the Lebesgue measure on $E$ (normalized in a natural way) and $|\cdot|$ is the norm of $H$. A cylinder set of $H$ is any set of the form $C=\pi_{E}^{-1}(\Omega)$, where $E \in \mathcal{F}(H), \pi_{E}: H \rightarrow E$ is the orthogonal projection and $\Omega$ is a Borel set of $E$. If $C$ is such a cylinder set, one sets $\mu_{H, h}(C)=\mu_{E, h}(\Omega)$. In other words, for every Borel set $\Omega$ of $E$ :

$$
\mu_{H, h}\left(\pi_{E}^{-1}(\Omega)\right)=(2 \pi h)^{-\operatorname{dim}(E) / 2} \int_{\Omega} e^{-\frac{|y|^{2}}{2 h}} d \lambda(y) .
$$

One defines this way an additive set function on the cylinder sets of $H$, but if $H$ is infinite-dimensional, this function is not $\sigma$-additive and $\mu_{H, h}$ does not extend as a measure on the $\sigma$-algebra generated by the cylinder sets (which is the Borel $\sigma$-algebra).

If the Hilbert space $H$ is included into a Banach space $H$, the canonical injection being continuous and having a dense range, so that (4) is satisfied, one can define the cylinder sets of $B$ as the sets $C=P_{E}^{-1}(\Omega)$, where $E \in \mathcal{F}\left(B^{\prime}\right)$, where $P_{E}: B \rightarrow E$ is defined by (6) and where $\Omega$ is a Borel set of $E$. One then defines an additive set function $\mu_{B, h}$ on the cylinder sets of $B$ as in (42):

$$
\mu_{B, h}\left(P_{E}^{-1}(\Omega)\right)=(2 \pi h)^{-\operatorname{dim}(E) / 2} \int_{\Omega} e^{-\frac{|y|^{2}}{2 h}} d \lambda(y) .
$$

But this time, if $B$ is well chosen, the additive sets function $\mu_{B, h}$ extends as a measure on the Borel $\sigma$-algebra of $B$. The following definition specifies the conditions which $B$ must fulfill.

Definition 4.1. [G-2, $G-3,[K]$ An abstract Wiener space is a triple $(i, H, B)$ where $H$ is a real separable Hilbert space, $B$ a Banach space and $i$ a continuous injection from $H$ into $B$, such that $i(H)$ is dense in $B$ and satisfying, moreover, the following condition. For all positive $\varepsilon$ and $h$, there exists a subspace $F$ in $\mathcal{F}(H)$ such that, for all $E$ in $\mathcal{F}(H)$, orthogonal to $F$,

$$
\mu_{H, h}\left(\left\{x \in H, \quad\left\|i\left(\pi_{E}(x)\right)\right\|_{B}>\varepsilon\right\}\right)<\varepsilon .
$$

The norm on $B$ is said to be "measurable".

If $(i, H, B)$ is an abstract Wiener space (in other words if the norm of $B$ is measurable in the sense above) and if (4) holds one proves that, for every positive $h$, the additive set function $\mu_{B, h}$, defined on the cylinder set functions of $B$, extends as a measure on the Borel $\sigma$-algebra of $B$ and has the following property. For every finite system $\left\{u_{1}, \ldots, u_{n}\right\}$ of $B^{\prime}$, which is orthonormal with respect to the scalar product of $H$, the functions $x \rightarrow u_{j}(x)$ (defined on $B$ ) are independent Gaussian random variables and the system $\left\{u_{1}, \ldots u_{n}\right\}$ has the normal distribution $\mu_{\mathbb{R}^{n}, h}$. See $\mathrm{G}-2$, $\mathrm{G}-3$ and $[\mathrm{K}$ (consequence of the Theorems 4.1 and 4.2, Chapter 1). For every $E$ in $\mathcal{F}\left(B^{\prime}\right)$ and every $\varphi$ in $L^{1}\left(E, \mu_{E, h}\right)$, the equality (77) is satisfied, according to the transfer Theorem.

Let us recall a classical example of the explicit computation of an integral, where $a$ is in the complexified space $H_{\mathbf{C}}$ :

$$
\int_{B} e^{\ell_{a}(x)} d \mu_{B, h}(x)=e^{h \frac{a^{2}}{2}} .
$$

One has set $a^{2}=|u|^{2}-|v|^{2}+2 i u \cdot v$ if $a=u+i v$, with $u$ and $v$ in $H$. Let us recall, too, that for all $a$ in $H$ and for all $p \geq 1$ :

$$
\int_{B}\left|\ell_{a}(x)\right|^{p} d \mu_{B, h}(x)=\frac{(2 h)^{p / 2}}{\sqrt{\pi}}|a|^{p} \Gamma\left(\frac{p+1}{2}\right) .
$$

One sees, too, that for all $a$ and $B$ in $H$,

$$
\int_{B} e^{\ell_{b}(u)}\left|\ell_{a}(u)\right|^{p} d \mu_{B, h}(u)=e^{h \frac{|b|^{2}}{2}} \int_{\mathbb{R}}|\sqrt{h}| a|v+h a \cdot b|^{p} d \mu_{\mathbb{R}, 1}(v) .
$$

The following proposition allows to deal with translations by a vector $a$ belonging to $H$. There is no such result for a translation by a vector $a$ belonging to $B$. 
Proposition 4.2. $[K], p$ 113,114 Let $(i, H, B)$ be an abstract Wiener space and $\mu_{B, h}$ its measure. For all $g \in L^{1}\left(B, \mu_{B, h}\right)$, one has, for all a in $H$ :

$$
\int_{B} g(x) d \mu_{B, h}(x)=e^{-\frac{1}{2 h}|a|^{2}} \int_{B} g(x+a) e^{-\frac{1}{h} \ell_{a}(x)} d \mu_{B, h}(x) .
$$

For every $a \in H$ and every $f$ in $L^{2}\left(B, \mu_{B, h}\right)$, one has

$$
\int_{B}|f(x)|^{2} d \mu_{B, h}(x)=e^{-\frac{1}{2 h}|a|^{2}} \int_{B}|f(x+a)|^{2} e^{-\frac{1}{h} \ell_{a}(x)} d \mu_{B, h}(x) .
$$

Let us recall the theorem of Wick :

Theorem 4.3. Wick Let $u_{1}, \ldots u_{2 p}$ be vectors of $H(p \geq 1)$. Let $h>0$. Then one has

$$
\int_{B} \ell_{u_{1}}(x) \ldots \ell_{u_{2 p}}(x) d \mu_{B, h}(x)=h^{p} \sum_{(\varphi, \psi) \in S_{p}} \prod_{j=1}^{p}<u_{\varphi(j)}, u_{\psi(j)}>
$$

where $S_{p}$ is the set of all couples $(\varphi, \psi)$ of injections from $\{1, \ldots, p\}$ into $\{1, \ldots, 2 p\}$ such that:

1. For all $j \leq p, \varphi(j)<\psi(j)$.

2. The sequence $(\varphi(j))_{(1 \leq j \leq k)}$ is an increasing sequence.

One deduces from (43) the following inequalities, which hold for all $a$ and $b$ in the complexified of $H$ :

$$
\begin{aligned}
& \int_{B}\left|e^{\ell_{a}(x)}-e^{\ell_{b}(x)}\right|^{2} d \mu_{B, h}(x) \leq 4 h|a-b|(|a|+|b|) e^{2 h \max \left(|\operatorname{Re} a|^{2},|\operatorname{Re} b|^{2}\right)} \\
& \int_{B}\left|e^{\ell_{a}(x)}-e^{\ell_{b}(x)}\right|^{2} d \mu_{B, h}(x) \leq e^{2 h \max (|\operatorname{Re}(a)|,|\operatorname{Re}(b)|)^{2}} h|a-b|^{2}\left(1+4 h \max (|\operatorname{Re}(a)|,|\operatorname{Re}(b)|)^{2}\right)
\end{aligned}
$$

\subsection{Stochastic extensions}

In order to define the stochastic extension of a function $f: H \rightarrow \mathbf{C}$, we first define the extension of the orthogonal projection $\pi_{E}: H \rightarrow E$, where $E \in \mathcal{F}(H)$.

With each $E$ in $\mathcal{F}(H)$, one can associate a map $\widetilde{\pi}_{E}: B \rightarrow E$ defined almost everywhere by

$$
\widetilde{\pi}_{E}(x)=\sum_{j=1}^{\operatorname{dim}(E)} \ell_{u_{j}}(x) u_{j}
$$

where the $u_{j}(1 \leq j \leq \operatorname{dim}(E))$ form an orthonormal basis of $E$ and $\ell_{u_{j}}$ is defined in Proposition 1.1. This map does not depend on the choice of the orthonormal basis and, when $E \subset B^{\prime}$, it coincides with the map $P_{E}$ already defined by (6).

Remark that (7) still holds for the subspaces $E$ in $\mathcal{F}(H)$ (and not necessarily in $\mathcal{F}\left(B^{\prime}\right)$ ), provided $P_{E}$ is replaced by $\widetilde{\pi}_{E}$. See Lemma 4.7 in $[\mathrm{K}]$.

Notice that, for every subspace $E$ in $\mathcal{F}(H)$ :

$$
a \cdot\left(\widetilde{\pi}_{E}(x)\right)=\ell_{\pi_{E}(a)}(x) \quad a \in H \quad \text { a.e. } x \in B
$$

The following notion has been introduced by Gross [G-1], who gives conditions for the existence of the extension. Other conditions or examples will be found in Section 8 or in A-J-N-1. 
Definition 4.4. [G-1, G-2, G-3] [RA] [K] Let $(i, H, B)$ be an abstract Wiener space satisfying [4). (The inclusion $i$ will be omitted). Let $h$ be a positive real number.

1. A Borel function $f$, defined on $H$, is said to admit a stochastic extension $\widetilde{f}$ with respect to the measure $\mu_{B, h}$ if, for every increasing sequence $\left(E_{n}\right)$ in $\mathcal{F}(H)$, whose union is dense in $H$, the sequence of functions $f \circ \widetilde{\pi}_{E_{n}}$ (where $\widetilde{\pi}_{E_{n}}$ is defined by (50)) converges in probability with respect to the measure $\mu_{B, h}$ to $\widetilde{f}$. In other words, if, for every $\delta>0$,

$$
\lim _{n \rightarrow+\infty} \mu_{B, h}\left(\left\{x \in B, \quad\left|f \circ \widetilde{\pi}_{E_{n}}(x)-\widetilde{f}(x)\right|>\delta\right\}\right)=0 .
$$

2. A function $f$ is said to admit a stochastic extension $\tilde{f} \in L^{p}\left(B, \mu_{B, h}\right)$ in the sense of $L^{p}\left(B, \mu_{B, h}\right)(1 \leq p<$ $\infty)$ if, for every increasing sequence $\left(E_{n}\right)$ in $\mathcal{F}(H)$, whose union is dense in $H$, the functions $f \circ \widetilde{\pi}_{E_{n}}$ are in $L^{p}\left(B, \mu_{B, h}\right)$ and if the sequence $f \circ \widetilde{\pi}_{E_{n}}$ converges in $L^{p}\left(B, \mu_{B, h}\right)$ to $\widetilde{f}$.

One defines likewise the stochastic extension of a function on $H^{2}$ to a function on $B^{2}$.

If $\tilde{f}$ is the stochastic extension of a function $f: H \rightarrow H$, one cannot say that $f$ is the restriction of $\tilde{f}$ to $H$. Since $H$ is negligible (see Kuo $[\overline{\mathrm{K}}$ ), this is irrelevant. For every $a$ in $H$ one sees that the application $u \rightarrow u \cdot a$, defined on $H$, admits a stochastic extension which is the function $\ell_{a}$. This is a consequence of the equality (51). In Definition 4.4, the functions can take their values in a Banach space. Hence one can say that the application $\widetilde{\pi}_{E}$ of (50) is the stochastic extension of the orthogonal projection $\pi_{E}: H \rightarrow E$. One will find in Section 8 examples of functions admitting stochastic extensions. In particular, if a function $f$ is bounded on $H$ and uniformly continuous with respect to the restriction to $H$ of the norm of $B$, then it admits a stochastic extension $\widetilde{f}$, which coincides with its density extension (see Kuo $[\underline{\mathrm{K}}]$, Chapter 1, Theorem 6.3).

If a Borel function $f$ is bounded on $H$, does not depend on $h$ and admits, for every positive $h$, a stochastic extension with respect to $\mu_{B, h}$, this extension may depend on $h$. It is not the case in the situation of Proposition 8.2. In the other cases, we may consider that the stochastic extension is independent of $h$ if $h$ varies in a countable subset $Q$ of $(0,+\infty)$.

\subsection{The Heat operator (continued)}

In the rest of this work, $(i, H, B)$ represents an abstract Wiener space satisfying (4) and the injection $i$ will be omitted. We complete the investigation begun in Sections 2.1 and 2.3 .

Let $E$ be in $\mathcal{F}\left(B^{\prime}\right)$, let $E^{\perp}$ be its orthogonal space, defined in (24) and let $t>0$. For every Borel function $F$ bounded on $B^{2}$, let $\widetilde{H}_{E^{\perp}, t} F$ be the function defined in (27):

$$
\left(\widetilde{H}_{E^{\perp}, t} F\right)(X)=\int_{\left(E^{\perp}\right)^{2}} F(X+Y) d \mu_{\left(E^{\perp}\right)^{2}, t}(Y) .
$$

For all real numbers $h_{1}>0$ and $h_{2}>0$, one defines a probability measure on $B^{2}$, using Proposition 2.1, by:

$$
\nu_{B^{2}, E^{2}, h_{1}, h_{2}}=\mu_{E^{2}, h_{1}} \otimes \mu_{\left(E^{\perp}\right)^{2}, h_{2}} .
$$

Proposition 4.5. Let $h_{1}, h_{2}$ and $t$ be positive real numbers. The operator $\widetilde{H}_{E^{\perp}, t}$ is bounded and its norm is at most 1 from $L^{p}\left(B^{2}, \nu_{B^{2}, E^{2}, h_{1}, h_{2}+t}\right)$ into $L^{p}\left(B^{2}, \nu_{B^{2}, E^{2}, h_{1}, h_{2}}\right)$ for every $p \in[1,+\infty]$. Let

$$
\left(\widetilde{M}_{E^{\perp}, t, h_{1}, h_{2}} G\right)(X)=\int_{\left(E^{\perp}\right)^{2}} G\left(X_{E}, Y_{E^{\perp}}+\frac{h_{2}}{t+h_{2}} X_{E^{\perp}}\right) d \mu_{\left(E^{\perp}\right)^{2}, \frac{t h_{2}}{t+h_{2}}}\left(Y_{E^{\perp}}\right) .
$$

The operator $\widetilde{M}_{E^{\perp}, t, h_{1}, h_{2}}$ is bounded and its norm is at most 1 from $L^{p}\left(B^{2}, \nu_{B^{2}, E^{2}, h_{1}, h_{2}}\right)$ into $L^{p}\left(B^{2}, \nu_{B^{2}, E^{2}, h_{1}, h_{2}+t}\right)$ for every $p \in[1,+\infty]$. Moreover, for all functions $F \in L^{p}\left(B^{2}, \nu_{B^{2}, E^{2}, h_{1}, h_{2}+t}\right)$ and $G \in L^{q}\left(B^{2}, \nu_{B^{2}, E^{2}, h_{1}, h_{2}}\right)$, with $p$ and $q$ conjugate exponents, one has:

$$
\int_{B^{2}} \widetilde{H}_{E^{\perp}, t} F(X) G(X) d \nu_{B^{2}, E^{2}, h_{1}, h_{2}}(X)=\int_{B^{2}} F(X) \widetilde{M}_{E^{\perp}, t, h_{1}, h_{2}} G(X) d \nu_{B^{2}, E^{2}, h_{1}, h_{2}+t}(X) .
$$


Proof. Suppose first that $p$ is finite. Let $F$ be a bounded cylindrical Borel function : there exist a subspace $D$ in $\mathcal{F}\left(B^{\prime}\right)$ and a Borel function $\widehat{F}$, bounded on $D^{2}$, such that, for every $X \in B^{2}, F(X)=\widehat{F}\left(P_{D}(X)\right)$. One can assume, without loss of generality, that $E \subset D$. Let $\left(e_{1}, \ldots, e_{s}, e_{s+1}, \ldots, e_{d}\right)$ be a basis of $D$, orthonormal with respect to the scalar product of $H,\left(e_{1}, \ldots, e_{s}\right)$ being a basis of $E$. Let us use the following notation :

$$
\widehat{F}\left(\sum_{k=1}^{d} x_{k} e_{k}, \sum_{k=1}^{d} \xi_{k} e_{k}\right)=f\left(\widetilde{x}, x^{\prime}, \widetilde{\xi}, \xi^{\prime}\right)
$$

with $\widetilde{x}=\left(x_{1}, \ldots, x_{s}\right), x^{\prime}=\left(x_{s+1}, \ldots, x_{d}\right)$ and the same convention for $\xi$. Let $p \in[1,+\infty[$. According to Jensen's inequality,

$$
\left\|\widetilde{H}_{E^{\perp}, t} F\right\|_{L^{p}\left(B^{2}, \nu_{B^{2}, E^{2}, h_{1}, h_{2}}\right)}^{p} \leq \int_{B^{2}} \int_{\left(E^{\perp}\right)^{2}}\left|\widehat{F}\left(P_{D}(X+Y)\right)\right|^{p} d \mu_{\left(E^{\perp}\right)^{2}, t}(Y) d \mu_{E^{2}, h_{1}}\left(X_{E}\right) d \mu_{\left(E^{\perp}\right)^{2}, h_{2}}\left(X_{E^{\perp}}\right),
$$

therefore, by (7),

$$
\begin{aligned}
& \left\|\widetilde{H}_{E^{\perp}, t} F\right\|_{L^{p}\left(B^{2}, \nu_{B^{2}, E^{2}, h_{1}, h_{2}}\right)}^{p} \leq(2 \pi t)^{-d+s}\left(2 \pi h_{1}\right)^{-s}\left(2 \pi h_{2}\right)^{-d+s} \\
& \int_{\mathbb{R}^{4 d-2 s}}\left|f\left(\widetilde{x}, x^{\prime}+y^{\prime}, \widetilde{\xi}, \xi^{\prime}+\eta^{\prime}\right)\right|^{p} e^{-\frac{1}{2 t}\left(\left|y^{\prime}\right|^{2}+\left|\eta^{\prime}\right|^{2}\right)} e^{-\frac{1}{2 h_{1}}\left(|\widetilde{x}|^{2}+|\widetilde{\xi}|^{2}\right)} e^{-\frac{1}{2 h_{2}}\left(\left|x^{\prime}\right|^{2}+\left|\xi^{\prime}\right|^{2}\right)} d \lambda\left(\widetilde{x}, x^{\prime}, y^{\prime}, \widetilde{\xi}, \xi^{\prime}, \eta^{\prime}\right) .
\end{aligned}
$$

The transition from $x^{\prime}+y^{\prime}, \xi^{\prime}+\eta^{\prime}$ to $z^{\prime}, \zeta^{\prime}$ and a classical computation on the convolution of Gaussian functions give

$$
\begin{aligned}
& \left\|\widetilde{H}_{E^{\perp}, t} F\right\|_{L^{p}\left(B^{2}, \nu_{B^{2}, E^{2}, h_{1}, h_{2}}\right)}^{p} \\
& \leq\left(2 \pi h_{1}\right)^{-s}\left(2 \pi\left(h_{2}+t\right)\right)^{s-d} \int_{\mathbb{R}^{2 d}}|f(z, \zeta)|^{p} e^{-\frac{1}{2\left(h_{2}+t\right)}\left(\left|z^{\prime}\right|^{2}+\left|\zeta^{\prime}\right|^{2}\right)} e^{-\frac{1}{2 h_{1}}\left(|\widetilde{z}|^{2}+|\widetilde{\zeta}|^{2}\right)} d \lambda\left(\widetilde{z}, z^{\prime}, \widetilde{\zeta}, \zeta^{\prime}\right) \\
& =\int_{B^{2}}|F(X)|^{p} d \mu_{E^{2}, h_{1}}\left(X_{E}\right) d \mu_{\left(E^{\perp}\right)^{2}, h_{2}+t}\left(X_{E^{\perp}}\right) .
\end{aligned}
$$

One extends this result, using the density of the cylindrical functions in $L^{p}\left(B^{2}, \nu_{B^{2}, E^{2}, h_{1}, h_{2}+t}\right)$.

If $F \in L^{\infty}\left(B^{2}, \nu_{B^{2}, E^{2}, h_{1}, h_{2}+t}\right)$, let $G$ be the function equal to $F$ on the set

$$
\left\{X \in B^{2}:|F(X)| \leq\|F\|_{L^{\infty}\left(B^{2}, \nu_{B^{2}, E^{2}, h_{1}, h_{2}+t}\right)}\right\}
$$

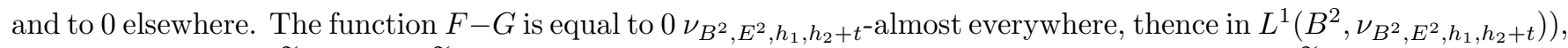
which implies that $\widetilde{H}_{E^{\perp}, t} F=\widetilde{H}_{E^{\perp}, t} G \nu_{B^{2}, E^{2}, h_{1}, h_{2}}$ almost everywhere. Now, for all $X$ in $B^{2}, \widetilde{H}_{E^{\perp}, t} G(X) \leq$ $\|F\|_{L^{\infty}\left(B, \nu_{B^{2}, E^{2}, h_{1}, h_{2}+t}\right)}$, then $\widetilde{H}_{E^{\perp}, t} F(X) \leq\|F\|_{L^{\infty}\left(B, \nu_{B^{2}, E^{2}, h_{1}, h_{2}+t}\right)}$ for $\nu_{B^{2}, E^{2}, h_{1}, h_{2}}$ - almost every $X$.

The result about $\widetilde{M}_{E^{\perp}, t, h_{1}, h_{2}}$ can be proved in a similar way. One can also introduce the partial dilation $\Delta_{E^{\perp}, \lambda}$, defined by

$$
\Delta_{E^{\perp}, \lambda} G\left(X_{E}, X_{E^{\perp}}\right)=G\left(X_{E}, \lambda X_{E^{\perp}}\right),
$$

which is isometrical from $L^{p}\left(B^{2}, \nu_{B^{2}, E^{2}, h_{1}, h_{2}}\right)$ in $L^{p}\left(B^{2}, \nu_{B^{2}, E^{2}, h_{1}, h_{2} / \lambda^{2}}\right)$ and remark that

$$
\widetilde{M}_{E^{\perp}, t, h_{1}, h_{2}}=\Delta_{E^{\perp}, \frac{h_{2}}{t+h_{2}}} \circ \widetilde{H}_{E^{\perp}, \frac{t h_{2}}{t+h_{2}}} \cdot
$$

The equality (55) is the result of computations similar to the precedent ones. $B^{2}$.

When $E=\left\{0_{B}\right\}$, this yields results for the global heat operator, the product measures being "global" on $L^{p}$.

Gross and Kuo gave results about these operators in the case when they act on functions more regular than 


\subsection{Coherent states and Segal Bargmann transformation}

For every $E$ in $\mathcal{F}(H)$, the coherent states on $E$ are functions $\Psi_{X, h}^{E, L e b}$ defined, for all $X=(a, b) \in E^{2}$ and for any $h>0$, by:

$$
\Psi_{X, h}^{E, L e b}(u)=(\pi h)^{-\operatorname{dim}(E) / 4} e^{-\frac{|u-a|^{2}}{2 h}} e^{\frac{i}{h} u \cdot b-\frac{i}{2 h} a . b} \quad X=(a, b) \in E^{2} \quad u \in E .
$$

It is well known that, for all $u$ and $v$ in $L^{2}(E, \lambda)$, one has

$$
<u, v>_{L e b}=(2 \pi h)^{-\operatorname{dim}(E)} \int_{E^{2}}<u, \Psi_{X, h}^{E, L e b}>_{L e b}<\Psi_{X, h}^{E, L e b}, v>_{L e b} d \lambda(X)
$$

where $\langle\cdot, \cdot\rangle_{L e b}$ is the scalar product of $L^{2}(E, \lambda)$.

One can also define Gaussian coherent states, which are functions on the Hilbert space $H$. For every $X=(a, b)$ in $H^{2}$, one defines a function $\Psi_{X, h}$ on $H$, setting:

$$
\Psi_{X, h}(u)=e^{\frac{1}{h} u \cdot(a+i b)-\frac{1}{2 h}|a|^{2}-\frac{i}{2 \hbar} a \cdot b} .
$$

This function is only defined for $u$ in $H$. It cannot be used as it is in any integral, but it admits a stochastic extension in the sense of $L^{2}\left(B, \mu_{B, h / 2}\right)$ (see Definition 4.4). This extension is defined by

$$
\widetilde{\Psi}_{X, h}(u)=e^{\frac{1}{h} \ell_{a+i b}(u)-\frac{1}{2 h}|a|^{2}-\frac{i}{2 h} a \cdot b} \quad X=(a, b) \in H^{2} \text { a.e. } u \in B .
$$

The norm of the extension is equal to 1 (see (44)). According to (43), one has, for all $U$ and $V$ in $H$

$$
<\widetilde{\Psi}_{U, h}, \widetilde{\Psi}_{V, h}>_{L^{2}\left(B, \mu_{B, h / 2}\right)}=e^{-\frac{1}{4 h}|U-V|^{2}+\frac{i}{2 h} \sigma(U, V)} .
$$

For every $E$ in $\mathcal{F}(H)$ and every $X$ in $E^{2}$ one can write:

$$
\Psi_{X, h}=\left(\gamma_{E, h / 2}^{-1} \Psi_{X, h}^{E, L e b}\right) \circ P_{E}
$$

To define the Segal-Bargmann transformation one starts from its well-known analog in a finite dimensional case (see $[\mathrm{F}$ or $[\mathrm{SJ}])$ ). For every $E$ in $\mathcal{F}(H)$ and every function $\varphi$ in $\mathcal{S}_{E}$, one defines a function $\widehat{T}_{h} \varphi$ on $E^{2}$ setting:

$$
\left(\widehat{T}_{h} \varphi\right)(x, \xi)=e^{-\frac{1}{4 h}(x-i \xi)^{2}} \int_{E} \varphi(y) e^{\frac{1}{h} y \cdot(x-i \xi)} d \mu_{E, h / 2}(y) \quad(x, \xi) \in E^{2}
$$

where $(x-i \xi)^{2}=|x|^{2}-|\xi|^{2}-2 i x \cdot \xi$. One knows $([\mathrm{F}])$ that $\widehat{T}_{h}$ is a partial isometry from $L^{2}\left(E, \mu_{E h / 2}\right)$ in $L^{2}\left(E^{2}, \mu_{E^{2}, h}\right)$ and that, for all $Z=(z, \zeta)$ in $E^{2}$ :

$$
\left(\widehat{T}_{h} \varphi\right)(z, \zeta)=\int_{E^{2}} e^{\frac{1}{2 h}(x+i \xi) \cdot(z-i \zeta)}\left(\widehat{T}_{h} \varphi\right)(x, \xi) d \mu_{E^{2}, h}(x, \xi) .
$$

One can see, too, that:

$$
\widehat{T}_{h} \varphi(X)=e^{\frac{1}{4 h}|X|^{2}}<\gamma_{E, h / 2} \varphi, \Psi_{X, h}^{E, L e b}>_{L e b}
$$

For every function $f$ in $\mathcal{D}$, of the form $f=\widehat{f} \circ P_{E}$, with $E$ in $\mathcal{F}\left(B^{\prime}\right)$ and $\widehat{f}$ in $\mathcal{S}_{E}$, one defines a function $T_{h} f$ on $B^{2}$ by:

$$
\left(T_{h} f\right)(X)=\left(\widehat{T}_{h} \widehat{f}\right)\left(P_{E}(X)\right) \quad X \in B^{2} .
$$


If the same function $f$ has two expressions $f=\widehat{f}_{1} \circ P_{E_{1}}=\widehat{f}_{2} \circ P_{E_{2}}$, with $E_{1}$ and $E_{2}$ in $\mathcal{F}\left(B^{\prime}\right), \widehat{f}_{1}$ in $\mathcal{S}_{E_{1}}$ and $\widehat{f}_{2}$ in $\mathcal{S}_{E_{2}}$, one checks that the right term of (65) is the same, be it computed with $E_{1}$ or $E_{2}$, which justifies the notation $T_{h} f$. According to the preceding, one has

$$
\left\|T_{h} f\right\|_{L^{2}\left(B^{2}, \mu_{B^{2}, h}\right)}=\left\|\widehat{T}_{h} \widehat{f}\right\|_{L^{2}\left(E^{2}, \mu_{E^{2}, h}\right)}=\|f\|_{L^{2}\left(B, \mu_{B, h / 2}\right)}=\|\widehat{f}\|_{L^{2}\left(E, \mu_{E, h / 2}\right)} .
$$

By density, $T_{h}$ extends as a partial isometry from $L^{2}\left(B, \mu_{B, h / 2}\right)$ in $L^{2}\left(B^{2}, \mu_{B^{2}, h}\right)$, denoted by $T_{h}$ as well. One sees that, for $f \in \mathcal{D}_{E}, T_{h} f$ is anti-holomorphic if one identifies $(x, \xi)$ and $x+i \xi$. This application $T_{h}$ is not surjective. One can find in Driver Hall $[\mathrm{D}-\mathrm{H}]$ and Hall $[\mathrm{HA}]$ a study of its image.

We shall need norms for the elements of $\mathcal{D}$. For all $E$ in $\mathcal{F}\left(B^{\prime}\right)$, for all $f$ in $\mathcal{D}_{E}$, written as $f=\widehat{f} \circ P_{E}$, with $\widehat{f}$ in $\mathcal{S}_{E}$, for every integer $m \geq 0$ and every positive $h$, one sets:

$$
I_{E, m, h}(f)=(2 \pi h)^{-\operatorname{dim}(E)} \int_{E^{2}}\left|\left(\widehat{T}_{h} \widehat{f}\right)(X)\right|(1+|X|)^{m} e^{-\frac{1}{4 h}|X|^{2}} d \lambda(X) .
$$

This integral is convergent, for, according to (64):

$$
I_{E, m, h}(f)=(2 \pi h)^{-\operatorname{dim}(E)} \int_{E^{2}}\left|<\gamma_{E, h / 2} \widehat{f}, \Psi_{X, h}^{E, L e b}>_{L e b}\right|(1+|X|)^{m} d \lambda(X) .
$$

Since the function $\gamma_{E, h / 2} \widehat{f}$ is in the Schwartz space $\mathcal{S}(E)$, it is well known that this integral is convergent.

For all $\widehat{f}$ in $\mathcal{S}_{E}$ one can write, in the sense of integrals of functions valued in $L^{2}\left(B, \mu_{B, h / 2}\right)$,

$$
\widehat{f}=(2 \pi h)^{-\operatorname{dim}(E)} \int_{E^{2}} e^{-\frac{1}{4 h}|X|^{2}}\left(\widehat{T}_{h} \widehat{f}\right)(X) \Psi_{X, h} d \lambda(X) .
$$

The equality (68) follows from (61), (64) and from the classical property, which makes sense since $\gamma_{E, h / 2} \widehat{f}$ is in the Schwartz space $\mathcal{S}(E)$ :

$$
\gamma_{E, h / 2} \widehat{f}=(2 \pi h)^{-\operatorname{dim}(E)} \int_{E^{2}}<\gamma_{E, h / 2} \widehat{f}, \Psi_{X, h}^{E, L e b}>_{L e b} \Psi_{X, h}^{E, L e b} d \lambda(X) .
$$

We will need the following function, for all $X=(x, \xi), Y=(y, \eta)$ and $Z=(z, \zeta)$ in $H^{2}$ :

$$
K_{h}^{A W}(X, Y, Z)=e^{\frac{1}{2 h}((x+i \xi) \cdot(z-i \zeta)+(y-i \eta) \cdot(z+i \zeta))} .
$$

Proposition 4.6. Let $E$ be in $\mathcal{F}\left(B^{\prime}\right)$. Suppose that $E$ decomposes as $E=E_{1} \oplus E_{2}$, where $E_{1}$ and $E_{2}$ in $\mathcal{F}\left(B^{\prime}\right)$ are mutually orthogonal. One considers two functions $\widehat{f}$ and $\widehat{g}$ in $\mathcal{S}_{E}$. One identifies $\widehat{T}_{h} \widehat{f}$ with a function $\left(\widehat{T}_{h} \widehat{f}\right)\left(X_{1}, X_{2}\right)$ on $\left(E_{1}\right)^{2} \times\left(E_{2}\right)^{2}$ and proceeds similarly for $\widehat{T}_{h} \widehat{g}$. Then, for all $Z_{1}$ in $E_{1}$ and all $X_{2}$ and $Y_{2}$ in $E_{2}$ :

$$
\int_{\left(E_{1}\right)^{4}} K_{h}^{A W}\left(X_{1}, Y_{1}, Z_{1}\right)\left(\widehat{T}_{h} \widehat{f}\right)\left(X_{1}, X_{2}\right) \overline{\left(\widehat{T}_{h} \widehat{g}\right)\left(Y_{1}, Y_{2}\right)} d \mu_{\left(E_{1}\right)^{4}, h}\left(X_{1}, Y_{1}\right)=\left(\widehat{T}_{h} \widehat{f}\right)\left(Z_{1}, X_{2}\right) \overline{\left(\widehat{T}_{h} \widehat{g}\right)\left(Z_{1}, Y_{2}\right)}
$$

Proof. For all $X_{2}=\left(x_{2}, \xi_{2}\right)$ in $\left(E_{2}\right)^{2}$ and all $t$ in $E_{1}$ set:

$$
\varphi_{X_{2}}(t)=e^{-\frac{1}{4 h}\left(x_{2}-i \xi_{2}\right)^{2}} \int_{E_{2}} \widehat{f}(t, y) e^{\frac{1}{h} y \cdot\left(x_{2}-i \xi_{2}\right)} d \mu_{E_{2}, h / 2}(y) .
$$

One can apply (63), replacing $\varphi$ with $\varphi_{X_{2}}$ and $E$ with $E_{1}$. One gets:

$$
\left(\widehat{T}_{h} \widehat{f}\right)\left(Z_{1}, X_{2}\right)=\int_{\left(E_{1}\right)^{2}} e^{\frac{1}{2 h}\left(x_{1}+i \xi_{1}\right) \cdot\left(z_{1}-i \zeta_{1}\right)}\left(\widehat{T}_{h} \widehat{f}\right)\left(X_{1}, X_{2}\right) d \mu_{\left(E_{1}\right)^{2}, h}\left(X_{1}\right) .
$$

By a similar treatment of $g$, one deduces (70) 


\subsection{Wigner Gaussian function}

The function $\widehat{H}_{h}^{\text {Gauss }}(\varphi, \psi)$ has been defined in (9) for functions $\varphi$ and $\psi$ in $\mathcal{S}_{E},(E \in \mathcal{F}(H))$. We well see below that this definition extends to $\varphi$ and $\psi$ in $L^{2}\left(E, \mu_{E, h / 2}\right)$. This is the case for the function $\Psi_{X, h}(X=(x, \xi))$ defined in (58), which is in $L^{2}\left(E, \mu_{E, h / 2}\right)$ if $E$ contains $x$ and $\xi$. We shall compute $\widehat{H}_{h}^{\text {Gauss }}(\varphi, \psi)$ (for $\varphi$ and $\psi$ in $\mathcal{S}_{E}$ ) using the Segal-Bargmann transforms of $\varphi$ and $\psi$.

\section{Proposition 4.7.}

1. For every subspace $E$ in $\mathcal{F}(H)$, for all $\varphi$ and $\psi$ in $L^{2}\left(E, \mu_{E, h / 2}\right)$, the equality (9) defines a function $\widehat{H}_{h}^{\text {Gauss }}(\varphi, \psi)$ continuous on $E^{2}$ and satisfying, for all $Z$ in $E^{2}$ :

$$
\left|\widehat{H}_{h}^{\text {Gauss }}(\varphi, \psi)(Z)\right| \leq e^{\frac{1}{h}|Z|^{2}}\|\varphi\|_{L^{2}\left(E, \mu_{E, h / 2}\right)}\|\psi\|_{L^{2}\left(E, \mu_{E, h / 2}\right)} .
$$

2. For all $X, Y$ and $Z$ in $H$ one can write:

$$
\widehat{H}_{h}^{\text {Gauss }}\left(\Psi_{X, h}, \Psi_{Y, h}\right)(Z)=e^{-\frac{1}{4 h}\left(|X|^{2}+|Y|^{2}\right)} K_{h}^{W e y l}(X, Y, Z)
$$

where one sets, for all $X=(x, \xi), Y=(y, \eta)$ and $Z=(z, \zeta)$ in $H^{2}$ :

$$
K_{h}^{W e y l}(X, Y, Z)=e^{\frac{1}{h}\left((x+i \xi) \cdot(z-i \zeta)+(y-i \eta) \cdot(z+i \zeta)-\frac{1}{2}(x+i \xi) \cdot(y-i \eta)\right)}
$$

3. Let $\varphi$ and $\psi$ be in $\mathcal{S}_{E}(E \in \mathcal{F}(H))$. Then the function $\widehat{H}_{h}^{\text {Gauss }}(\varphi, \psi)$ satisfies, for all $Z$ in $E^{2}$ :

$$
\widehat{H}_{h}^{\text {Gauss }}(\varphi, \psi)(Z)=\int_{E^{4}} K_{h}^{W e y l}(X, Y, Z)\left(\widehat{T}_{h} \varphi\right)(X) \overline{\left(\widehat{T}_{h} \psi\right)(Y)} d \mu_{E^{4}, h}(X, Y)
$$

Proof. Point 1) We begin by proving (71) for $\varphi$ and $\psi$ in $\mathcal{S}_{E}$. For all $Z=(z, \zeta)$ in $E^{2}$, by Cauchy Schwarz,

$$
\begin{aligned}
\left|\widehat{H}_{h}^{\text {Gauss }}(\varphi, \psi)(z, \zeta)\right| & \leq e^{\frac{1}{h}|\zeta|^{2}} \int_{E}\left|\varphi(z+t) e^{-z \cdot t / h}\right|\left|\psi(z-t) e^{z \cdot t / h}\right| d \mu_{E, h / 2}(t) \\
& \leq e^{\frac{1}{h}|\zeta|^{2}}\left(\int_{E}|\varphi(z+t)|^{2} e^{-2 z \cdot t / h} d \mu_{E, h / 2}(t) \int_{E}|\psi(z+t)|^{2} e^{-2 z \cdot t / h} d \mu_{E, h / 2}(t)\right)^{1 / 2} .
\end{aligned}
$$

Now,

$$
\int_{E}|\varphi(z+t)|^{2} e^{-2 z \cdot t / h} d \mu_{E, h / 2}(t)=e^{|z|^{2} / h} \int_{E}|\varphi(t)|^{2} d \mu_{E, h / 2}(t) .
$$

Point 2) For all $p$ and $q$ in the complexified $H_{\mathbf{C}}$ of $H$, we remark that the Wigner Gauss transform of

$$
f(t)=e^{p \cdot t} \quad g(t)=e^{q \cdot t} \quad t \in H
$$

satisfies, for all $Z=(z, \zeta)$ in $H^{2}$

$$
\widehat{H}_{h}^{\text {Gauss }}(f, g)(z, \zeta)=e^{\frac{h}{4}(p-\bar{q})^{2}+z \cdot(p+\bar{q})-i \zeta \cdot(p-\bar{q})} .
$$

To establish point 2), setting $X=(x, \xi), Y=(y, \eta)$, it suffices to apply (75) to $p=(x+i \xi) / h, q=(y+i \eta) / h$.

Point 3) Remark that, according to (71), for all $Z$ in $E^{2}$, the application $(\varphi, \psi) \rightarrow \widehat{H}_{h}^{\text {Gauss }}(\varphi, \psi)(Z)$ is a continuous bilinear form on $L^{2}\left(E, \mu_{E, h / 2}\right) \times L^{2}\left(E, \mu_{E, h / 2}\right)$. Moreover, for all $\varphi$ in $\mathcal{S}_{E}$, the application associating $e^{-\frac{1}{4 h}|X|^{2}}\left(\widehat{T}_{h} \varphi\right)(X) \widetilde{\Psi}_{X, h}$ with $X \in E^{2}$ is integrable, in the Bochner sense, with values in $L^{2}\left(E, \mu_{B, h / 2}\right)$, and its integral is given by (68). The same holds for $\psi$. It yields, as in Corollary 2 in Section V.5 in Yosida [Y]:

$$
\widehat{H}_{h}^{\text {Gauss }}(\varphi, \psi)(Z)=(2 \pi h)^{-2 \operatorname{dim}(E)} \int_{E^{4}} e^{-\frac{1}{4 h}\left(|X|^{2}+|Y|^{2}\right)} \widehat{H}_{h}^{\text {Gauss }}\left(\Psi_{X, h}, \Psi_{Y, h}\right)(Z)\left(\widehat{T}_{h} \varphi\right)(X) \overline{\left(\widehat{T}_{h} \psi\right)(Y)} d \lambda(X, Y) .
$$


The equality (74) follows from that, according to (72).

The Wigner-Gauss transform $H_{h}^{\text {Gauss }}(f, g)$ of two functions $f$ and $g$ in $\mathcal{D}_{E}\left(E\right.$ in $\left.\mathcal{F}\left(B^{\prime}\right)\right)$ is defined by (11) if $f=\widehat{f} \circ P_{E}$ and $g=\widehat{g} \circ P_{E}$, with $\widehat{f}$ and $\widehat{g}$ in $\mathcal{S}_{E}$. One has:

$$
H_{h}^{\text {Gauss }}(f, g)(Z)=\int_{E^{4}} \widetilde{K}_{h}^{W e y l}(X, Y, Z)\left(\widehat{T}_{h} \widehat{f}\right)(X) \overline{\left(\widehat{T}_{h} \widehat{g}\right)(Y)} d \mu_{E^{4}, h}(X, Y) \quad \text { a.e.Z } \in B^{2}
$$

with:

$$
\widetilde{K}_{h}^{W e y l}(X, Y, Z)=e^{\frac{1}{h}\left(\ell_{x+i \xi}(z-i \zeta)+\ell_{y-i \eta}(z+i \zeta)-\frac{1}{2}(x+i \xi) \cdot(y-i \eta)\right)} \quad(X, Y) \in H^{2} \text {, a.e. } Z \in B^{2} .
$$

Indeed, according to (73), (77) and (51), one has, for all $X$ and $Y$ in $E^{2}$, for almost every $Z$ in $B^{2}$

$$
K_{h}^{W e y l}\left(X, Y, P_{E}(Z)\right)=\widetilde{K}_{h}^{W e y l}(X, Y, Z) .
$$

Note that

$$
H_{h}^{\text {Gauss }}(f, 1)(Z)=\left(T_{h} f\right)(2 Z) .
$$

Let us now investigate the bounds on the norm and the density extensions of the Wigner-Gauss transformation.

Proposition 4.8. For all $f$ and $g$ in $\mathcal{D}$, the Gaussian Wigner function $H_{h}^{\text {Gauss }}(f, g)$ is in $L^{1}\left(B^{2}, \mu_{B^{2}}, h / 2\right)$. The operator which associates, with all functions $f$ and $g$ in $\mathcal{D}, H_{h}^{\text {Gauss }}(f, g)$, extends uniquely as a continuous bilinear application with norm $\leq 1$, from $L^{2}\left(B, \mu_{B, h / 2}\right) \times L^{2}\left(B, \mu_{B, h / 2}\right)$ to $L^{2}\left(B^{2}, \mu_{B^{2}, h / 4}\right)$. The operator which associates, with all functions $f$ and $g$ in $\mathcal{D}, H_{h}^{\text {Gauss }}(f, g)$, extends uniquely as a continuous bilinear application from $L^{2}\left(B, \mu_{B, h / 2}\right) \times L^{2}\left(B, \mu_{B, h / 2}\right)$ to the space of continuous functions on $H^{2}$ and for all $Z$ in $H^{2}$ :

$$
\left|H_{h}^{\text {Gauss }}(f, g)(Z)\right| \leq e^{\frac{1}{h}|Z|^{2}}\|f\|_{L^{2}\left(B, \mu_{B, h / 2}\right)}\|g\|_{L^{2}\left(B, \mu_{B, h / 2}\right)} .
$$

Proof. Let $f$ and $g$ be in $\mathcal{D}$, let $E$ be such that $f$ and $g$ are in $\mathcal{D}_{E}$ and let $\widehat{f}$ and $\widehat{g}$ be functions defined on $E$ such that $f=\widehat{f} \circ P_{E}$ and $g=\widehat{g} \circ P_{E}$. One knows that $H_{h}^{\text {Gauss }}(f, g)=\widehat{H}_{h}^{\text {Gauss }}(\widehat{f}, \widehat{g}) \circ P_{E}$, where $\widehat{H}_{h}^{\text {Gauss }}(\widehat{f}, \widehat{g})$ satisfies (10). If the functions $f$ and $g$ are in $\mathcal{D}_{E}$, the functions $\gamma_{E, h / 2} \widehat{f}$ and $\gamma_{E, h / 2} \widehat{g}$ are in $\mathcal{S}(E)$. According to Folland [F] (Proposition 1.92), the function $H_{h}^{L e b}\left(\gamma_{E, h / 2} \widehat{f}, \gamma_{E, h / 2} \widehat{g}\right)$ is in $\mathcal{S}\left(E^{2}\right)$, hence in $L^{1}\left(E^{2}, \lambda\right)$. We deduce from that, according to (10), that $\widehat{H}_{h}^{\text {Gauss }}(\widehat{f}, \widehat{g})$ is in $L^{1}\left(E^{2}, \mu_{E^{2}, h / 2}\right)$. The first point of the proposition follows as a consequence, according to (77). The functions $\gamma_{E, h / 2} \widehat{f}$ and $\gamma_{E, h / 2} \widehat{g}$ are in $L^{2}(E, \lambda)$, where $\lambda$ is the Lebesgue measure on E. According to Folland [F] (Proposition 1.92), one has

$$
\begin{aligned}
\left\|H_{h}^{L e b}\left(\gamma_{E, h / 2} \widehat{f}, \gamma_{E, h / 2} \widehat{g}\right)\right\|_{L^{2}\left(E^{2}, \lambda\right)} & \leq(2 \pi h)^{\operatorname{dim}(E) / 2}\left\|\gamma_{E, h / 2} \widehat{f}\right\|_{L^{2}(E, \lambda)}\left\|\gamma_{E, h / 2} \widehat{f}\right\|_{L^{2}(E, \lambda)} \\
& =(2 \pi h)^{\operatorname{dim}(E) / 2}\|\widehat{f}\|_{L^{2}\left(E, \mu_{E, h / 2}\right)}\|\widehat{g}\|_{L^{2}\left(E, \mu_{E, h / 2}\right)}
\end{aligned}
$$

It follows that, according to (10):

$$
\left\|\widehat{H}_{h}^{\text {Gauss }}(\widehat{f}, \widehat{g})\right\|_{L^{2}\left(E^{2}, \mu_{E^{2}, h / 4}\right)} \leq\|\widehat{f}\|_{L^{2}\left(E, \mu_{E, h / 2}\right)}\|\widehat{g}\|_{L^{2}\left(E, \mu_{E, h / 2}\right)} .
$$

Hence, according to (7):

$$
\left\|H_{h}^{\text {Gauss }}(f, g)\right\|_{L^{2}\left(B^{2}, \mu_{B^{2}, h / 4}\right)} \leq\|f\|_{L^{2}\left(B, \mu_{B, h / 2}\right)}\|g\|_{L^{2}\left(B, \mu_{B, h / 2}\right)} .
$$

The extension from $L^{2}\left(B, \mu_{B, h / 2}\right) \times L^{2}\left(B, \mu_{B, h / 2}\right)$ in the space of continuous functions on $H^{2}$ is a consequence of (71).

The extensions of the Wigner-Gauss transformation will also be denoted by $H_{h}^{\text {Gauss }}$. For all $X$ and $Y$ in $H^{2}$, for almost $\mu_{B^{2}, h / 4}$ all $Z$ in $B^{2}$ one has:

$$
H_{h}^{\text {Gauss }}\left(\widetilde{\Psi}_{X, h}, \widetilde{\Psi}_{Y, h}\right)(Z)=e^{-\frac{1}{4 h}\left(|X|^{2}+|Y|^{2}\right)} \widetilde{K}_{h}^{W e y l}(X, Y, Z)
$$

One verifies that this function (of $Z$ ) has a $L^{2}\left(B^{2}, \mu_{B^{2}, h / 4}\right)$ norm equal to 1 .

The following proposition will be useful in Section 7 . 
Proposition 4.9. If $\left(E_{n}\right)$ is an increasing sequence of subspaces in $\mathcal{F}\left(B^{\prime}\right)$, whose union is dense in $H$, then for all $X$ and $Y$ in $H^{2}$ :

$$
\lim _{n \rightarrow+\infty} H_{h}^{\text {Gauss }}\left(\Psi_{X, h} \circ P_{E_{n}}, \Psi_{Y, h} \circ P_{E_{n}}\right)(Z)=e^{-\frac{1}{4 h}\left(|X|^{2}+|Y|^{2}\right)} \widetilde{K}_{h}^{W e y l}(X, Y, Z) .
$$

The convergence (as a function of $Z$ ) is in the sense of $L^{2}\left(B^{2}, \mu_{B^{2}, h / 2}\right)$.

Proof. According to (11) and (72),

$$
H_{h}^{\text {Gauss }}\left(\Psi_{X, h} \circ P_{E_{n}}, \Psi_{Y, h} \circ P_{E_{n}}\right)(Z)=e^{-\frac{1}{4 h}\left(|X|^{2}+|Y|^{2}\right)} K_{h}^{W e y l}\left(X, Y, P_{E_{n}}(Z)\right) .
$$

Thanks to the definition (73) and to (49), one proves that

$$
\lim _{n \rightarrow \infty} K_{h}^{W e y l}\left(X, Y, P_{E_{n}}(Z)\right)=\widetilde{K}_{h}^{W e y l}(X, Y, Z),
$$

the limit being taken in $L^{2}\left(B^{2}, \mu_{B^{2}, h / 2}\right)$. This proves the proposition.

\subsection{Convergence in Definition 1.2 .}

If the function $\widetilde{F}$ is bounded and if $f$ and $g$ are in $\mathcal{D}$, the expression $Q_{h}^{W e y l}(\widetilde{F})(f, g)$ from Definition 1.2 is well defined, according to Proposition 4.8. We show that the same property is true under the weaker condition that, for a given $m \geq 0$, the norm $N_{m}(\widetilde{F})$ in (12) is finite. This condition is satisfied, for example, for functions of the form $\widetilde{F}(x, \bar{\xi})=\prod \ell_{a_{j}}(x)^{\alpha_{j}} \prod \ell_{b_{k}}(\xi)^{\beta_{k}}$, with $a_{j}$ and $b_{j}$ in $H$, using (45). One can show that $N_{m}(\widetilde{F})<\infty$, with $m=\sum \alpha_{j}+\sum \beta_{k}$.

Proposition 4.10. Let $\widetilde{F}$ be a Borel function on $B^{2}$, such that the norm $N_{m}(\widetilde{F})$ defined in (12) is finite for a nonnegative integer $m$. For each subspace $E \in \mathcal{F}\left(B^{\prime}\right)$, for all $f$ and $g$ in $\mathcal{D}_{E}$, we have:

$$
\left|Q_{h}^{W e y l}(\widetilde{F})(f, g)\right| \leq I_{E, m, h}(f) I_{E, m, h}(g) N_{m}(\widetilde{F}),
$$

where $I_{E, m, h}(f)$ is defined in 67 .

Proof. Let $f$ and $g$ be in $\mathcal{D}_{E}$. According to Definition 1.2, to (11) and to Proposition 4.7, we only have to check that the function:

$$
(X, Y, Z) \rightarrow\left|\widetilde{K}_{h}^{W e y l}(X, Y, Z)\right|\left|\left(\widehat{T_{h}} \widehat{f}\right)(X)\right|\left|\left(\widehat{T_{h}} \widehat{g}\right)(Y)\right||\widetilde{F}(Z)|
$$

is in $L^{1}\left(E^{4} \times B^{2}\right)$ for the measure $d \mu_{E^{4}, h}(X, Y) d \mu_{B^{2}, h / 2}(Z)$. To this aim, one applies the change of variables (46)) with $B$ replaced with $B^{2}, h$ with $h / 2, g(Z)=\left|\widetilde{F}(Z) \widetilde{K}_{h}^{W e y l}(X, Y, Z)\right|$ and $a=(X+Y) / 2$. Note that

$$
\widetilde{K}_{h}^{W e y l}\left(X, Y, Z+\frac{X+Y}{2}\right) e^{-\frac{1}{h} \ell_{X+Y}(Z)-\frac{1}{4 h}|X+Y|^{2}}=e^{\frac{1}{4 h}\left(|X|^{2}+|Y|^{2}\right)} e^{\frac{i}{h} \varphi(X, Y, Z)}
$$

with

$$
\varphi(X, Y, Z)=\frac{1}{2} \sigma(X, Y)+\ell_{\xi-\eta}(z)-\ell_{x-y}(\zeta)
$$

where $\sigma$ is the symplectic form. Consequently, for all $X$ and $Y$ in $H$ :

$$
\int_{B^{2}}\left|\widetilde{K}_{h}^{W e y l}(X, Y, Z)\right||\widetilde{F}(Z)| d \mu_{B^{2}, h / 2}(Z) \leq e^{\frac{1}{4 h}\left(|X|^{2}+|Y|^{2}\right)} \int_{B^{2}}\left|\widetilde{F}\left(Z+\frac{X+Y}{2}\right)\right| d \mu_{B^{2}, h / 2}(Z) .
$$

Hence, for every integer $m$ such that the norm $N_{m}(\widetilde{F})$ is finite,

$$
\int_{B^{2}}\left|\widetilde{K}_{h}^{W e y l}(X, Y, Z)\right||\widetilde{F}(Z)| d \mu_{B^{2}, h / 2}(Z) \leq e^{\frac{1}{4 h}\left(|X|^{2}+|Y|^{2}\right)}\left(1+\frac{|X+Y|}{2}\right)^{m} N_{m}(\widetilde{F}) .
$$


The function (83) is indeed in $L^{1}\left(E^{4} \times B^{2}\right)$ for the announced measure. Therefore

$$
\left|Q_{h}^{W e y l}(\widetilde{F})(f, g)\right| \leq N_{m}(\widetilde{F}) \int_{E^{4}} e^{\frac{1}{4 h}\left(|X|^{2}+|Y|^{2}\right)}\left(1+\frac{|X+Y|}{2}\right)^{m}\left|\left(\widehat{T_{h} f}\right)(X)\right|\left|\left(\widehat{T_{h} g}\right)(Y)\right| d \mu_{E^{4}, h}(X, Y) .
$$

This proves the claim.

This implies that the quadratic form in (13) is well defined if $\widetilde{F}$ is bounded or if there exists an integer $m \geq 0$ such that $N_{m}(\widetilde{F})$ is finite.

One can write, for all $f$ and $g$ in $\mathcal{D}_{E}$ :

$$
Q_{h}^{W e y l}(\widetilde{F})(f, g)=\int_{E^{4} \times B^{2}} \widetilde{K}_{h}^{W e y l}(X, Y, Z)\left(\widehat{T_{h}} \widehat{f}\right)(X) \overline{\left(\widehat{T_{h}} \widehat{g}\right)(Y)} \widetilde{F}(Z) d \mu_{E^{4}, h}(X, Y) d \mu_{B^{2}, h / 2}(Z) .
$$

\subsection{Anti-Wick and hybrid operators}

We now rewrite the hybrid operator of Definition 2.2 in a form more suitable for norm estimates. At the same time we shall rewrite the anti-Wick operator defined by (23) and give an expression nearer to the usual definition. One will use the measure $\nu_{B^{2}, E^{2}, h_{1}, h_{2}}$ defined in (53).

Proposition 4.11. Let $f$ and $g$ be in $\mathcal{D}$. Let $D$ be in $\mathcal{F}\left(B^{\prime}\right), \widehat{f}$ and $\widehat{g}$ in $\mathcal{S}_{D}$ be such that $f=\widehat{f} \circ P_{D}$ and $g=\widehat{g} \circ P_{D}$. For every Borel function $\widetilde{F}$ bounded on $B^{2}$, for every subspace $E$ in $\mathcal{F}\left(B^{\prime}\right)$ :

$$
Q_{h}^{h y b, E}(\widetilde{F})(f, g)=\int_{D^{2} \times D^{2} \times B^{2}} K_{h}^{h y b, E}(X, Y, Z)\left(\widehat{T}_{h} \widehat{f}\right)(X) \overline{\left(\widehat{T}_{h} \widehat{g}\right)(Y)} \widetilde{F}(Z) d \mu_{D^{4}, h}(X, Y) d \nu_{B^{2}, E^{2}, h / 2, h}(Z)
$$

where $K_{h}^{h y b, E}$ is the function defined for all $(X, Y)$ in $H^{4}$ and almost every $Z$ in $B^{2}$, by:

$$
K_{h}^{h y b, E}(X, Y, Z)=\widetilde{K}_{h}^{W e y l}\left(X_{E}, Y_{E}, Z_{E}\right) \widetilde{K}_{h}^{A W}\left(X_{E^{\perp}}, Y_{E^{\perp}}, Z_{E^{\perp}}\right) .
$$

Here we set $X=\left(X_{E}, X_{E^{\perp}}\right), \ldots$ according to Proposition 2.1, $\widetilde{K}_{h}^{W e y l}$ has been defined by (77) and

$$
\widetilde{K}_{h}^{A W}(X, Y, Z)=e^{\frac{1}{2 h}\left(\ell_{x+i \xi}\left((z-i \zeta)+\ell_{y-i \eta}(z+i \zeta)\right)\right.} .
$$

The above integral does not depend on the subspace $D$ such that $f$ and $g$ are in $\mathcal{D}_{D}($ for $f$ and $g$ in $\mathcal{D}$.)

Proof. According to Definitions 2.2 and 1.2 , one has

$$
Q_{h}^{h y b, E}(\widetilde{F})(f, g)=\int_{B^{2}}\left(\widetilde{H}_{E^{\perp}, h / 2} \widetilde{F}\right)(Z) G(Z) d \mu_{B^{2}, h / 2}(Z)
$$

with

$$
G(Z)=H_{h}^{\text {Gauss }}(f, g)(Z) .
$$

This function $G$ belongs to $L^{1}\left(B^{2}, \mu_{B^{2}, h / 2}\right)$ thanks to Proposition 4.8 According to Proposition 4.5 applied with $p=+\infty, q=1, h_{1}=h_{2}=h / 2$ and $t=h / 2$, we obtain:

$$
Q_{h}^{h y b, E}(\widetilde{F})(f, g)=\int_{B^{2}} \widetilde{F}(Z)\left(\widetilde{M}_{E^{\perp}, h / 2, h / 2, h / 2} G\right)(Z) d \nu_{B^{2}, E^{2}, h / 2, h}(Z) .
$$

According to (76), the form of $f$ and $g$ imply that the function $G=H_{h}^{\text {Gauss }}(f, g)$ has the following expression:

$$
G(Z)=\int_{D^{4}} \widetilde{K}_{h}^{W e y l}(X, Y, Z)\left(\widehat{T}_{h} \widehat{f}\right)(X) \overline{\left(\widehat{T}_{h} \widehat{g}\right)(Y)} d \mu_{D^{4}, h}(X, Y) .
$$


Using the expression (54) of $\widetilde{M}_{E^{\perp}, h / 2, h / 2, h / 2}$ and Fubini's theorem, we obtain:

$$
\left(\widetilde{M}_{E^{\perp}, h / 2, h / 2, h / 2} G\right)(Z)=\int_{D^{4}} \Phi(X, Y, Z)\left(\widehat{T_{h}} \widehat{f}\right)(X) \overline{\left(\widehat{T_{h}} \widehat{g}\right)(Y)} d \mu_{D^{4}, h}(X, Y)
$$

where

$$
\begin{aligned}
\Phi(X, Y, Z) & =\int_{\left(E^{\perp}\right)^{2}} \widetilde{K}_{h}^{W e y l}\left(X, Y,\left(Z_{E}, V+\frac{1}{2} Z_{E^{\perp}}\right)\right) d \mu_{\left(E^{\perp}\right)^{2}, \frac{h}{4}}(V) \\
& =\widetilde{K}_{h}^{W e y l}\left(X_{E}, Y_{E}, Z_{E}\right) \int_{\left(E^{\perp}\right)^{2}} \widetilde{K}_{h}^{W e y l}\left(X_{E^{\perp}}, Y_{E^{\perp}}, V+\frac{1}{2} Z_{E^{\perp}}\right) d \mu_{\left(E^{\perp}\right)^{2}, \frac{h}{4}}(V) .
\end{aligned}
$$

Here the functions $\ell_{x}, \ldots, \ell_{\eta}$ appearing in the kernels are linear, since $x, \ldots, \eta$ belong to $D \subset B^{\prime}$. One checks, using (43), (with $B$ replaced with $\left(E^{\perp}\right)^{2}$ and $h$ with $h / 4$ ), that:

$$
\int_{\left(E^{\perp}\right)^{2}} \widetilde{K}_{h}^{W e y l}\left(X_{E^{\perp}}, Y_{E^{\perp}}, V+\frac{1}{2} Z_{E^{\perp}}\right) d \mu_{\left(E^{\perp}\right)^{2}, \frac{h}{4}}(V)=K_{h}^{A W}\left(X_{E^{\perp}}, Y_{E^{\perp}}, Z_{E^{\perp}}\right) .
$$

The proposition follows from that.

Corollary 4.12. Let $f$ and $g$ be in $\mathcal{D}$. Let $D$ be in $\mathcal{F}\left(B^{\prime}\right), \widehat{f}$ and $\widehat{g}$ in $\mathcal{S}_{D}$ such that $f=\widehat{f} \circ P_{D}$ and $g=\widehat{g} \circ P_{D}$. For every Borel function $\widetilde{F}$, bounded on $B^{2}$, one has:

$$
Q_{h}^{A W}(\widetilde{F})(f, g)=\int_{B^{2}} \widetilde{F}(Z)\left(\widehat{T}_{h} \widehat{f}\right)\left(P_{D}(Z)\right) \overline{\left(\widehat{T}_{h} \widehat{g}\right)\left(P_{D}(Z)\right)} d \mu_{B^{2}, h}(Z) .
$$

There exists an operator $O p_{h}^{A W}(\widetilde{F})$, bounded in $L^{2}\left(B, \mu_{B, h / 2}\right)$, such that

$$
Q_{h}^{A W}(\widetilde{F})(f, g)=<O p_{h}^{A W}(\widetilde{F}) f, g>.
$$

The norm of this operator is smaller than $\|\widetilde{F}\|_{\infty}$.

Proof. One applies Proposition 4.11 with $E=\{0\}, E^{\perp}=B$. This yields

$$
Q_{h}^{A W}(\widetilde{F})(f, g)=\int_{D^{4} \times B^{2}} \widetilde{K}_{h}^{A W}(X, Y, Z)\left(\widehat{T}_{h} \widehat{f}\right)(X) \overline{\left(\widehat{T}_{h} \widehat{g}\right)(Y)} \widetilde{F}(Z) d \mu_{D^{4}, h}(X, Y) d \mu_{B^{2}, h}(Z) .
$$

According to Proposition 4.6, with $E_{1}$ replaced with $D$ and $E_{2}$ with $\{0\}$, one has:

$$
\int_{D^{4}} \widetilde{K}_{h}^{A W}(X, Y, Z)\left(\widehat{T}_{h} \widehat{f}\right)(X) \overline{\left(\widehat{T}_{h} \widehat{g}\right)(Y)} d \mu_{D^{4}, h}(X, Y)=\left(\widehat{T}_{h} \widehat{f}\right)\left(P_{D}(Z)\right) \overline{\left(\widehat{T}_{h} \widehat{g}\right)\left(P_{D}(Z)\right)},
$$

since $\widetilde{K}_{h}^{A W}(X, Y, Z)=K_{h}^{A W}\left(X, Y, P_{D}(Z)\right)$. The corollary then follows, according to [66).

\subsection{Partial Heat semigroups and stochastic extensions.}

We shall first be concerned with establishing a property for the stochastic extension given by Definition 1.2 . Namely, we shall prove the fact that it commutes with heat operators acting on subspaces of finite dimension, provided that these operators act on bounded Lipschitz functions on $H$.

For all $E$ in $\mathcal{F}\left(B^{\prime}\right)$, and for all $t>0$, we shall use the operators $H_{E, t}$ and $\widetilde{H}_{E, t}$ both defined by (26), but the first one acting in spaces of functions on $H^{2}$, whereas the second one acts in spaces of functions on $B^{2}$.

Proposition 4.13. Let $F$ be a bounded Lipschitz function on $H^{2}$. Fix $h>0$ and $t>0$. Assume that $F$ has a stochastic extension $\widetilde{F}$ for the measure $\mu_{B, h}$. Then, for each $E$ in $\mathcal{F}\left(B^{\prime}\right)$, the function $H_{E, t} F$ has a stochastic extension for the measure $\mu_{B, h}$, and this stochastic extension is equal to $\widetilde{H}_{E, t} \widetilde{F}$. 
Proof. Let $\left(E_{n}\right)$ be an increasing sequence in $\mathcal{F}(H)$, whose union is dense in $H$. In order to obtain that $\left(H_{E, t} F\right) \circ P_{E_{n}}$ converges to $\widetilde{H}_{E, t} \widetilde{F}$ in $\mu_{B, h}-$ probability, it is necessary and sufficient to verify that, for all subsequences $\left(\left(H_{E, t} F\right) \circ P_{E_{\varphi}(n)}\right)_{n}$, one may extract a further subsequence which converges $\mu_{B, h \text {-almost }}$ everywhere to $\widetilde{H}_{E, t} \widetilde{F}$. Set $\psi: \mathbb{N} \rightarrow \mathbb{N}$ such that $\left(F \circ P_{E_{\varphi(\psi(n))}}\right)_{n}$ tends to $\widetilde{F} \mu_{B, h^{-a l m o s t} \text { everywhere. Set }}$ $G_{n}=E_{\varphi(\psi(n))}$. For $X \in B$,we have

$$
\begin{aligned}
& \left(H_{E, t} F\right) \circ P_{G_{n}}(X)-\widetilde{H}_{E, t} \widetilde{F}(X) \\
& =(2 \pi t)^{-\operatorname{dim}(E)} \int_{E^{2}} e^{-\frac{|Y|^{2}}{2 t}}\left(F\left(P_{G_{n}}(X)+Y\right)-F\left(P_{G_{n}}(X+Y)\right)\right) d \lambda(Y) \\
& +(2 \pi t)^{-\operatorname{dim}(E)} \int_{E^{2}} e^{-\frac{|Y|^{2}}{2 t}}\left(F\left(P_{G_{n}}(X+Y)\right)-\widetilde{F}(X+Y)\right) d \lambda(Y) .
\end{aligned}
$$

By assumption, there exists $K>0$ such that for all $(Z, V) \in H^{2}$ :

$$
|F(Z+V)-F(Z)| \leq K|V|,
$$

which shows, by the dominated convergence theorem, that the first term converges to 0 for all $X$.

We shall prove that the second term converges to 0 in $L^{1}\left(B, \mu_{B, h}\right)$, which will give the convergence $\mu_{B, h^{-}}$ almost everywhere for a subsequence. Set

$$
A_{n}=\int_{B^{2}}\left|\int_{E^{2}} e^{-\frac{|Y|^{2}}{2 t}}\left(F\left(P_{G_{n}}(X+Y)\right)-\widetilde{F}(X+Y)\right) d \lambda(Y)\right| d \mu_{B^{2}, h}(X) .
$$

Fubini's theorem and the decomposition (20), $\mu_{B, h}(X)=\mu_{E, h}\left(X_{E}\right) \otimes \mu_{\left(E^{\perp}\right), h}\left(X_{E^{\perp}}\right)$, give

$$
A_{n} \leq \int_{E^{4} \times\left(E^{\perp}\right)^{2}} e^{-\frac{|Y|^{2}}{2 t}}\left|F\left(P_{G_{n}}\left(X_{E}+Y, X_{E^{\perp}}\right)\right)-\widetilde{F}\left(X_{E}+Y, X_{E^{\perp}}\right)\right| d \lambda(Y) d \mu_{E^{2}, h}\left(X_{E}\right) d \mu_{\left(E^{\perp}\right)^{2}, h}\left(X_{E^{\perp}}\right) .
$$

Then the change of variables $X_{E}+Y \rightarrow Z$ and an explicit computation on gaussian functions yield:

$$
A_{n} \leq C(h, t) \int_{E^{2} \times\left(E^{\perp}\right)^{2}}\left|F\left(P_{G_{n}}\left(Z, X_{E^{\perp}}\right)\right)-\widetilde{F}\left(Z, X_{E^{\perp}}\right)\right| e^{|Z|^{2}\left(\frac{1}{2 h}-\frac{1}{2(t+h)}\right)} d \mu_{E^{2}, h}(Z) d \mu_{\left(E^{\perp}\right)^{2}, h}\left(X_{E^{\perp}}\right) .
$$

We return to $B$ setting $W=\left(Z, X_{E^{\perp}}\right)$. We have

$$
A_{n} \leq C(h, t) \int_{B^{2}}\left|F\left(P_{G_{n}}(W)\right)-\widetilde{F}(W)\right| e^{\left|P_{E}(W)\right|^{2}\left(\frac{1}{2 h}-\frac{1}{2(t+h)}\right)} d \mu_{B^{2}, h}(W) .
$$

The term in the above integral tends to $0 \mu_{B, h}$ almost everywhere and is bounded by

$$
\Psi(W)=2 e^{\left|P_{E}(W)\right|^{2}\left(\frac{1}{2 h}-\frac{1}{2(t+h)}\right)} \cdot \sup _{X \in H^{2}}|F(X)| .
$$

Using (7), an explicit computation shows that the bound $\Psi$ is in $L^{1}\left(B, \mu_{B, h}\right)$. The second term tends indeed to 0 in $L^{1}\left(B, \mu_{B, h}\right)$, thus almost everywhere for $\mu_{B, h}$, when extracting again a subsequence, indexed by $\zeta$. The

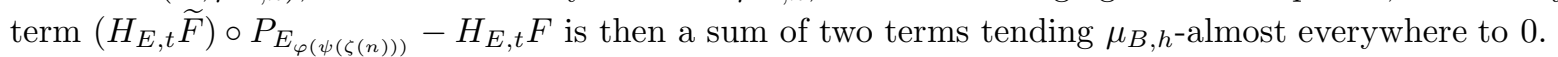

Using the Hilbertian basis $\left(e_{j}\right)_{(j \in \Gamma)}$ chosen for our construction, we defined in (32), for each finite subset $I$ in $\Gamma$, operators denoted by $\widetilde{T}_{I, h}$ and $\widetilde{S}_{I, h}$. These operators act in the space of bounded Borel functions on $B^{2}$. We denote by $T_{I, h}$ the operator defined similarly to (32), but acting in the space of bounded continuous functions on $H^{2}$.

Proposition 4.14. If $\widetilde{F}$ is a Borel function bounded on $B^{2}$ and is the stochastic extension for the measure $\mu_{B^{2}, h}$ of a function $F$ in $S_{1}(M, \varepsilon)$, the family $\left(\varepsilon_{j}\right)_{(j \in \Gamma)}$ being square summable, then $\widetilde{T}_{I, h} \widetilde{F}$ is the stochastic extension of the function $T_{I, h} F$ for the measure $\mu_{B^{2}, h}$. 
Proof. Since $T_{I, h}=\prod_{j \in I}\left(I-H_{e_{j}}\right)$, products like $\prod_{j \in J} H_{e_{j}}=H_{E, h / 2}$ appear, with $E=E(J)=\operatorname{Vect}\left(e_{j}, j \in\right.$ $J), J \subset I$. We then apply Proposition 4.13 for each of these terms, replacing $t$ with $h / 2$ and $E$ with $E(J)$, $J \subseteq I$. One may use this proposition since every function $F$ in $S_{1}(M, \varepsilon)$ verifies, for all $X$ and $V$ in $H^{2}$ :

$$
|F(X+V)-F(X)| \leq M|V| \sqrt{2}\left[\sum_{j \in \Gamma} \varepsilon_{j}^{2}\right]^{1 / 2} .
$$

Indeed, let $\eta>0$ and let $J$ be a finite subset of $\Gamma$ such that $\left|F(X+V)-F\left(X+P_{E(J)}(V)\right)\right|<\eta$. We obtain, using the hypothesis of differentiability along the $u_{j}$ and $v_{j}$,

$$
\left|F\left(X+P_{E(J)}(V)\right)-F(X)\right| \leq \sum_{j \in J} M \varepsilon_{j}\left(\left|V \cdot u_{j}\right|+\left|V \cdot v_{j}\right|\right) .
$$

Then

$$
|F(X+V)-F(X)| \leq \eta+M\left(2 \sum_{j \in \Gamma} \varepsilon_{j}^{2}\right)^{1 / 2}|V|,
$$

which gives the result.

\section{Proof of Proposition 3.1.}

Proposition 3.1 is a direct consequence of Propositions 5.2 and 5.3 below.

For every integer $m$ and for any subset $I$ in $\Gamma, \mathcal{M}_{m}(I)$ designates the set of mappings $(\alpha, \beta)$ from $I$ into $\{0,1, \ldots, m\} \times\{0,1, \ldots, m\}$. When $\left(e_{j}\right)_{(j \in \Gamma)}$ is the Hilbertian basis of $H$ chosen for our construction, we set $u_{j}=\left(e_{j}, 0\right)$ and $v_{j}=\left(0, v_{j}\right)$ for all $j \in \Gamma$. For each multi-index $(\alpha, \beta)$ in $\mathcal{M}_{m}(I)$, we set:

$$
\partial_{u}^{\alpha} \partial_{v}^{\beta}=\prod_{j \in I} \partial_{u_{j}}^{\alpha_{j}} \partial_{v_{j}}^{\beta_{j}}
$$

For any function $F$ in $S_{m}(M, \varepsilon),(m \geq 0)$, let us define :

$$
N_{I, h}^{(m)}(F)=\sum_{(\alpha, \beta) \in \mathcal{M}_{m}(I)} h^{(|\alpha|+|\beta|) / 2}\left\|\partial_{u}^{\alpha} \partial_{v}^{\beta} F\right\|_{\infty}
$$

where $\|\cdot\|_{\infty}$ denotes the supremum norm for bounded functions on $H^{2}$. For a function defined on a subspace $E$ of $H$, let $N_{I, h}^{(m)}(F)$ denote the same expression, the supremum being taken on $E$. Adapting the proof of Unterberger [U-2] or [A-J-N-1, we obtain the following proposition.

Proposition 5.1. Let $I$ be a finite subset of $\Gamma$ and $E=E(I)$ be the subspace spanned by the $e_{j}(j \in I)$. Let $H$ be a continuous function on $E^{2}$, such that $N_{I, h}^{(2)}(H)$ is well defined. We set, for all $U$ and $V$ in $L^{2}\left(E^{2}, \mu_{E^{2}, h}\right)$ :

$$
Q(U, V)=\int_{E^{6}} K_{h}^{W e y l}(X, Y, Z) U(X) V(Y) H(Z) d \mu_{E^{4}, h}(X, Y) d \mu_{E^{2}, h / 2}(Z)
$$

Then, we have:

$$
|Q(U, V)| \leq\left(\frac{9 \pi}{2}\right)^{|I|} N_{I, h}^{(2)}(H)\|U\|_{L^{2}\left(E^{2}, \mu_{E^{2}, h}\right)}\|V\|_{L^{2}\left(E^{2}, \mu_{E^{2}, h}\right)}
$$


Proof. We shall study the following integral kernel:

$$
\Phi(X, Y)=\int_{E^{2}} K_{h}^{W e y l}(X, Y, Z) H(Z) d \mu_{E^{2}, h / 2}(Z) .
$$

By the change of variables $Z \rightarrow Z+(X+Y) / 2$, using (73) one obtains:

$$
\Phi(X, Y)=e^{\frac{1}{4 h}\left(|X|^{2}+|Y|^{2}\right)} \int_{E^{2}} e^{\frac{i}{h} \psi(X, Y, Z)} H\left(Z+\frac{X+Y}{2}\right) d \mu_{E^{2}, h / 2}(Z)
$$

where $\psi(X, Y, Z)$ is defined by

$$
\psi(X, Y, Z)=\frac{1}{2} \sigma(X, Y)-\sigma(Z, X-Y)
$$

Integrating by parts, we find an upper bound for this integral. For every $X=(x, \xi)$ in $E^{2}$, set:

$$
K_{E}(X)=\prod_{j \in I}\left(1+\frac{x_{j}^{2}}{h}\right)\left(1+\frac{x_{j}^{2}}{h}\right) \quad x_{j}=e_{j}(x)=u_{j}(X) \quad \xi_{j}=e_{j}(\xi)=u_{j}(X) .
$$

Integrations by parts then show that, for all $X$ and $Y$ in $E^{2}$ :

$$
K_{E}(X-Y)|\Phi(X, Y)| \leq e^{\frac{1}{4 h}\left(|X|^{2}+|Y|^{2}\right)} \int_{E^{2}} \mid\left(L H\left(Z+\frac{X+Y}{2}\right) \mid d \mu_{E^{2}, h / 2}(Z)\right.
$$

where $L$ is the differential operator defined by:

$$
L=\prod_{j \in I} L_{z_{j}} L_{\zeta_{j}} \quad L_{z_{j}}=\sum_{k=0}^{2} a_{k}\left(\frac{z_{j}}{\sqrt{h}}\right) h^{k / 2} \partial_{u_{j}}^{k}
$$

where

$$
a_{0}(z)=3-4 z^{2} \quad a_{1}(z)=4 z \quad a_{2}(z)=-1 .
$$

We then deduce that:

$$
K_{E}(X-Y)|\Phi(X, Y)| \leq e^{\frac{1}{4 h}\left(|X|^{2}+|Y|^{2}\right)} \sum_{(\alpha, \beta) \in \mathcal{M}_{2}(I)} h^{(|\alpha|+|\beta|) / 2}\left\|\partial_{u}^{\alpha} \partial_{v}^{\beta} H\right\|_{\infty} \prod_{j \in I} C_{\alpha_{j}} C_{\beta_{j}}
$$

with:

$$
C_{k}=\pi^{-1 / 2} \int_{\mathbb{R}}\left|a_{k}(z)\right| e^{-z^{2}} d z \quad k=0,1,2 .
$$

Notice that $\max \left(C_{0}, C_{1}, C_{2}\right) \leq 3$. Therefore:

$$
|\Phi(X, Y)| \leq e^{\frac{1}{4 h}\left(|X|^{2}+|Y|^{2}\right)} 9^{|I|} N_{I, h}^{(2)}(H) K_{E}(X-Y)^{-1} .
$$

We have:

$$
\int_{E^{2}} K_{E}(X)^{-1} d \lambda(X)=\left(h \pi^{2}\right)^{\operatorname{dim}(E)}
$$

Hence,

$$
|Q(U, V)| \leq(2 \pi h)^{-2 \operatorname{dim}(E)} 9^{|I|} N_{I, h}^{(2)}(H) \int_{E^{4}} K_{E}(X-Y)^{-1} e^{-\frac{1}{4 h}\left(|X|^{2}+|Y|^{2}\right)} U(X) V(Y) d \lambda(X, Y) .
$$

Using Schur's lemma and (94), one obtains (92) and (93). The proof of the proposition is then completed.

The first step of the proof of Proposition 3.1 is the following result: 
Proposition 5.2. Let $G$ be a function on $H^{2}$ and $I$ be a finite subset of $\Gamma$, such that $N_{I, h}^{(2)}(G)$ is well defined. We suppose that $G$ has a stochastic extension $\widetilde{G}$ (for the measure $\mu_{B^{2}, h}$ ). Let $E=E(I)$ be the subspace spanned by the $e_{j}(j \in I)$. Then, there exists a bounded operator $O p_{h}^{h y b, E(I)}(\widetilde{G})$ in $L^{2}\left(B, \mu_{B, h / 2}\right)$ such that, for all $f$ and g in $\mathcal{D}$ :

$$
Q_{h}^{h y b, E(I)}(\widetilde{G})(f, g)=<O p_{h}^{h y b, E(I)}(\widetilde{G}) f, g>
$$

Moreover, we have:

$$
\left\|O p_{h}^{h y b, E(I)}(\widetilde{G})\right\|_{\mathcal{L}\left(L^{2}\left(B, \mu_{B h / 2}\right)\right)} \leq\left(\frac{9 \pi}{2}\right)^{|I|} N_{I, h}^{(2)}(G)
$$

Proof. We use the expression for the quadratic form given by Proposition 4.11. Let $D \in \mathcal{F}\left(B^{\prime}\right)$ be a subspace such that $f$ and $g$ are in $\mathcal{D}_{D}$. Since the quadratic form defined in (87) does not depend on $D$, we may assume that $D$ contains $E=E(I)$. Let $S$ be the orthogonal of $E$ in $D$. The variable of $B^{2}$ may be written $Z=\left(Z_{E}, Z_{S}, Z_{D^{\perp}}\right)$ with $Z_{E^{\perp}}=\left(Z_{S}, Z_{D^{\perp}}\right)$. Let $\widehat{f}$ and $\widehat{g}$ be in $\mathcal{S}_{D}$ such that $f=\widehat{f} \circ P_{D}$ and $g=\widehat{g} \circ P_{D}$. The Segal Bargmann transforms $\widehat{T}_{h} \widehat{f}$ and $\widehat{T}_{h} \widehat{g}$ are functions of $Z_{D}=\left(Z_{E}, Z_{S}\right)$. With these notations, the equality (87) may be written:

$$
\begin{aligned}
Q_{h}^{h y b, E(I)}(\widetilde{G})(f, g)= & \int_{E^{4} \times S^{4} \times B^{2}} K_{h}^{W e y l}\left(X_{E}, Y_{E}, Z_{E}\right) K_{h}^{A W}\left(X_{S}, Y_{S}, Z_{S}\right) \\
& \left(\widehat{T}_{h} \widehat{f}\right)\left(X_{E}, X_{S}\right)\left(\widehat{T}_{h} \widehat{g}\right)\left(Y_{E}, Y_{S}\right) \widetilde{G}\left(Z_{E}, Z_{S}, Z_{D^{\perp}}\right) \\
& d \mu_{E^{4}, h}\left(X_{E}, Y_{E}\right) d \mu_{S^{4}, h}\left(X_{S}, Y_{S}\right) d \mu_{E^{2}, h / 2}\left(Z_{E}\right) d \mu_{S^{2}, h}\left(Z_{S}\right) d \mu_{\left(D^{\perp}\right)^{2}, h}\left(Z_{D^{\perp}}\right) .
\end{aligned}
$$

One applies Proposition 4.6 with $E_{1}$ replaced with $S$ and $E_{2}$ with $E$. This allows to write:

$$
\begin{aligned}
Q_{h}^{h y b, E(I)}(\widetilde{G})(f, g) & =\int_{E^{4} \times B^{2}} K_{h}^{W e y l}\left(X_{E}, Y_{E}, Z_{E}\right)\left(\widehat{T}_{h} \widehat{f}\right)\left(X_{E}, Z_{S}\right) \overline{\left(\widehat{T}_{h} \widehat{g}\right)\left(Y_{E}, Z_{S}\right)} \widetilde{G}\left(Z_{E}, Z_{S}, Z_{D^{\perp}}\right) \\
& d \mu_{E^{4}, h}\left(X_{E}, Y_{E}\right) d \mu_{E^{2}, h / 2}\left(Z_{E}\right) d \mu_{S^{2}, h}\left(Z_{S}\right) d \mu_{\left(D^{\perp}\right)^{2}, h}\left(Z_{D^{\perp}}\right)
\end{aligned}
$$

Let $\left(\Lambda_{n}\right)$ be an increasing sequence of finite subsets of $\Gamma$, containing $I$ and whose union is $\Gamma$. Since $\widetilde{G}$ is a stochastic extension of $G$ in the sense of Definition 4.4, one remarks that

$$
\lim _{n \rightarrow \infty}\left\|G \circ P_{E\left(\Lambda_{n}\right)}-\widetilde{G}\right\|_{L^{2}\left(E^{2} \times\left(E^{\perp}\right)^{2}, \mu_{E^{2}, h / 2} \otimes \mu_{\left(E^{\perp}\right)^{2}, h}\right)}=0 .
$$

Hence, for a subsequence, $G \circ P_{E\left(\Lambda_{\varphi(n)}\right)}$ converges to $\widetilde{G} \mu_{E^{2}, h / 2} \otimes \mu_{\left(E^{\perp}\right)^{2}, h^{-a l m o s t} \text { everywhere. Since } X \mapsto<}$ $\gamma_{D, h / 2} \widehat{f}, \Psi_{X, h}^{D, L e b}>_{L e b}$ and $X \mapsto<\gamma_{D, h / 2} \widehat{g}, \Psi_{X, h}^{D, L e b}>_{L e b}$ are rapidly decreasing one can write, using the dominated convergence Theorem,

$$
\begin{aligned}
Q_{h}^{h y b, E(I)}(\widetilde{G})(f, g) & =\lim _{n \rightarrow \infty} \int_{E^{4} \times B^{2}} K_{h}^{W e y l}\left(X_{E}, Y_{E}, Z_{E}\right)\left(\widehat{T}_{h} \widehat{f}\right)\left(X_{E}, Z_{S}\right) \overline{\left(\widehat{T}_{h} \widehat{g}\right)\left(Y_{E}, Z_{S}\right)} \\
& \left(G \circ P_{E\left(\Lambda_{\varphi(n)}\right)}\left(Z_{E}, Z_{S}, Z_{D^{\perp}}\right) d \mu_{E^{4}, h}\left(X_{E}, Y_{E}\right) d \mu_{E^{2}, h / 2}\left(Z_{E}\right) d \mu_{S^{2}, h}\left(Z_{S}\right) d \mu_{\left(D^{\perp}\right)^{2}, h}\left(Z_{D^{\perp}}\right) .\right.
\end{aligned}
$$

For all $n$, all $Z_{S}$ in $S^{2}$ and $Z_{D^{\perp}}$ in $\left(D^{\perp}\right)^{2}$, one defines a continuous function on $E^{2}$ by

$$
H_{n, Z_{S}, Z_{D^{\perp}}}\left(Z_{E}\right)=\left(G \circ P_{E\left(\Lambda_{n}\right)}\left(Z_{E}, Z_{S}, Z_{D^{\perp}}\right) .\right.
$$

The norm $N_{I, h}^{(2)}$ of this function is well defined by (91), the supremum being taken on $E$ and satisfies

$$
N_{I, h}^{(2)}\left(H_{n, Z_{S}, Z_{D^{\perp}}}\right) \leq N_{I, h}^{(2)}(G) .
$$

One can, then, apply Proposition 5.1, $H$ being replaced with $H_{n, Z_{S}, Z_{D \perp}}$ and $U\left(X_{E}\right)=\left(\widehat{T}_{h} \widehat{f}\right)\left(X_{E}, Z_{S}\right), V\left(X_{E}\right)=$ $\left(\widehat{T}_{h} \widehat{g}\right)\left(Y_{E}, Z_{S}\right)$. Using Proposition 5.1, Cauchy Schwarz's inequality and (97), one obtains, taking the limit on $n$,

$$
Q_{h}^{h y b, E(I)}(\widetilde{G})(f, g) \leq\left(\frac{9 \pi}{2}\right)^{|I|} N_{I, h}^{(2)}(G) A(f) A(g)
$$


where:

$$
A(f)^{2}=\int_{E^{2} \times S^{2}}\left|\left(\widehat{T}_{h} \widehat{f}\right)\left(X_{E}, Z_{S}\right)\right|^{2} d \mu_{E^{2}, h}\left(X_{E}\right) d \mu_{S^{2}, h}\left(Z_{S}\right) .
$$

According to (66), one has $A(f)=\|f\|_{L^{2}\left(B, \mu_{B, h / 2}\right)}$. Hence,

$$
Q_{h}^{h y b, E(I)}(\widetilde{G})(f, g) \leq\left(\frac{9 \pi}{2}\right)^{|I|} N_{I, h}^{(2)}(G)\|f\|_{L^{2}\left(B, \mu_{B, h / 2}\right)}\|g\|_{L^{2}\left(B, \mu_{B, h / 2}\right)} .
$$

The proposition is thus easily deduced.

We next involve the operator $T_{I, h}$ defined in (32). Proposition 3.1 will then follow from Proposition [5.2, applied with $G=T_{I, h} F$ combined with Proposition 5.3 below, applied to $F$. Indeed, Proposition 4.14 shows that the stochastic extension of $G$ for the measure $\mu_{B^{2}, h}$ is $\widetilde{G}=\widetilde{T}_{I, h} \widetilde{F}$.

Proposition 5.3. For any $F$ in $S_{2}(M, \varepsilon)$, for every finite subset $I$ of $\Gamma$ and for all $h>0$, the function $T_{I, h} F$ defined after (32) satisfies:

$$
N_{I, h}^{(2)}\left(T_{I, h} F\right) \leq M\left(18 S_{\varepsilon} h\right)^{|I|} \prod_{j \in I} \varepsilon_{j}^{2}
$$

Setting $u_{j}=\left(e_{j}, 0\right)$ and $v_{j}=\left(0, e_{j}\right)$, we write $\Delta_{j}=\partial_{u_{j}}^{2}+\partial_{v_{j}}^{2}$, and we consider the operator $H_{D_{j}, h / 2}$ defined as in (28), but acting on the functions on $H^{2}$. The proof of Proposition 5.3 will rely on the next lemma.

Lemma 5.4. If $H_{D_{j}, h / 2}$ is defined in (26), with $E$ replaced by $D_{j}=\operatorname{Vect}\left(e_{j}\right)$, we may write:

$$
I-H_{D_{j}, h / 2}=A_{j}=B_{j} \partial_{u_{j}}+C_{j} \partial_{v_{j}}=\frac{h}{4} V_{j} \Delta_{j}
$$

where the operators $B_{j}, C_{j}$ and $V_{j}$ are bounded in the space $C_{b}$ of continuous bounded functions on $H^{2}$. Moreover:

$$
\left\|A_{j}\right\|_{\mathcal{L}\left(C_{b}\right)} \leq 2 \quad\left\|B_{j}\right\|_{\mathcal{L}\left(C_{b}\right)} \leq(h / 2)^{1 / 2} \quad\left\|C_{j}\right\|_{\mathcal{L}\left(C_{b}\right)} \leq(h / 2)^{1 / 2} \quad\left\|V_{j}\right\|_{\mathcal{L}\left(C_{b}\right)} \leq 1 .
$$

Proof. The first estimate in (100) is standard. From the heat kernel's explicit expression, we get the first equality in (99) with:

$$
\begin{aligned}
& \left(B_{j} \varphi\right)(x, \xi)=-(\pi h)^{-1} \int_{\mathbb{R}^{2} \times[0,1]} e^{-\frac{1}{h}\left(u^{2}+v^{2}\right)} u \varphi\left(x+\theta u e_{j}, \xi+\theta v e_{j}\right) d u d v d \theta \\
& \left(C_{j} \varphi\right)(x, \xi)=-(\pi h)^{-1} \int_{\mathbb{R}^{2} \times[0,1]} e^{-\frac{1}{h}\left(u^{2}+v^{2}\right)} v \varphi\left(x+\theta u e_{j}, \xi+\theta v e_{j}\right) d u d v d \theta \\
& \left(V_{j} \varphi\right)(x, \xi)=-(\pi h)^{-1} \int_{\mathbb{R}^{2} \times[0,1]} e^{-\frac{1}{h}\left(u^{2}+v^{2}\right)} 2 \theta \varphi\left(x+\theta u e_{j}, \xi+\theta v e_{j}\right) d u d v d \theta
\end{aligned}
$$

We then deduce the norm estimates of $C_{j}$ and $V_{j}$ in (100). The last equality in (99) and the bound of $V_{j}$ in (100) are obtained using integrations by parts in (101) and (102).

Proof of Proposition 5.3. For any multi-index $(\alpha, \beta)$ in $\mathcal{M}_{2}(I)$, one may rewrite the operator $\partial_{u}^{\alpha} \partial_{v}^{\beta} T_{I, h}$ under the following form :

$$
\partial_{u}^{\alpha} \partial_{v}^{\beta} T_{I, h}=\prod_{j \in I} U_{j} \partial_{u_{j}}^{\alpha_{j}} \partial_{v_{j}}^{\beta_{j}}
$$

with:

$$
U_{j}=\left\{\begin{array}{lll}
A_{j} & \text { if } & \alpha_{j}+\beta_{j} \geq 2 \\
B_{j} \partial_{u_{j}}+C_{j} \partial_{v_{j}} & \text { if } & \alpha_{j}+\beta_{j}=1 \\
\frac{h}{4} V_{j} \Delta_{j} & \text { if } & \alpha_{j}+\beta_{j}=0
\end{array} .\right.
$$

Given the norm estimates for the operators $A_{j}, \ldots, D_{j}$ obtained in Lemma [5.4 we then deduce that, if $F$ belongs to $S_{2}(M, \varepsilon)$ :

$$
h^{(|\alpha|+|\beta|) / 2}\left\|\partial_{u}^{\alpha} \partial_{v}^{\beta} T_{I, h} F\right\|_{\infty} \leq M\left(2 h S_{\varepsilon}\right)^{|I|} \prod_{j \in I} \varepsilon_{j}^{2} .
$$

Since the number of elements of $\mathcal{M}_{2}(I)$ is $9^{|I|}$, we have indeed proved Proposition 5.3 


\section{Proof of Proposition 3.3.}

Proposition 3.3 will follow from Lemma 6.1 stated below, as will be proved at the end of the section. The proof of Lemma 6.1 will use Lemma 6.2 .

Lemma 6.1. Let $F$ be in $S_{2}(M, \varepsilon)$, where the sequence $\left(\varepsilon_{j}\right)_{(j \in \Gamma)}$ is square summable. Let $h$ be positive. Let $\widetilde{F}$ be a function on $B^{2}$ which is the stochastic extension of $F$ both for the measure $\mu_{B^{2}, h}$ and for the measure $\mu_{B^{2}, h / 2}$. Then, setting $E_{n}=E\left(\Lambda_{n}\right)$, and using the operators $\widetilde{H}_{E_{n}^{\perp}, h / 2}$ defined in 27):

$$
\left\|\widetilde{H}_{E_{n}^{\perp}, h / 2} \widetilde{F}-\widetilde{F}\right\|_{L^{1}\left(B^{2}, \mu_{B^{2}, h / 2}\right)} \leq K M\left[\sum_{\lambda_{j} \notin \Lambda_{n}} \varepsilon_{j}^{2}\right]^{1 / 2}+K M \sum_{\lambda_{j} \notin \Lambda_{n}} \varepsilon_{j}^{2} .
$$

Proof. We have $\widetilde{H}_{E_{n}^{\perp}, h / 2}\left(F \circ P_{E_{n}}\right)=F \circ P_{E_{n}}$ and consequently:

$$
\left\|\widetilde{H}_{E_{n}^{\perp}, h / 2} \widetilde{F}-\widetilde{F}\right\|_{L^{1}\left(B^{2}, \mu_{B^{2}, h / 2}\right)} \leq\left\|\widetilde{F}-F \circ P_{E_{n}}\right\|_{L^{1}\left(B^{2}, \mu_{B^{2}, h / 2}\right)}+\left\|\widetilde{H}_{E_{n}^{\perp}, h / 2}\left(\widetilde{F}-F \circ P_{E_{n}}\right)\right\|_{L^{1}\left(B^{2}, \mu_{B^{2}, h / 2}\right)}
$$

One denotes by $\nu_{n h}$ the measure $\nu_{B^{2}, E_{n}^{2}, h / 2, h}$ defined by (53). According to Proposition 4.5, the operator $\widetilde{H}_{E_{n}^{\perp}, h / 2}$ is bounded from $L^{1}\left(B^{2}, \nu_{n, h}\right)$ into $L^{1}\left(B^{2}, \mu_{B^{2}, h / 2}\right)$, with a norm $\leq 1$. Consequently, since the injection of $L^{2}\left(B^{2}, \nu_{n, h}\right)$ in $L^{1}\left(B^{2}, \nu_{n, h}\right)$ has norm 1 :

$$
\left\|\widetilde{H}_{E_{n}^{\perp}, h / 2} \widetilde{F}-\widetilde{F}\right\|_{L^{1}\left(B^{2}, \mu_{B^{2}, h / 2}\right)} \leq\left\|\widetilde{F}-F \circ P_{E_{n}}\right\|_{L^{2}\left(B^{2}, \mu_{B^{2}, h / 2}\right)}+\left\|\widetilde{F}-F \circ P_{E_{n}}\right\|_{L^{2}\left(B^{2}, \nu_{n, h}\right)} .
$$

From Lemma 6.2. we have, for $m>n$ :

$$
\begin{aligned}
& \left\|\left(F \circ P_{E_{m}}\right)-\left(F \circ P_{E_{n}}\right)\right\|_{L^{2}\left(B^{2}, \mu_{B^{2}, h}\right)}+\left\|\left(F \circ P_{E_{m}}\right)-\left(F \circ P_{E_{n}}\right)\right\|_{L^{2}\left(B^{2}, \nu_{n, h}\right)} \\
& \leq C M\left[\sum_{j \notin \Lambda_{n}} \varepsilon_{j}^{2}\right]^{1 / 2}+C M \sum_{j \notin \Lambda_{n}} \varepsilon_{j}^{2}
\end{aligned}
$$

where $C$ is a constant which depends only on $h$. Since $\widetilde{F}$ is the stochastic extension of $F$ for the measure $\mu_{B^{2}, h}$ and since $F$ is bounded, we deduce that the sequence $F \circ P_{E_{m}}$ tends to $\widetilde{F}$ in $L^{2}\left(B^{2}, \mu_{B^{2}, h}\right)$. For all $n$ fixed, the measures $\nu_{n, h}=\mu_{E_{n}^{2}, h / 2} \otimes \mu_{\left(E_{n}^{\perp}\right)^{2}, h}$ and $\mu_{B^{2}, h}=\mu_{E_{n}^{2}, h} \otimes \mu_{\left(E_{n}^{\perp}\right)^{2}, h}$ are equivalent as a product of equivalent measures (since $E_{n}$ is finite dimensional). Then, the convergence in probability for one of them is equivalent to the convergence in probability for the other one. Since $\widetilde{F}$ is the stochastic extension of $F$ for the measure $\mu_{B^{2}, h / 2}$, we thus deduce that, for all $n$ fixed, the sequence $F \circ P_{E_{m}}$ tends $\widetilde{F}$ in probability for the measure $\nu_{n, h}$ when $m$ tends to infinity. Consequently, this sequence tends to $\widetilde{F}$ in $L^{2}\left(B^{2}, \nu_{n, h}\right)$ for $n$ fixed, $m$ going to infinity. In particular:

$$
\begin{aligned}
& \left\|\widetilde{F}-F \circ P_{E_{n}}\right\|_{L^{2}\left(B^{2}, \mu_{B^{2}, h / 2}\right)}+\left\|\widetilde{F}-F \circ P_{E_{n}}\right\|_{L^{2}\left(B^{2}, \nu_{n, h}\right)} \leq \\
& \leq C M\left[\sum_{j \notin \Lambda_{n}} \varepsilon_{j}^{2}\right]^{1 / 2}+C M \sum_{j \notin \Lambda_{n}} \varepsilon_{j}^{2} .
\end{aligned}
$$

The Lemma is then deduced from (105) and (107).

Lemma 6.2. With the notations and assumptions of Lemma 6.1, Inequality (106) holds true, with a constant $C>0$ depending only on $h$.

Proof. Let $\Lambda_{m} \backslash \Lambda_{n}=\left\{\alpha_{1}, \ldots, \alpha_{p}\right\}$, with $\alpha_{j} \in \Gamma$. Set $I_{0}=\Lambda_{n}$ and $I_{j}=\Lambda_{n} \cup\left\{e_{\alpha_{1}}, \ldots, e_{\alpha_{j}}\right\}$. One may write, from an order 2 Taylor formula, and since $F$ belongs to $S(M, \varepsilon)$ :

$$
\left(F \circ P_{E\left(I_{j}\right)}-\left(F \circ P_{E\left(I_{j-1}\right)}\right)=\varphi_{j}+\psi_{j}\right.
$$


with

$$
\begin{gathered}
\varphi_{j}(x, \xi)=\left(\partial_{u_{\alpha_{j}}} F\right)\left(P_{E\left(I_{j-1}\right)}(x, \xi)<e_{\alpha_{j}}, x>+\left(\partial_{v_{\alpha_{j}}} F\right)\left(P_{E\left(I_{j-1}\right)}(x, \xi)<e_{\alpha_{j}}, \xi>,\right.\right. \\
\psi_{j}(x, \xi)=a_{j}(x, \xi)<e_{\alpha_{j}}, x>^{2}+b_{j}(x, \xi)<e_{\alpha_{j}}, x><e_{\alpha_{j}}, \xi>+c_{j}(x, \xi)<e_{\alpha_{j}}, \xi>^{2}, \\
\mid\left(\partial_{u_{\alpha_{j}}} F\right)\left(P _ { E ( I _ { j - 1 } ) } ( x , \xi ) | + | ( \partial _ { v _ { \alpha _ { j } } } F ) \left(P_{E\left(I_{j-1}\right)}(x, \xi) \mid \leq 2 M \varepsilon_{j},\right.\right. \\
\left|a_{j}(x, \xi)\right|+\left|b_{j}(x, \xi)\right|+\left|c_{j}(x, \xi)\right| \leq C M \varepsilon_{j}^{2} .
\end{gathered}
$$

We then deduce that, with a constant $C$ depending only on $h$ :

$$
\begin{gathered}
\left\|\varphi_{j}\right\|_{L^{2}\left(B^{2}, \mu_{B^{2}, h}\right)}+\left\|\varphi_{j}\right\|_{L^{2}\left(B^{2}, \nu_{n, h}\right)} \leq C M \varepsilon_{\alpha_{j}}, \\
\left\|\psi_{j}\right\|_{L^{2}\left(B^{2}, \mu_{B^{2}, h}\right)}+\left\|\psi_{j}\right\|_{L^{2}\left(B^{2}, \nu_{n, h}\right)} \leq C M \varepsilon_{\alpha_{j}}^{2} .
\end{gathered}
$$

Let us show that, when $j>k$, the functions $\varphi_{j}$ and $\varphi_{k}$ are orthogonal, both in $L^{2}\left(B^{2}, \mu_{B^{2}, h}\right)$ and in $L^{2}\left(B^{2}, \nu_{n, h}\right)$. Indeed, one may write, for $j>k$, with some suitable functions $U_{j k}$ and $V_{j k}$ :

$$
<\varphi_{j}, \varphi_{k}>_{L^{2}\left(B^{2}, \mu_{B^{2}, h}\right)}=\int_{B^{2}}\left[U_{j k}\left(P_{E_{j-1}}(X)\right)<u_{\alpha_{j}}, X>+V_{j k}\left(P_{E_{j-1}}(X)\right)<v_{\alpha_{j}}, X>\right] d \mu_{B^{2}, h}(X)
$$

and the same holds true for the measure $\nu_{n h}$. However, from (8), the integral of the above cylinder function is vanishing for the measure $\mu_{B^{2}, h}$ and also for the measure $\nu_{n, h}$. Thus, $\varphi_{j}$ and $\varphi_{k}$ are orthogonal if $j>k$. Then, one has:

$$
\left(F \circ P_{E\left(\Lambda_{m}\right.}\right)-\left(F \circ P_{E\left(\Lambda_{n}\right)}\right)=\sum_{j=1}^{p}\left(\varphi_{j}+\psi_{j}\right)
$$

and, from the orthogonality property:

$$
\left\|\left(F \circ P_{E_{m}}\right)-\left(F \circ P_{E_{n}}\right)\right\|_{L^{2}\left(B^{2}, \mu_{B^{2}, h}\right)} \leq\left[\sum_{j=1}^{p}\left\|\varphi_{j}\right\|_{L^{2}\left(B^{2}, \mu_{B^{2}, h}\right)}^{2}\right]^{1 / 2}+\sum_{j=1}^{p}\left\|\psi_{j}\right\|_{L^{2}\left(B^{2}, \mu_{B^{2}, h}\right)}
$$

and this is also similarly valid for the measure $\nu_{n, h}$. We then deduce (106). The proof of the Lemma is finished.

End of the proof of Proposition 3.3. By Proposition 4.10, (with $m=0$ ), we have, for all $f$ and $g$ in $\mathcal{D}_{D}$ $\left(D \in \mathcal{F}\left(B^{\prime}\right)\right)$ :

$$
\left|Q_{h}^{W e y l}\left(\widetilde{H}_{E_{\frac{1}{n}}^{\perp}, h / 2} \widetilde{F}\right)(f, g)-Q_{h}^{W e y l}(\widetilde{F})(f, g)\right| \leq I_{D, 0, h}(f) I_{D, 0, h}(g) N_{0}\left(H_{E_{n}^{\perp}, h / 2} \widetilde{F}-\widetilde{F}\right) .
$$

For all $Y$ in $H^{2}$ and for every function $F$ on $B^{2}$ (resp. on $H^{2}$ ), let us denote by $\tau_{Y}$ the function $X \rightarrow F(X+Y)$ on $B^{2}$ (resp. on $H^{2}$ ). According to (12):

$$
\begin{aligned}
N_{0}\left(H_{E_{n}^{\perp}, h / 2} \widetilde{F}-\widetilde{F}\right) & =\sup _{Y \in H^{2}}\left\|\tau_{Y}\left(H_{E_{n}^{\perp}, h / 2} \widetilde{F}-\widetilde{F}\right)\right\|_{L^{1}\left(B^{2}, \mu_{B^{2}, h / 2}\right)} \\
& =\sup _{Y \in H^{2}}\left\|H_{E_{n}^{\perp}, h / 2}\left(\tau_{Y} \widetilde{F}\right)-\left(\tau_{Y} \widetilde{F}\right)\right\|_{L^{1}\left(B^{2}, \mu_{B^{2}, h / 2}\right.} .
\end{aligned}
$$

One then applies Proposition 6.1, replacing $F$ with $\tau_{Y} F$, for all $Y$ in $H^{2}$. Since $F$ is in $S_{2}(M, \varepsilon)$ too, since the sequence $\left(\varepsilon_{j}\right)$ is square summable, inequality (103) proves that the right term of (108) converges to 0 when $n$ goes to infinity. This proves Proposition 3.3 . 


\section{$7 \quad$ Wick symbol of a Weyl operator}

For all $X$ in $H^{2}$, let $\Psi_{X h}$ be the coherent state defined by (58) as a function on $H$ and $\widetilde{\Psi}_{X h}$ its stochastic extension, defined almost everywhere on $B$ by (59). For every operator $A$, bounded on $L^{2}\left(B, \mu_{B, h / 2}\right)$, one can define the Wick symbol of $A$ by

$$
\sigma_{h}^{W i c k}(A)(X)=<A \widetilde{\Psi}_{X h}, \widetilde{\Psi}_{X h}>.
$$

See Berezin $[\mathrm{Be}]$ or Folland $[\mathrm{F}]$.

Theorem 7.1. Let $F$ be a function in $S_{2}(M, \varepsilon)$, admitting a stochastic extension $\widetilde{F}$ (Definition 4.4, second point, $p=2$ ). One denotes by $Q_{h}^{W e y l}(\widetilde{F})$ the quadratic form associated with $\widetilde{F}$ by Definition 1.2 and $O p_{h}^{\text {Weyl }}(F)$ the bounded extension whose existence results from Theorem 1.4. Then one has, for all $X$ in $H^{2}$ :

$$
\sigma_{h}^{W i c k}\left(O p_{h}^{W e y l}(F)\right)(X)=\int_{B^{2}} \widetilde{F}(X+Y) d \mu_{B^{2}, h / 2}(Y) .
$$

According to Gross ( $\mathrm{G}-4$, , Prop.9) or Kuo ( $[\underline{\mathrm{K}}$, (Th.6.2, Chapter 2)) the integral (110) defines a continuous function on $H^{2}$.

Proof. We begin by proving that, for all $X$ and $Y$, in $H^{2}$ :

$$
<O p_{h}^{W e y l}(F)\left(\widetilde{\Psi}_{X, h}\right), \widetilde{\Psi}_{Y, h}>=\int_{B^{2}} \widetilde{F}(Z) e^{-\frac{1}{4 h}\left(|X|^{2}+|Y|^{2}\right)} \widetilde{K}_{h}^{W e y l}(X, Y, Z) d \mu_{B^{2}, h / 2}(Z) .
$$

Let $\left(E_{n}\right)$ be an increasing sequence in $\mathrm{s} \mathcal{F}\left(B^{\prime}\right)$, whose union is dense in $H$. According to Definition 1.2, one has, for all $n \geq 1$ and all $X$ and $Y$ in $H$ :

$$
<O p_{h}^{W e y l}(\widetilde{F})\left(\Psi_{X, h} \circ P_{E_{n}}\right), \Psi_{Y, h} \circ P_{E_{n}}>=\int_{B^{2}} \widetilde{F}(Z) H_{h}^{\text {Gauss }}\left(\Psi_{X, h} \circ P_{E_{n}}, \Psi_{Y, h} \circ P_{E_{n}}\right)(Z) d \mu_{B^{2}, h / 2}(Z) .
$$

When $n$ goes to infinity one has, by definition of the stochastic extension:

$$
\lim _{n \rightarrow+\infty} \Psi_{X, h} \circ P_{E_{n}}=\widetilde{\Psi}_{X, h}
$$

The convergence is in $L^{2}\left(B, \mu_{B, h / 2}\right)$. Since $O p_{h}^{W e y l}(F)$ is a bounded extension, one deduces that:

$$
<O p_{h}^{\text {Weyl }}(F)\left(\widetilde{\Psi}_{X, h}\right), \widetilde{\Psi}_{Y, h}>=\lim _{n \rightarrow+\infty}<O p_{h}^{W e y l}(\widetilde{F})\left(\Psi_{X, h} \circ P_{E_{n}}\right), \Psi_{Y, h} \circ P_{E_{n}}>.
$$

According to Proposition 4.9, one has

$$
\begin{aligned}
& \lim _{n \rightarrow+\infty} \int_{B^{2}} \widetilde{F}(Z) H_{h}^{\text {Gauss }}\left(\Psi_{X, h} \circ P_{E_{n}}, \Psi_{Y, h} \circ P_{E_{n}}\right)(Z) d \mu_{B^{2}, h / 2}(Z)= \\
& =\int_{B^{2}} \widetilde{F}(Z) e^{-\frac{1}{4 h}\left(|X|^{2}+|Y|^{2}\right)} \widetilde{K}_{h}^{W e y l}(X, Y, Z) d \mu_{B^{2}, h / 2}(Z),
\end{aligned}
$$

which gives (111). Restricting (111) to $Y=X$ and using (77), one gets:

$$
\sigma_{h}^{W i c k}\left(O p_{h}^{W e y l}(F)\right)(X)=\int_{B^{2}} \widetilde{F}(Z) e^{\frac{2}{h} \ell_{X}(Z)-\frac{1}{h}|X|^{2}} d \mu_{B^{2}, h / 2}(Z) .
$$

According to Proposition 4.2, where $B$ is replaced with par $B^{2}, h$ with $h / 2$ and $a$ with $X$, one indeed obtains (110).

\section{Examples}

We shall give examples of Wiener spaces, of stochastic extensions and, finally, of functions $F$ in $S_{m}(M, \varepsilon)$. 


\subsection{Examples of Wiener spaces}

We begin by recalling how, starting from a real separable infinite-dimensional Hilbert space $H$, one can always construct a Banach space $B$ and an injection $i$ such that $(i, H, B)$ is an abstract Wiener space. This construction is due to Gross ([G-3], example 2 p. 92)). Then we recall two other classical examples of Wiener spaces, related to the Brownian motion or to the field theory. To conclude, we give a more original example, related to interacting lattices.

Example 8.1. Let $H$ be a real separable Hilbert space, with norm $|\cdot|$ and let $D$ be an injective HilbertSchmidt operator in $H$. Let $B$ be the completion of $H$ with respect to the norm $x \rightarrow|D x|$ and $i$ be the canonical injection from $H$ into $B$. Then $(i, H, B)$ is an abstract Wiener space.

Example 8.2. The classical Wiener space. In this case, $H$ is the Cameron-Martin space, that is, the space of all real valued functions in $H^{1}(0,1)$ vanishing at the origin. This space has the scalar product

$$
<u, v>=\int_{0}^{1} u^{\prime}(t) v^{\prime}(t) d t
$$

One can choose, for $B$, the space of all continuous functions on $[0,1]$ vanishing at the origin. This space is equipped with the norm

$$
\|f\|_{B}=\sup _{t \in[0,1]}|f(t)| .
$$

One proves (cf Kuo $[\mathrm{K}]$, Chapter 1, Section 5) that, if $i$ is the natural injection from $H$ into $B$, the triple $(i, H, B)$ is an abstract Wiener space. The measure $\mu_{B, h}$ on $B$ is the classical Wiener measure.

The following example is concerned with the free euclidean field of mass $m$, or free boson field, or free Markoff field, cf [SI]. The space $H_{m}$ below appears in [SI. Nevertheless, [SI does not use the Wiener spaces of Definition 4.1, but a Gaussian measure supported by $\mathcal{S}^{\prime}\left(\mathbb{R}^{2}\right)$. One finds in Reed-Rosen [R-R the following example, using the Wiener spaces of Definition 4.1.

Example 8.3. Let $H$ be the space of tempered distributions $f$ on $\mathbb{R}^{2}$ whose Fourier Transform $\widehat{f}$ is in $L^{2}$ locally and such that, for all $m>0$ :

$$
\|f\|_{H_{m}}^{2}=2 \int_{\mathbb{R}^{2}} \frac{|\widehat{f}(\xi)|^{2}}{m^{2}+|\xi|^{2}} d \lambda(\xi)<+\infty
$$

An example of a Banach space $B$ such that $(i, H, B)$ is a Wiener space is developed in [R-R. Let $B$ be the completion of $\mathcal{S}\left(\mathbb{R}^{2}\right)$ with respect to the following norm, where $\alpha>0$ and $\beta>1 / 2$ :

$$
\begin{gathered}
\|f\|_{B}=\left\|P_{1}^{-\alpha} Q_{\beta}^{-1} P^{-1} f\right\|_{L^{2}}, \\
P=(1-\Delta)^{1 / 2} \quad P_{1}=\left(1-\partial_{x_{1}}^{2}\right)^{1 / 2} \quad Q_{\beta}(x)=\left(1+|x|^{2}\right)^{1 / 2}\left[\ln \left(\left(1+|x|^{2}\right)^{1 / 2}\right)\right]^{\beta} .
\end{gathered}
$$

Let $i$ be the natural injection from $H$ in $B$. Then $(i, H, B)$ is an abstract Wiener space (cf $[\mathrm{R}-\mathrm{R}]$ ). Indeed, $P$ is an isomorphism from $L^{2}\left(\mathbb{R}^{2}\right)$ on $H$. Reed and Rosen prove (Lemma 1 ) that $P^{-1} P_{1}^{-\alpha} Q_{\beta}^{-1}$ is a Hilbert-Schmidt operator in $L^{2}\left(\mathbb{R}^{2}\right)$ if $\alpha>0$ and $\beta>1 / 2$. Hence $P\left(P^{-1} P_{1}^{-\alpha} Q_{\beta}^{-1}\right) P^{-1}$ is Hilbert-Schmidt in $H$. The statement of $[\mathrm{R}-\mathrm{R}]$ is, then, a consequence of example 8.1.

The following example shows that our results apply to interacting lattices. The Wiener space $(i, H, B)$ which is defined there exceeds the frame of Example 8.1.

Proposition 8.1. Let $H=\ell^{2}(\Gamma, \mathbb{R})$, where $\Gamma$ is a countable set, for example a lattice in $\mathbb{Z}^{d}$. Choose a family $b=\left(b_{j}\right)_{(j \in \Gamma)}$ of real positive numbers such that, for all $\varepsilon>0$, the family of positive real numbers

$$
R_{j}\left(b_{j}, \varepsilon\right)=\int_{\varepsilon b_{j}}^{+\infty} e^{-\frac{x^{2}}{2}} d x \quad j \in \Gamma
$$


is summable. Let $B$ denote the space of all families $\left(x_{j}\right)_{(j \in \Gamma)}$ such that $\left(\frac{\left|x_{j}\right|}{b_{j}}\right)_{(j \in \Gamma)}$ is bounded and converges to zero when $j$ goes to infinity. This space has the norm

$$
\|x\|_{B}=\sup _{j \in \Gamma} \frac{\left|x_{j}\right|}{b_{j}} .
$$

In this case, the space $H=\ell^{2}(\Gamma, \mathbb{R})$ is densely embedded in $B$. Moreover, for all $h>0$, the restriction to $H$ of the norm of $B$ is $H$-measurable.

Proof. A seminorm $N$ on a Hilbert space $H$ is said to be tame if it has the form $N(x)=\widetilde{N}\left(\pi_{E}(x)\right)$, where $E$ is a subspace in $\mathcal{F}(H), \pi_{E}: H \rightarrow E$ is the orthogonal projections on $E$ and $\widetilde{N}$ is a norm on $E$. One chooses an increasing sequence of finite subsets $\Gamma_{p}$ of $\Gamma$, whose union is $\Gamma$. One defines an increasing sequence $N_{p}$ of tame semi-norms on $H=\ell^{2}(\Gamma, \mathbb{R})$ by setting:

$$
N_{p}(x)=\sup _{j \in \Gamma_{p}} \frac{\left|x_{j}\right|}{b_{j}} .
$$

It is well known (cf. L. Gross G-3, Theorem 1, p. 95), that, if $N_{p}$ is an increasing sequence of tame semi-norms on $H$ and if, for all $h>0$ and $\varepsilon>0$,

$$
\lim _{p \rightarrow \infty} \mu_{H, h}\left(\left\{x \in H, \quad N_{p}(x) \leq \varepsilon\right\}\right)>0,
$$

then $\lim _{p \rightarrow \infty} N_{p}(x)$ exists for all $x \in H$ and the limit defines a $H$-measurable semi-norm (see Definition 4.1). For all $\varepsilon>0$, the set

$$
C_{p}=\left\{x \in H, \quad N_{p}(x) \leq \varepsilon\right\}
$$

is a cylinder set of $H$. It can be written $C_{p}=\pi_{E}^{-1}(\Omega)$, with $E=\mathbb{R}^{\Gamma_{p}}$ and $\Omega=\prod_{j \in \Gamma_{p}}\left[-\varepsilon b_{j}, \varepsilon b_{j}\right]$. Its $\mu_{H, h^{-}}$ measure, defined in (42), is therefore

$$
\mu_{H, h}\left(C_{p}\right)=\prod_{j \in \Gamma_{p}}(2 \pi h)^{-1 / 2} \int_{-\varepsilon b_{j}}^{\varepsilon b_{j}} e^{-\frac{x^{2}}{2 h}} d x
$$

The sequence $\mu_{H, h}\left(C_{p}\right)$ decreases to a nonnegative limit. One has

$$
\mu_{H, h}\left(C_{p}\right)=\prod_{j \in \Gamma_{p}}\left[1-2(2 \pi h)^{-1 / 2} \int_{\varepsilon b_{j}}^{+\infty} e^{-\frac{x^{2}}{2 h}} d x\right]=\prod_{j \in \Gamma_{p}}\left[1-2(2 \pi)^{-1 / 2} R_{j}\left(b_{j}, \frac{\varepsilon}{\sqrt{h}}\right)\right]
$$

where $R_{j}(.,$.$) is defined by (115). Since the factors of this product are positive, the limit is positive provided the$ family $R_{j}\left(b_{j}, \frac{\varepsilon}{\sqrt{h}}\right)$ is summable. Hence the sequence $\left(N_{p}\right)$ satisfies (118) and the limit of this sequence, which is the restriction of the norm of $B$ to $H$, is $H$ - measurable.

For example if $\Gamma=\mathbb{Z}^{d}$ and if $\gamma>0$, the family of real numbers $b_{j}=(1+|j|)^{\gamma}$ is such that the family $R_{j}\left(b_{j}, \varepsilon\right)$ defined in (115) is summable for all $\varepsilon>0$. Hence this family $\left(b_{j}\right)$ can be used in the definition of the Banach space before (116).

\subsection{Examples of stochastic extensions.}

The most natural example of a stochastic extension is the continuous extension, using density arguments. The following proposition is not very different from $[\mathrm{K}$, Chapter 1, Theorem 6.3.

Proposition 8.2. If $f$ is bounded on $H$ and uniformly continuous with respect to the restriction, to $H$, of the norm of $B$, then the stochastic extension and the extension of $f$ obtained by density are almost everywhere the same. 
Kree, Rączka and B. Lascar sometimes supposed the symbols were Fourier transforms of bounded measures on $H^{2}$. The following proposition shows that such functions admit stochastic extensions.

Proposition 8.3. Let $\nu$ be a complex bounded measure on the Borel $\sigma$-algebra of a real separable Hilbert space $H$. Let $(i, H, B)$ be an abstract Wiener space. For every $x$ in $H$, set

$$
\widehat{\nu}(x)=\int_{H} e^{i u \cdot x} d \nu(u) .
$$

Then for every positive $h$, the function $\widehat{\nu}$ has a stochastic extension for the measure $\mu_{B, h}$, in the sense of Definition 4.4.

Proof. Let $\left(E_{n}\right)$ be an increasing sequence of $\mathcal{F}(H)$, whose union is dense in $H$. Let $\pi_{n}: H \rightarrow E_{n}$ be the orthogonal projection on $E_{n}$ and $\widetilde{\pi}_{n}: B \rightarrow E_{n}$ its stochastic extension, defined by (50). If $m<n$, one has, using Fubini's theorem and Jensen's inequality:

$$
\left\|\widehat{\nu} \circ \widetilde{\pi}_{n}-\widehat{\nu} \circ \widetilde{\pi}_{m}\right\|_{L^{2}\left(B, \mu_{B, h}\right)}^{2} \leq|\nu|(H) \int_{H \times B}\left|e^{i u \cdot \widetilde{\pi}_{n}(x)}-e^{i u \cdot \widetilde{\pi}_{m}(x)}\right|^{2} d|\nu|(u) d \mu_{B, h}(x) .
$$

According to (49) and (51), for all $u$ in $H$ :

$$
\int_{B}\left|e^{i u \cdot \widetilde{\pi}_{m}(x)}-e^{i u \cdot \widetilde{\pi}_{n}(x)}\right|^{2} d \mu_{B, h}(x) \leq \inf \left(2, h\left|\pi_{m}(u)-\pi_{n}(u)\right|^{2}\right) .
$$

Hence

$$
\left\|\widehat{\nu} \circ \widetilde{\pi}_{n}-\widehat{\nu} \circ \widetilde{\pi}_{m}\right\|_{L^{2}\left(B, \mu_{B, h}\right)}^{2} \leq|\nu|(H) \int_{H} \inf \left(2, h\left|\pi_{n}(u)-\pi_{m}(u)\right|^{2}\right) d|\nu|(u) .
$$

According the dominated convergence Theorem, the sequence of functions $\widehat{\nu} \circ \widetilde{\pi}_{n}$ is a Cauchy sequence in $L^{2}\left(B, \mu_{B, h}\right)$. The same argument shows that the limit does not depend on the sequence of subspaces. Therefore, the function $\widehat{\nu}$ admits a stochastic extension in the sense of $L^{2}\left(B, \mu_{B, h}\right)$ (Definition 4.4).

We now prove that, if the sequence $\left(\varepsilon_{j}\right)$ is summable, Hypothesis (H1) of Theorem 1.4 implies Hypothesis (H2), that is the existence of a stochastic extension.

Proposition 8.4. Let $F$ be a function in $S_{1}(M, \varepsilon)$, with respect to a Hilbert basis $\left(e_{j}\right)_{(j \in \Gamma)}$, where the sequence $\left(\varepsilon_{j}\right)_{(j \in \Gamma)}$ is summable. Then, for every positive $h, F$ admits a stochastic extension in $L^{1}\left(B^{2}, \mu_{B^{2}, h}\right)$.

Proof. Up to a change of notations, one can suppose that $\Gamma$ is the set of integers greater than 1 . For all $j \geq 1$, set $u_{j}=\left(e_{j}, 0\right)$ and $v_{j}=\left(0, e_{j}\right)$. Let $F_{p}$ be the subspace spanned by the $u_{j}$ and $v_{j}(j \leq p)$. Let $\left(E_{n}\right)$ be an increasing sequence of $\mathcal{F}\left(H^{2}\right)$, whose union is dense in $H^{2}$. For all $m$ and $n$ such that $m<n$, let $S_{m n}$ be the orthogonal supplement of $E_{m}$ in $E_{n}$. We now prove that:

$$
\int_{B^{2}}\left|F\left(\widetilde{\pi}_{E_{m}}(X)\right)-F\left(\widetilde{\pi}_{E_{n}}(X)\right)\right| d \mu_{B^{2}, h}(X) \leq M \sum_{j=1}^{\infty} \varepsilon_{j}\left(\left|\pi_{S_{m n}}\left(u_{j}\right)\right|+\left|\pi_{S_{m n}}\left(v_{j}\right)\right|\right) .
$$

For all $p \geq 1$, for all $X$ and $Y$ in $H^{2}$ set:

$$
F_{p}(X, Y)=F\left(\pi_{F_{p}}(Y)+\pi_{\left(F_{p}\right)^{\perp}}(X)\right)-F(X) .
$$

Since $F$ is continuous, one has:

$$
\lim _{p \rightarrow \infty} F_{p}(X, Y)=F(Y)-F(X) \quad(X, Y) \in H^{4} .
$$

One can write

$$
F_{p}(X, Y)=\sum_{j=1}^{p} \varphi_{j}(X, Y) \quad \varphi_{j}(X, Y)=F\left(\pi_{F_{j}}(Y)+\pi_{\left(F_{j}\right)^{\perp}}(X)\right)-F\left(\pi_{F_{j-1}}(Y)+\pi_{\left(F_{j-1}\right)^{\perp}}(X)\right) .
$$


According to Taylor's formula, there exist functions $A_{j}(X, Y)$ and $B_{j}(X, Y)$ such that

$$
\varphi_{j}(X, Y)=A_{j}(X, Y) u_{j} \cdot(X-Y)+B_{j}(X, Y) v_{j} \cdot(X-Y) \quad\left|A_{j}(X, Y)\right| \leq M \varepsilon_{j} \quad\left|B_{j}(X, Y)\right| \leq M \varepsilon_{j} .
$$

Therefore,

$$
\left|F_{p}(X, Y)\right| \leq M \sum_{j=1}^{p} \varepsilon_{j}\left(\left|u_{j} \cdot(X-Y)\right|+\left|v_{j} \cdot(X-Y)\right|\right) .
$$

It follows that, if $m<n$, for almost every $X$ in $B^{2}$ :

$$
\left|F_{p}\left(\widetilde{\pi}_{E_{m}}(X), \widetilde{\pi}_{E_{n}}(X)\right)\right| \leq M \sum_{j=1}^{p} \varepsilon_{j}\left(\left|u_{j} \cdot \widetilde{\pi}_{S_{m n}}(X)\right|+\left|v_{j} \cdot \widetilde{\pi}_{S_{m n}}(X)\right|\right) .
$$

According to (51):

$$
\left|F_{p}\left(\widetilde{\pi}_{E_{m}}(X), \widetilde{\pi}_{E_{n}}(X)\right)\right| \leq M \sum_{j=1}^{p} \varepsilon_{j}\left(\left|\ell_{\pi_{S_{m n}}\left(u_{j}\right)}(X)\right|+\left|\ell_{\pi_{S_{m n}}\left(v_{j}\right)}(X)\right|\right) .
$$

According to (44), in $L^{1}\left(B^{2}, \mu_{B^{2}, h}\right)$ :

$$
\int_{B^{2}}\left|F_{p}\left(\widetilde{\pi}_{E_{m}}(X), \tilde{\pi}_{E_{n}}(X)\right)\right| d \mu_{B^{2}, h}(X) \leq M \sqrt{2 h / \pi} \sum_{j=1}^{p} \varepsilon_{j}\left(\left|\pi_{S_{m n}}\left(u_{j}\right)\right|+\left|\pi_{S_{m n}}\left(v_{j}\right)\right|\right) .
$$

Letting $p$ grow to infinity and using (121), one obtains (120). Since the sequence $\left(\varepsilon_{j}\right)_{(j \in \Gamma)}$ is summable, the right term of (120) converges to 0 when $m$ goes to infinity, according to the dominated convergence Theorem, which proves the proposition.

We now give examples of functions belonging to $S_{1}(1, \varepsilon)$, where the sequence $\left(\varepsilon_{j}\right)_{(j \in \Gamma)}$ is only square summable and not summable and who, nevertheless, admit stochastic extensions. Indeed, one will prove in Section 8.3 that, in the proposition below, if the sequence $\left(g_{j}\right)$ is bounded and if the function $V$ is $C^{2}$ with bounded derivatives of order $\leq 2$, then the function $A_{t}(t>0)$ is in $S_{1}(1, \varepsilon)$, with $\varepsilon_{j}=C g_{j}$.

Proposition 8.5. Set $\Gamma=\mathbb{Z}^{d}, H=\ell^{2}\left(\mathbb{Z}^{d}\right)$. Let $B$ be a Banach space such that $(i, H, B)$ is an abstract Wiener space. Let $V: \mathbb{R} \rightarrow \overline{\mathbb{R}}_{+}$be a lipschitzian function. Let $\left(g_{j}\right)_{(j \in \Gamma)}$ be a square summable sequence of positive real numbers such that, if $|j-k| \leq 1$, the quotient $g_{j} / g_{k}$ is smaller than a constant $C>0$. For all $x$ in $H$, set:

$$
p(x)=\sum_{\substack{(j, k) \in \Gamma \times \Gamma \\|j-k|=1}} g_{j} g_{k} V\left(x_{j}-x_{k}\right) \quad A_{t}(x)=e^{-t p(x)} \quad(t>0) .
$$

Then the function $A$ admits a stochastic extension $\widetilde{A}_{t}$ in $B$ in the sense of Definition 4.4.

Proof. Let $\left(E_{n}\right)$ be an increasing sequence in $\mathcal{F}(H)$, whose reunion is dense in $H$. Let $\widetilde{\pi}_{E_{n}}$ be the application defined in Proposition 1.1. Let us prove that the sequence $\left(p \circ \widetilde{\pi}_{E_{n}}\right)$ is a Cauchy sequence in $L^{1}\left(B, \mu_{B, h}\right)$. Denoting by $\left(e_{j}\right)_{(j \in \Gamma)}$ the canonical basis of $H$, one has, for all $m$ and $n$ such that $m<n$ and for all $x \in B$ :

$$
\left|p \circ \widetilde{\pi}_{E_{n}}(x)-p \circ \widetilde{\pi}_{E_{m}}(x)\right| \leq \sum_{\substack{(j, k) \in \Gamma \times \Gamma \\|j-k|=1}} g_{j} g_{k}\left|V\left(\ell_{\pi_{E_{n}}\left(e_{j}-e_{k}\right)}(x)\right)-V\left(\ell_{\pi_{E_{m}}\left(e_{j}-e_{k}\right)}(x)\right)\right|
$$

where $\pi_{E_{n}}: H \rightarrow E_{n}$ is the orthogonal projection. If $K$ is a constant such that $|V(x)-V(y)| \leq K|x-y|$ for all real numbers $x$ et $y$, one has:

$$
\left.\int_{B}\left|p \circ \widetilde{\pi}_{E_{n}}(x)-p \circ \tilde{\pi}_{E_{m}}(x)\right| d \mu_{B, h}(x) \leq K \sum_{\substack{(j, k) \in \Gamma \times \Gamma \\|j-k|=1}} g_{j} g_{k} \int_{B} \ell_{\pi_{E_{n}}\left(e_{j}-e_{k}\right)}(x)-\ell_{\pi_{E_{m}}\left(e_{j}-e_{k}\right)}(x)\right) \mid d \mu_{B, h}(x) .
$$


According to (44), one deduces:

$$
\int_{B}\left|p \circ \widetilde{\pi}_{E_{n}}(x)-p \circ \widetilde{\pi}_{E_{m}}(x)\right| d \mu_{B, h}(x) \leq K \sqrt{2 h / \pi} \sum_{\substack{(j, k) \in \Gamma \times \Gamma \\|j-k|=1}} g_{j} g_{k}\left|\pi_{E_{n}}\left(e_{j}-e_{k}\right)-\pi_{E_{m}}\left(e_{j}-e_{k}\right)\right| .
$$

Under our hypotheses, the family $\left(g_{j}\right)_{(j \in \Gamma)}$ is square summable and the sequence of the projections $\pi_{E_{n}}$ converges strongly to the identity. Hence the right term converges to 0 when $m=\inf (m, n)$ goes to infinity. The sequence of functions $\left(p \circ \widetilde{\pi}_{E_{n}}\right)$ is thus a Cauchy sequence in $L^{1}\left(B, \mu_{B, h}\right)$. The same arguments prove that the limit does not depend on the sequence $\left(E_{n}\right)$. One concludes that the sequence $\left(A_{t} \circ \widetilde{\pi}_{E_{n}}\right)$ converges in probability, which proves the proposition.

The following examples of stochastic extensions are concerned with linear, quadratic or harmonic functions.

Proposition 8.6. Let $(i, H, B)$ be an abstract Wiener space satisfying (4). For all $(a, b)$ in the complexified $H_{\mathbf{C}}$, one defines two functions $\varphi$ and $F$ on $H^{2}$ by:

$$
\varphi(x, \xi)=a \cdot x+b \cdot \xi \quad F(x, \xi)=e^{\varphi(x, \xi)} .
$$

These functions have stochastic extensions $\widetilde{\varphi}$ and $\widetilde{F}$ (in the sense of Definition 4.4, with $p=2$ ).

Proof. It suffices to set

$$
\widetilde{\varphi}(x, \xi)=\ell_{a}(x)+\ell_{b}(\xi) \quad \widetilde{F}(X)=e^{\widetilde{\varphi}(X)} .
$$

Let $\left(E_{n}\right)$ be an increasing sequence in $\mathcal{F}(H)$, whose union is dense in $H$. According to (51) and (44) (with $p=2$ ), one has::

$$
\left\|\widetilde{\varphi}-\varphi \circ \widetilde{\pi}_{E_{n}}\right\|_{L^{2}\left(B^{2}, \mu_{B^{2}, h / 2}\right)}^{2}=h\left(\left|a-\pi_{E_{n}} a\right|^{2}+\left|b-\pi_{E_{n}} b\right|^{2}\right) .
$$

Hence $\widetilde{\varphi}$ is really a stochastic extension of $\varphi$ in $L^{2}\left(B^{2}, \mu_{B^{2}, h / 2}\right)$ in the sense of Definition 4.4. Moreover, still according to (51):

$$
\left\|\widetilde{F}-F \circ \widetilde{\pi}_{E_{n}}\right\|_{L^{2}\left(B^{2}, \mu_{B^{2}, h / 2}\right)}^{2}\left|=\int_{B^{2}}\right| e^{\ell_{a}(x)+\ell_{b}(\xi)}-\left.e^{\ell_{\pi_{E_{n}} a}(x)+\ell_{\pi_{E_{n}} b}(\xi)}\right|^{2} d \mu_{B^{2}, h / 2}(x, \xi) .
$$

Using (49) proves that the right term converges to 0 , which proves the proposition.

Proposition 8.7. Let $(i, H, B)$ be an abstract Wiener space satisfying (4). Let $T$ be a positive, self-adjoint, trace-class operator in $H^{2}$. One defines a function $\varphi$ and, for all positive $t$ a function $F_{t}$ on $H^{2}$ by:

$$
\varphi(X)=<T X, X>\quad F_{t}(X)=e^{-t \varphi(X)} \quad X \in H^{2} .
$$

Then the function $\varphi$ has a stochastic extension $\widetilde{\varphi}$ in $L^{2}\left(B^{2}, \mu_{B^{2}, h / 2}\right)$ (in the sense of Definition 4.4). The norm $N_{2}(\widetilde{\varphi})$ defined in (12) is finite. The function $F_{t}$ has a stochastic extension $\widetilde{F}_{t}$, (in the sense of Definition 4.4). For every bounded continuous function $G$, the function $X \rightarrow G(\varphi(X))$ admits a stochastic extension in the sense of Definition 4.4.

Proof. Let $\left(u_{j}\right)_{(j \geq 0)}$ be a Hilbert basis of $H^{2}$ consisting in eigenvectors of $T$ and let $\left(\lambda_{j}\right)$ be the corresponding eigenvalues. Since the family $\left(\lambda_{j}\right)$ is summable, the sequence of functions

$$
S_{n}(X)=\sum_{j=0}^{n} \lambda_{j} \ell_{u_{j}}(X)^{2}
$$

is a Cauchy sequence in $L^{2}\left(B^{2}, \mu_{B^{2}, h / 2}\right)$. This is a consequence of the Wick Theorem (Theorem 4.3). Indeed, one has for all $u$ and $v$ in $H^{2}$, (with $2 p=4$ ),

$$
\int_{B^{2}} \ell_{u}(X)^{2} \ell_{v}(X)^{2} d \mu_{B^{2}, h / 2}(X)=\frac{h^{2}}{4}\left[|u|^{2}|v|^{2}+2(u \cdot v)^{2}\right] .
$$


The limit of this sequence of functions is denoted by

$$
\widetilde{\varphi}(X)=\sum_{j \geq 0} \lambda_{j} \ell_{u_{j}}(X)^{2} .
$$

One defines a measurable bounded function by:

$$
\widetilde{F}_{t}(X)=e^{-t \widetilde{\varphi}(X)} .
$$

Let $\left(E_{n}\right)$ be an increasing sequence in $\mathcal{F}(H)$, whose union is dense in $H$. According to (51):

$$
\varphi \circ \widetilde{\pi}_{E_{n}}(X)=\sum_{j \geq 0} \lambda_{j}\left(\ell_{\pi_{E_{n}}\left(u_{j}\right)}(X)\right)^{2}
$$

Hence

$$
\begin{gathered}
\left\|\widetilde{\varphi}-\varphi \circ \widetilde{\pi}_{E_{n}}\right\|_{L^{2}\left(B^{2}, \mu_{B^{2}, h / 2}\right)}^{2} \leq \sum\left|A_{j k}^{(n)}\right|\left|\lambda_{j} \lambda_{k}\right| \\
A_{j k}^{(n)}=\int_{B^{2}}\left[\ell_{u_{j}}(X)^{2}-\ell_{\pi_{E_{n}}\left(u_{j}\right)}(X)^{2}\right]\left[\ell_{u_{k}}(X)^{2}-\ell_{\pi_{E_{n}}\left(u_{k}\right)}(X)^{2}\right] d \mu_{B^{2}, h / 2}(X) .
\end{gathered}
$$

Using the particular case (125) of Wick's theorem one gets:

$$
A_{j k}^{(n)}=\frac{h^{2}}{4}\left[1+2\left(u_{j} \cdot u_{k}\right)^{2}+\left|\pi_{E_{n}}\left(u_{j}\right)\right|^{2}\left|\pi_{E_{n}}\left(u_{k}\right)\right|^{2}-2\left(u_{j} \cdot \pi_{E_{n}}\left(u_{k}\right)\right)^{2}-\left|\pi_{E_{n}}\left(u_{j}\right)\right|^{2}-\left|\pi_{E_{n}}\left(u_{k}\right)\right|^{2}\right]
$$

The sequence $A_{j k}^{(n)}$ is thus uniformly bounded and converges to 0 when $n$ goes to infinity, for all fixed $j$ and $k$. Hence if $T$ is trace-class,

$$
\lim _{n \rightarrow+\infty}\left\|\widetilde{\varphi}-\varphi \circ \widetilde{\pi}_{E_{n}}\right\|_{L^{2}\left(B^{2}, \mu_{B^{2}, h / 2}\right)}^{2}=0 .
$$

Therefore $\widetilde{\varphi}$ is really a stochastic extension of $\varphi$ in $L^{2}\left(B^{2}, \mu_{B^{2}, h / 2}\right)$. One deduces easily that, if $\varphi \geq 0$ the function $\widetilde{F}$ is a stochastic extension of $F$ in the sense of Definition 4.4 ,

A necessary condition for a function $f$, continuous on $H$, to admit a stochastic extension in the $L^{2}\left(B, \mu_{B, h}\right)$ sense (cf. Definition 4.4), is that there exists a positive $M$ such that for all subspace $E$ in $\mathcal{F}(H)$ :

$$
\left\|f \circ \tilde{\pi}_{E}\right\|_{L^{2}\left(B, \mu_{B, h}\right)} \leq M .
$$

According to (7), it is equivalent to the fact that, for every subspace $E$ in $\mathcal{F}(H)$, one has:

$$
\left\|\left.f\right|_{E}\right\|_{L^{2}\left(E, \mu_{E, h}\right)} \leq M
$$

But this condition is not sufficient.

Theorem 8.8. Let $(i, H, B)$ be an abstract Wiener space. Let $f$ be a continuous function on $H$. Suppose that the restrictions of $f$ to the subspaces in $\mathcal{F}(H)$ are harmonic functions. Suppose there exist $h>0$ and $M>0$ such that, for every subset $E$ in $\mathcal{F}(H)$, (126) holds. Then $f$ admits a stochastic extension in the $L^{2}\left(B, \mu_{B, h}\right)$ sense.

Proof. For every subspace $E$ in $\mathcal{F}(H)$, set $f_{E}=f \circ \tilde{\pi}_{E}$. One notices that, for all $E$ and $F$ in $\mathcal{F}(H)$, such that $E \subset F$ and for every bounded and measurable function $g: B \rightarrow \mathbf{C}$, one has

$$
\int_{B}\left[f_{F}(x)-f_{E}(x)\right] g \circ \tilde{\pi}_{E}(x) d \mu_{B, h}(x)=0 .
$$

In order to prove it, let us take an orthonormal basis $\left\{e_{1}, \ldots, e_{n}\right\}$ of $F$, such that $\left\{e_{1}, \ldots, e_{m}\right\}$ is a basis of $E$ $(m<n)$. Set $x^{\prime}=\left(x_{1}, x_{m}\right), x^{\prime \prime}=\left(x_{m+1}, \ldots x_{n}\right), x=\left(x^{\prime}, x^{\prime \prime}\right)$ and:

$$
\varphi_{1}\left(x^{\prime}\right)=f\left(x_{1} e_{1}+\ldots+x_{m} e_{m}\right) \quad \varphi_{2}(x)=f\left(x_{1} e_{1}+\ldots+x_{n} e_{n}\right),
$$




$$
\psi\left(x^{\prime}\right)=g\left(x_{1} e_{1}+\ldots+x_{m} e_{m}\right) .
$$

According to (7), the left term of (128) can be written as:

$$
I\left(\varphi_{1}, \varphi_{2}, \psi\right)=\int_{\mathbb{R}^{n}}\left[\varphi_{2}(x)-\varphi_{1}\left(x^{\prime}\right)\right] \psi\left(x^{\prime}\right) d \mu_{\mathbb{R}^{n}, h}(x) .
$$

Since the function $\varphi_{2}$ is harmonic, one has for all $x^{\prime}$, according to the mean-value property:

$$
\int_{\mathbb{R}^{(n-m)}} \varphi_{2}\left(x^{\prime}, x^{\prime \prime}\right) d \mu_{\mathbb{R}^{(n-m)}, h}\left(x^{\prime \prime}\right)=\varphi_{2}\left(x^{\prime}, 0\right)=\varphi_{1}\left(x^{\prime}\right) .
$$

Hence $I\left(\varphi_{1}, \varphi_{2}, \psi\right)=0$ and the property is satisfied. Since $f_{E}$ is $\tilde{\pi}_{E^{-}}$measurable, it amounts to the fact that $f_{E}$ is the conditional expectancy of $f_{F}$ with respect to $\tilde{\pi}_{E}$ :

$$
f_{E}=E\left(f_{F} \mid \tilde{\pi}_{E}\right) .
$$

Let $\left(E_{n}\right)$ be an increasing sequence in $\mathcal{F}(H)$, whose union is dense in $H$. Then the sequence of $\sigma$-algebras $\sigma\left(\tilde{\pi}_{E_{n}}\right)$ is a filtration. The equality (129), with $E$ and $F$ replaced with $E_{n}$ and $E_{n+1}$, proves that the sequence of functions $f_{E_{n}}$ is a martingale for this filtration. Moreover, according to (126), it is bounded in $L^{2}$. There exists a function $\widetilde{f}$ in $L^{2}\left(B, \mu_{B, h}\right)$ (depending, at first sight, on the sequence $\left.\left(E_{n}\right)_{n \in \mathbb{N}}\right)$ such that, for all $n$ :

$$
f_{E_{n}}=E\left(\widetilde{f} \mid \tilde{\pi}_{E_{n}}\right) \quad \quad \lim _{n \rightarrow+\infty}\left\|f_{E_{n}}-\widetilde{f}\right\|_{L^{2}\left(B, \mu_{B, h}\right)}=0 .
$$

The second point shows that the conditions of Definition 4.4 are satisfied.

Now let us check that the function $\widetilde{f}$ constructed above does not depend on the sequence $\left(E_{n}\right)_{n \in \mathbb{N}}$. Consider two increasing sequences $\left(E_{n}\right)$ and $\left(E_{n}^{\prime}\right)$ in $\mathcal{F}(H)$, whose union is dense in $H$ and let $\widetilde{f}$ and $\widetilde{f}^{\prime}$ in $L^{2}\left(B, \mu_{B, h}\right)$ be the functions given by the preceding construction. We have to prove that $\widetilde{f}=\widetilde{f}^{\prime}$. Set $E_{n}^{\prime \prime}=E_{n}+E_{n}^{\prime}$. Let $\tilde{f}^{\prime \prime}$ be the function associated with the sequence $\left(E_{n}^{\prime \prime}\right)$, according to the first step. We first prove that, for every $n$,

$$
E\left(\tilde{f}^{\prime \prime}-\widetilde{f} \mid \tilde{\pi}_{E_{n}}\right)=0 .
$$

According to the point (130) of the first step, one has, for all $n, E\left(\widetilde{f}^{\prime \prime} \mid \tilde{\pi}_{E_{n}^{\prime \prime}}\right)=f_{E_{n}^{\prime \prime}}$. According to (129), one deduces that, since $E_{n} \subset E_{n}^{\prime \prime}$ :

$$
E\left(\tilde{f}^{\prime \prime} \mid \tilde{\pi}_{E_{n}}\right)=E\left(f_{E_{n}^{\prime \prime}} \mid \tilde{\pi}_{E_{n}}\right)=f_{E_{n}} .
$$

But the limit $\tilde{f}$ of (130) is the function associated with the sequence $\left(E_{n}\right)$ by the first step. Hence

$$
E\left(\tilde{f} \mid \tilde{\pi}_{E_{n}}\right)=f_{E_{n}} .
$$

Therefore, for all $n$, (131) is satisfied. With the notations of the first part of the proof, one obtains that, for every bounded function $\varphi: B \rightarrow \mathbb{R}$, measurable with respect to the sub- $\sigma$-algebra $\sigma\left(\tilde{\pi}_{E_{n}}\right)$, one can write:

$$
\int_{B}\left(\widetilde{f}^{\prime \prime}-\widetilde{f}\right) \varphi(x) d \mu_{B, h}(x)=0 .
$$

Set $\mathcal{C}=\cup_{n=1}^{\infty} \sigma\left(\tilde{\pi}_{E_{n}}\right)$ and let $\mathcal{T}$ be the $\sigma$-algebra generated by $\mathcal{C}$. Equation (132) still holds for all $\mathcal{T}$-measurable and bounded functions, according to an consequence of the $\pi \lambda$ theorem of Dynkin. Moreover, one can see that $\mathcal{T}^{*}$, the $\sigma$-algebra generated by $\mathcal{T}$ and the negligible Borel sets, is indeed the Borel $\sigma$-algebra. This establishes (132) for all bounded Borel functions $\varphi$ and implies that $\widetilde{f}^{\prime \prime}=\widetilde{f}$.

\subsection{Examples of symbols in $S_{m}(M, \varepsilon)$.}

Let us now give examples of symbols belonging to the sets $S_{m}(M, \varepsilon)$. The example below shows that Definition 1.1 is coherent with former articles. 
Proposition 8.9. Let $(i, H, B)$ be an abstract Wiener space satisfying (4). For all $a$ and $b$ in $H$, let $F_{a, b}$ be the function on $H^{2}$ defined by:

$$
F_{a, b}(x, \xi)=e^{i(a \cdot x+b \cdot \xi)} .
$$

If $\left(e_{j}\right)_{(j \geq 1)}$ is an arbitrary Hilbert basis of $H$ and if $m$ is a positive integer, the function $F_{a, b}$ is in the set $S_{m}(1, \varepsilon)$ associated with this basis, with

$$
\varepsilon_{j}=\max \left(\left|e_{j} \cdot a\right|,\left|e_{j} \cdot b\right|\right)
$$

and this sequence is square summable. Let $O p_{h}^{W e y l}\left(F_{a, b}\right)$ be the operator, bounded on $L^{2}\left(B, \mu_{B, h / 2}\right)$, associated with $F_{a, b}$ by Theorem 1.4 (this function admits a stochastic extension according to Proposition 8.6). Then the operator $O p_{h}^{W e y l}\left(F_{a, b}\right)$ is equal to the operator $U_{a, b, h}$ defined by

$$
\left(U_{a, b, h} f\right)(u)=e^{-\frac{h}{2}|b|^{2}+i \frac{h}{2} a \cdot b+i \ell_{a+i b}(u)} f(u+h b) \quad \text { a.e.u } \in B .
$$

Proof. The first claim, that $F_{a, b}$ is in $S_{m}(1, \varepsilon)$, is easy to verify. The fact that the right term of (135) defines a bounded operator in $L^{2}\left(B, \mu_{B, h / 2}\right)$ is a consequence of Proposition 4.2. Let us prove that $U_{a, b, h}$ is equal to the operator associated with the function $F_{a, b}$ by Theorem 1.4. Since $F_{a, b}$ is in $S_{2}(1, \varepsilon)$, one can apply (111). Hence it suffices to prove, denoting by $\widetilde{F}_{a, b}$ the stochastic extension of $F_{a, b}$, that, for all $X$ and $Y$ in $H$,

$$
<U_{a, b, h}\left(\widetilde{\Psi}_{X, h}\right), \widetilde{\Psi}_{Y, h}>=\int_{B^{2}} \widetilde{F}_{a, b}(Z) H_{h}^{\text {Gauss }}\left(\widetilde{\Psi}_{X, h}, \widetilde{\Psi}_{Y, h}\right)(Z) d \mu_{B^{2}, h / 2}(Z)
$$

Indeed, the set of functions $\widetilde{\Psi}_{X, h}(X \in H)$ is total in $L^{2}\left(B, \mu_{B, h / 2}\right)$ (this is, for example, a consequence of Janson [J], Theorem D.10). One easily verifies that:

$$
U_{a, b, h}\left(\widetilde{\Psi}_{X, h}\right)=e^{\frac{i}{2}(a \cdot x+b \cdot \xi)} \widetilde{\Psi}_{x-h b, \xi+h a, h} .
$$

Consequently, using (60):

$$
<U_{a, b, h}\left(\widetilde{\Psi}_{X, h}\right), \widetilde{\Psi}_{Y, h}>=e^{-\frac{1}{4 h}\left(|x-y-h b|^{2}+|\xi-\eta+h a|^{2}\right)+\frac{i}{2 h}(y \cdot \xi-x \cdot \eta+h a \cdot(x+y)+h b \cdot(\xi \eta))} .
$$

One then uses the expression of the stochastic extension of the Wigner function of the coherent states, given by (80) and (77), as well as (43). This yields

$$
\begin{aligned}
& \int_{B^{2}} \widetilde{F}_{a, b}(Z) \widetilde{H}_{h}^{\text {Gauss }}\left(\widetilde{\Psi}_{X, h}, \widetilde{\Psi}_{Y, h}\right)(Z) d \mu_{B^{2}, h / 2}(Z)= \\
& =e^{-\frac{1}{4 h}\left(|x-y-h b|^{2}+|\xi-\eta+h a|^{2}\right)+\frac{i}{2 h}(y \cdot \xi-x \cdot \eta+h a \cdot(x+y)+h b \cdot(\xi+\eta))} .
\end{aligned}
$$

One has proved (136). Therefore the operator associated with the function $F_{a, b}$ by Theorem 1.4 is equal to the operator $U_{a, b, h}$ defined by (135).

Proposition 8.10. 1. For all a et $b$ in $H$, the quadratic Weyl form associated (in the sense of Definition 1.2), with the stochastic extension $\widetilde{\varphi}_{a, b}$ of the function $\varphi_{a, b}(x, \xi)=a \cdot x+b \cdot \xi$ satisfies

$$
Q_{h}^{W e y l}\left(\widetilde{\varphi}_{a, b}\right)(f, g)=<\ell_{a+i b} f+\frac{h}{i} b \cdot \frac{\partial f}{\partial u}, g>_{L^{2}\left(B, \mu_{B, h / 2}\right)} \quad f, g \in \mathcal{D},
$$

where the function $u \rightarrow \ell_{a+i b}(u)$ is introduced in Proposition 1.1.

2. Let $F$ be a function on $H^{2}$ which can be expressed as:

$$
F(t, \tau)=\int_{H^{2}} e^{i(x \cdot t+\xi \cdot \tau)} d \nu(x, \xi)
$$


where $\nu$ is a complex measure, bounded on $H^{2}$. Let $\widetilde{F}$ be the stochastic extension of $F$, which exists according to Proposition 8.3. Let $Q_{h}^{W e y l}(\widetilde{F})$ be the quadratic Weyl form associated with this stochastic extension according to Definition 1.2 and $Q_{h}^{A W}(\widetilde{F})$ the quadratic anti-Wick form associated with it according to (23). Then for all $f$ and $g$ in $\mathcal{D}$ :

$$
\begin{gathered}
Q_{h}^{W e y l}(\widetilde{F})(f, g)=\int_{H^{2}}<U_{x, \xi, h} f, g>d \nu(x, \xi), \\
Q_{h}^{A W}(\widetilde{F})(f, g)=\int_{H^{2}} e^{-\frac{h}{4}\left(|x|^{2}+|\xi|^{2}\right)}<U_{x, \xi, h} f, g>d \nu(x, \xi),
\end{gathered}
$$

where $U_{x, \xi, h}$ is defined by (135). There exists an operator $O p_{h}^{W e y l}(F)$ bounded on $L^{2}\left(B, \mu_{B, h / 2}\right)$, whose norm is smaller than $|\nu|\left(H^{2}\right)$, such that $Q_{h}^{W e y l}(\widetilde{F})(f, g)=<O p_{h}^{W e y l}(F) f, g>$ for all $f$ and $g$ in $\mathcal{D}$.

Proof. Point 1). Let us first suppose that $a$ and $b$ belong to $B^{\prime}$. Let $E$ be in $\mathcal{F}\left(B^{\prime}\right)$, let $\widehat{f}$ and $\widehat{g}$ be functions on $E$ such that $f=\widehat{f} \circ \widetilde{\pi}_{E}$ and $g=\widehat{g} \circ \widetilde{\pi}_{E}$. One can assume that $a \in E$ and $b \in E$. Let $\widehat{\varphi}$ be the restriction of $\varphi_{a, b}$ to $E^{2}$. According to (14), one has:

$$
Q_{h}^{W e y l}\left(\widetilde{\varphi}_{a, b}\right)(f, g)=<O p_{h}^{W e y l, L e b}(\widehat{\varphi}) \gamma_{E, h / 2} \widehat{f}, \gamma_{E, h / 2} \widehat{g}>_{L^{2}(E, \lambda)} .
$$

Using the usual Weyl calculus in a finite dimensional setting gives:

$$
O p_{h}^{W e y l, L e b}(\widehat{\varphi}) \gamma_{E, h / 2} \widehat{f}(u)=a \cdot u \gamma_{E, h / 2} \widehat{f}(u)+\frac{h}{i} b \cdot \frac{\partial}{\partial u} \gamma_{E, h / 2} \widehat{f}(u)=\gamma_{E, h / 2} \widehat{F}(u)
$$

where we set:

$$
\widehat{F}(u)=(a+i b) \cdot u \widehat{f}(u)+\frac{h}{i} b \cdot \frac{\partial \widehat{f}}{\partial u} .
$$

Since $\gamma_{E, h / 2}$ is an isometry, one can write:

$$
Q_{h}^{W e y l}\left(\widetilde{\varphi}_{a, b}\right)(f, g)=<\widehat{F}, \widehat{g}>_{L^{2}\left(E, \mu_{E, h / 2}\right)}=<\widehat{F} \circ \widetilde{\pi}_{E}, \widehat{g} \circ \widetilde{\pi}_{E}>_{L^{2}\left(B, \mu_{B, h / 2}\right)},
$$

which implies (137) in the case when $(a, b) \in B^{\prime 2}$.

If $a, b \in H$, let $\left(a_{n}\right)_{n},\left(b_{n}\right)_{n}$ be sequences of $B^{\prime}$ converging to $a, b$ in $H$. One can then check that $N_{1}\left(\tilde{\varphi}_{a_{n}, b_{n}}-\right.$ $\left.\tilde{\varphi}_{a, b}\right) \leq C\left(\left|a-a_{n}\right|+\left|b-b_{n}\right|\right)$ for a real constant $C$. Then Proposition 4.10 implies that $Q_{h}^{W e y l}\left(\widetilde{\varphi}_{a_{n}, b_{n}}\right)(f, g)$ converges to the left term of (137). To treat the right term, one proves that there exists a constant $C(f, g)$ depending on $f$ and $g$ such that

$$
\left|<\ell_{a+i b} f+\frac{h}{i} b \cdot \frac{\partial f}{\partial u}, g>_{L^{2}\left(B, \mu_{B, h / 2}\right)}\right| \leq\left(\left(\left|a-a_{n}\right|+\left|b-b_{n}\right|\right) C(f, g)\right.
$$

using (77) and the fact that $\gamma_{E, h / 2} \hat{f}, \gamma_{E, h / 2} \hat{g}$ are rapidly decreasing. This proves the convergence for the right term and establishes (137) in the general case.

Point 2). Let $E$ be the space of all functions $F$ in $L^{1}\left(B^{2}, \mu_{B^{2}, h / 2}\right)$ such that, for all $Y$ in $H^{2}$, the function $F(Y+\cdot)$ is in $L^{1}\left(B^{2}, \mu_{B^{2}, h / 2}\right)$ and such that $N_{1}(F)$, defined in (12) , is finite. This space, equipped with the norm $N_{1}(\cdot)$, is a Banach space. For all $(x, \xi)$ in $H^{2}$, the function $\widetilde{F}_{x, \xi}$, stochastic extension of the function $F_{x, \xi}$ defined in (133), is in $E$ and the application which associates $\widetilde{F}_{x, \xi}$ with every $(x, \xi)$ in $H^{2}$, is continuous from $H^{2}$ in $\left(E, N_{1}\right)$. More precisely, one can prove that, for $U, V \in H^{2}$ and $Y \in H^{2}$,

$$
\int_{B^{2}}\left|e^{i \ell_{U}(X+Y)}-e^{i \ell_{V}(X+Y)}\right| d \mu_{B^{2}, h / 2}(X)=\int_{\mathbb{R}}\left|e^{i(\sqrt{h / 2}|U-V| u+Y \cdot(U-V))}\right| d \mu_{\mathbb{R}, 1}(u)
$$

and derive from that the continuity. This allows us to define $\int_{H^{2}} \widetilde{F}_{x, \xi} d \nu(x, \xi)$ as a Bochner integral, following Y], chapter V. One can notice that $\int_{H^{2}} \widetilde{F}_{x, \xi} d \nu(x, \xi)$ is the stochastic extension of $(z, \zeta) \mapsto \int_{H^{2}} e^{i(x \cdot z+\xi \cdot \zeta)} d \nu(x, \xi)$ in the sense of Definition 4.4 (for any $\mu_{B^{2}, t}$ and $p=2$ ). 
Finally, for all $f$ and $g$ in $\mathcal{D}$, the application which associates $Q_{h}^{W e y l}(F)(f, g)$ with every $F$ in $E$ is continuous on $E$, according to Proposition 4.10 This yields

$$
Q_{h}^{W e y l}\left(\int_{H^{2}} \widetilde{F}_{x, \xi} d \nu(x, \xi)\right)(f, g)=\int_{H^{2}} Q_{h}^{W e y l}\left(\widetilde{F}_{x, \xi}\right)(f, g) d \nu(x, \xi) .
$$

Since $O p_{h}^{W e y l}\left(F_{x, \xi}\right)$ is the operator $U_{x, \xi, h}$ of (135), one obtains (139). The upper bound comes from the fact that the operator $U_{x, \xi, h}$ is unitary in $L^{2}\left(B, \mu_{B, h / 2}\right)$.

Now let us prove (140). According to (22) and (43), one has the following equality for $(x, \xi) \in\left(B^{\prime}\right)^{2}$ :

$$
\widetilde{H}_{h / 2} \widetilde{F}_{x, \xi}=e^{-\frac{h}{4}\left(|x|^{2}+|\xi|^{2}\right)} \widetilde{F}_{x, \xi} .
$$

Since both sides are continuous functions on $H^{2}$ ( thanks to (49) and Proposition4.5) and valued in $L^{2}\left(B^{2}, \mu_{B^{2}, h / 2}\right)$, it holds true on $H^{2}$. Hence

$$
Q_{h}^{A W}\left(\widetilde{F}_{x, \xi}\right)(f, g)=e^{-\frac{h}{4}\left(|x|^{2}+|\xi|^{2}\right)}<U_{x, \xi, h} f, g>.
$$

Similar arguments allow to integrate on $H^{2}$, which yields (140).

In the articles on Fock spaces, the operator on the right side of (137) is usually called Segal field and its exponential $U_{a, b, h}$, Weyl operator (see [R-S] vol. II, sect. X, p.209-210 or [B-R] vol.II, sect.5.2.1.2, p.12-13).

In B. Lascar's survey LA-1] (Definition 10 and Proposition 1.7), one finds the expression (140) of the antiWick operator for $h=2$ in the case when $F$ is of the form (138). The operator denoted by $\tau_{y^{\prime}+i y^{\prime \prime}}$ in Proposition 1.7 of [LA-1] is equal to $e^{-(1 / 2)\left(\left|y^{\prime}\right|^{2}+\left|y^{\prime \prime}\right|^{2}\right)} U_{y^{\prime \prime}, y^{\prime}, 2}$. To give the analogous expression of the Weyl operator (for $h=2)$, it would have been enough, in $[\mathrm{LA}-1]$, to suppress the factor $e^{-(1 / 2)\left(\left|y^{\prime}\right|^{2}+\left|y^{\prime \prime}\right|^{2}\right)}$ in the integral.

One finds in $\underline{\mathrm{K}-\mathrm{R}}$ an analogous expression, using the creation and annihilation operators. For all $a$ and $b$ in $H$, one denotes by $a(-b+i a)$ and $\left.a^{\star}(-b+i a)\right)$ the operators whose Weyl symbols are respectively the functions on $H^{2}(-b-i a) \cdot(x+i \xi) /(\sqrt{2 h})$ and $(-b+i a)(x-i \xi) /(\sqrt{2 h})$ and which are not bounded on $L^{2}\left(B, \mu_{B h / 2}\right)$. With these notations, one verifies that

$$
U_{a, b, h}=e^{\left.\sqrt{\frac{h}{2}} a^{\star}(-b+i a)\right)} e^{-\sqrt{\frac{h}{2}} a(-b+i a)} e^{-\frac{h}{4}\left(|a|^{2}+|b|^{2}\right)} .
$$

One finds in [K-R] (formula (106), maybe up to some insignificant modifications), when $F$ is of the form (138), an expression comparable to (139) and using the factorization (141) above.

The last example is inspired by interacting lattices. It reminds of a lattice of harmonic oscillators with a coupling between nearest neighbours.

Proposition 8.11. Set $\Gamma=\mathbb{Z}^{d}$ and $H=\ell^{2}\left(\mathbb{Z}^{d}\right)$. Let $V: \mathbb{R} \rightarrow \overline{\mathbb{R}}_{+}$be a smooth function whose derivatives of order greater than 1 are bounded. Let $\left(g_{j}\right)_{(j \in \Gamma)}$ be a sequence of positive real numbers such that, if $|j-k| \leq 1$, the quotient $g_{j} / g_{k}$ is smaller than a constant $K_{0}>1$. For all $(x, \xi)$ in $H^{2}$ and all $t>0$ set:

$$
f(x, \xi)=\sum_{j \in \Gamma} g_{j}^{2} \xi_{j}^{2}+\sum_{\substack{(j, k) \in \Gamma \times \Gamma \\|j-k|=1}} g_{j} g_{k} V\left(x_{j}-x_{k}\right) \quad F_{t}(x, \xi)=e^{-t f(x, \xi)},
$$

where $|\cdot|$ refers to the norm $\ell^{\infty}$ on $\mathbb{R}^{d}$.

For all $m \geq 1$, the function $F_{t}$ is in $S_{m}\left(1, \varepsilon^{(m)}\right)$, with

$$
\varepsilon_{j}^{(m)} \leq C_{m} \max \left(g_{j}^{2}, g_{j}^{1 / m}\right),
$$

where $C_{m}$ depends only on $m, t, d, K_{0}$ and on the bounds for the derivatives of $V$, up to order $2 m$. 
Proof. Let $p$ and $A_{t}$ be the functions defined by (122) (Proposition 8.5), identified with functions on $H^{2}$, independent on $\xi$. With the notations of Definition 1.4, we have, for each finitely supported multi-index $\alpha$ such that $\alpha \neq 0$ and $\alpha_{j} \leq m$ for all $j$ and for all $x \in \mathbb{R}^{n}$ :

$$
\left|\partial_{u}^{\alpha} p(x)\right| \leq \prod_{j \in S(\alpha)}\left(\lambda_{j}^{(m)}\right)^{\alpha_{j}} \quad \lambda_{j}^{(m)}=23^{d} K_{0} \max \left(g_{j}^{2}, g_{j}^{1 / m}\right) \max _{1 \leq k \leq 2 m}\left(\left\|V^{(k)}\right\|_{\infty}\right)^{1 / k}
$$

where $S(\alpha)$ is the set of sites $j \in \Gamma$ such that $\alpha_{j} \neq 0$. We have used the fact that the number of elements of $\mathbb{Z}^{d}$ which are in the unit ball for the $\ell^{\infty}$ norm is lesser than $3^{d}$. We shall use a multidimensional version of the Faa di Bruno formula, due to Constantine Savits [C-S]. For every multi-index $\alpha$, let $F(\alpha)$ be the set of all applications $\varphi$ from the set of multiindices $\beta \neq 0$ such that $\beta \leq \alpha$ into the nonnegative integers such that

$$
\sum_{0 \neq \beta \leq \alpha} \varphi(\beta) \beta=\alpha .
$$

If $\alpha \neq 0$, Constantine-Savits' formula can be written:

$$
\partial_{u}^{\alpha} e^{-t p(x)}=\alpha ! e^{-t p(x)} \sum_{\varphi \in F(\alpha)} \prod_{0 \neq \beta \leq \alpha} \frac{1}{\varphi(\beta) !}\left[\frac{\partial_{u}^{\beta}(-t p(x))}{\beta !}\right]^{\varphi(\beta)} .
$$

In the above sum, we may replace $F(\alpha)$ with the subset $E(\alpha)$ of all $\varphi$ in $F(\alpha)$ such that $\varphi(\beta)=0$ for all multi-index $\beta$ such that $\partial_{u}^{\beta} p(x)$ vanishes identically. By (144) and (145), we have, for each $\varphi \in F(\alpha)$ :

$$
\prod_{0 \neq \beta \leq \alpha}\left|\partial^{\beta}(-t p(x))\right|^{\varphi(\beta)} \leq t^{r(\varphi)} \prod_{j \in S(\alpha)}\left(\lambda_{j}^{(m)}\right)^{\alpha_{j}} \quad r(\varphi)=\sum_{0 \neq \beta \leq \alpha} \varphi(\beta)
$$

If $\alpha_{j} \leq m$ for all $j$, we have $r(\varphi) \leq m|S(\alpha)|$ for each $\varphi \in F(\alpha)$. We have also $\alpha ! \leq(m !)^{|S(\alpha)|}$. For each $\beta \neq 0$ such that $\beta \leq \alpha$ and for each $\varphi$ in $F(\alpha)$, there exists $j$ such that $\beta_{j} \neq 0$, and therefore:

$$
\varphi(\beta) \leq \varphi(\beta) \beta_{j} \leq \sum_{0 \neq \gamma \leq \alpha} \varphi(\gamma) \gamma_{j}=\alpha_{j} \leq m .
$$

Let $I(\alpha)$ be the set of all multi-indices $\beta \neq 0$ such that $\beta \leq \alpha$ and such that $\partial_{u}^{\beta} p$ does not vanish identically. By (148), the number of elements of $E(\alpha)$ is not greater than the number of applications of $I(\alpha)$ to the set $\{0, \ldots, m\}$, that is to say $(m+1)^{|I(\alpha)|}$. By the form (122) of $p$, for each point $j$, the number of multi-indices $\beta$ such that $\beta_{j} \neq 0, \beta_{k} \leq m$ for all $k \in \Gamma$ and such that $\partial^{\beta} p(x)$ does not vanish identically, is smaller than $3^{d} m^{2}$. Therefore, if $\alpha_{k} \leq m$ for all $k$, the number of elements of $I(\alpha)$ is not greater than $3^{d} m^{2}|S(\alpha)|$. Hence the number of elements of $E(\alpha)$ is not greater than $(m+1)^{3^{d} m^{2}|S(\alpha)|}$. Therefore, if $t<1$, and if $\alpha_{k} \leq m$ for all $k$ :

$$
\left|\partial_{u}^{\alpha} e^{-t p(x)}\right| \leq\left[m !(m+1)^{3^{d} m^{2}}\right]^{|S(\alpha)|} \prod_{j \in S(\alpha)}\left(\lambda_{j}^{(m)}\right)^{\alpha_{j}} .
$$

If $t>1$, we have:

$$
\left|\partial_{u}^{\alpha} e^{-t p(x)}\right| \leq t^{m|S(\alpha)|}\left[m !(m+1)^{3^{d} m^{2}}\right]^{|S(\alpha)|} \prod_{j \in S(\alpha)}\left(\lambda_{j}^{(m)}\right)^{\alpha_{j}} .
$$

Therefore, in both cases:

$$
\left|\partial_{u}^{\alpha} e^{-t p(x)}\right| \leq \prod_{j \in S(\alpha)}\left(\varepsilon_{j}^{\prime}(t)\right)^{\alpha_{j}} \quad \quad \varepsilon_{j}^{\prime}(t)=m !(m+1)^{3^{d} m^{2}} \max \left(1, t^{m}\right) \lambda_{j}^{(m)} .
$$

Now set:

$$
q(\xi)=\sum_{j \in \Gamma} g_{j}^{2} \xi_{j}^{2} \quad B_{t}(\xi)=e^{-t q(\xi)} .
$$


One easily checks that:

$$
\left|\partial_{v}^{\beta} e^{-t q(\xi)}\right| \leq \prod_{j \in S(\beta)}\left(\varepsilon_{j}^{\prime \prime}(t)\right)^{\beta_{j}}
$$

with

$$
\varepsilon_{j}^{\prime \prime}(t)=C_{m} g_{j} \sqrt{t} \quad C_{m}=\max _{\alpha \leq m} \sup _{\xi \in \mathbb{R}}\left|\partial^{\alpha} e^{-\xi^{2}}\right|^{1 /|\alpha|} .
$$

The proposition follows, setting $\varepsilon_{j}^{(m)}(t)=\max \left(\varepsilon_{j}^{\prime}(t), \varepsilon_{j}^{\prime \prime}(t)\right)$.

If $H$ is the Hilbert space of Proposition 8.11 let $B$ be a Banach space containing $H$ and such that $(i, H, B)$ is an abstract Wiener space. The space $B$ may be the one of Proposition 8.1 If the family $\left(g_{j}\right)_{(j \in \Gamma)}$ is summable, according to Propositions 8.5 and 8.7, the function $F_{t}$ defined on $H^{2}$ in (142) admits a stochastic extension $\widetilde{F}_{t}$

in $B^{2}$. Moreover, for $m=2$, the family $\left(\varepsilon_{j}^{(2)}\right)_{(j \in \Gamma)}$ satisfying (143) is square summable. According to Theorem 1.4, one can associate with $F_{t}$ a Weyl operator, bounded in $L^{2}\left(B, \mu_{B, h / 2}\right)$.

\section{References}

[A-J-N-1] L. Amour, L. Jager, J. Nourrigat, Bounded Weyl pseudodifferential operators in Fock space, preprint, arXiv:1209.2852, sept. 2012.

[A-J-N-2] L. Amour, L. Jager, J. Nourrigat, On bounded pseudodifferential operators in a high-dimensional setting, preprint, arXiv:1303.1972, march 2013, to appear in Proceedings of the A.M.S.

[B-K] Yu Berezansky, Yu Kondratiev, Spectral methods in infinite dimensional analysis, Kluwer Acad. Publ. 1992.

[Be] F. A. Berezin, The method of second quantization, Translated from the Russian, Pure and Applied Physics, Vol. 24 Academic Press, New York-London 1966.

[B-R] O. Bratteli, D.W. Robinson, Operator algebras and quantum statistical mechanics. 2. Equilibrium states. Models in quantum statistical mechanics. Second edition. Texts and Monographs in Physics. SpringerVerlag, Berlin, 1997.

[B-V] P. Bleher, M. Vishik, On a class of pseudodifferential operators with infinite number of variables and their applications, Math. USSR Sbornik 15, 3, (1971), 443-491.

[C-V] A.P. Calderón, R. Vaillancourt, A class of bounded pseudo-differential operators, Proc. Nat. Acad. Sci. U.S.A, 69, (1972), 1185-1187.

[C-M] R. L. Coifman, Y. Meyer, Au delà des opérateurs pseudo-différentiels, Astérisque, 57, 1978.

[C-R] M. Combescure, D. Robert, Coherent states and applications in mathematical physics, Theoretical and Mathematical Physics. Springer, Dordrecht, 2012.

[C-S] G. M. Constantine, T. H. Savits, A multivariate Faà di Bruno formula with applications, Trans. of the A.M.S, 348, (2), (1996), 503-520.

[C] H. O. Cordes, On compactness of commutators of multiplications and convolutions, and boundedness of pseudo-differential operators, J. Funct. Anal, 18 (1975) 115-131.

[D-H] B. Driver, B. Hall, Yang-Mills theory and the Segal Bargmann transform, Comm. in Math. Phys, 201 (1999) (2) 249-290.

[F] G. B. Folland, Harmonic analysis in phase space. Annals of Mathematics Studies, 122. Princeton University Press, Princeton, NJ, 1989. 
[G-1] L. Gross Measurable functions on Hilbert space, Trans. Amer. Math. Soc. 105 (1962) 372-390.

[G-2] L. Gross, Abstract Wiener spaces, Proc. 5th Berkeley Sym. Math. Stat. Prob, 2, (1965), 31-42.

[G-3] L. Gross, Abstract Wiener measure and infinite dimensional potential theory, in Lectures in modern Analysis and applications, II, Lecture Notes in Math 140, 84-116, Springer (1970).

[G-4] L. Gross, Potential theory on Hilbert space, J. Functional Analysis 1, (1967) 123-181.

[HA] B. Hall, Holomorphic methods in analysis and mathematical physics, First Summer School in Analysis and Mathematical Physics (Cuernavaca Morelos, 1998), 1-59, Contemp. Math., 260, Amer. Math. Soc., Providence, RI, 2000.

[HO] L. Hörmander, The analysis of linear partial differential operators, Volule III, Springer, 1985.

[HW] I. L. Hwang, The $L^{2}$ boundedness of pseudo-differential operators, Trans. Amer. Math. Soc, 302 (1987) $55-76$.

[J] S. Janson, Gaussian Hilbert spaces, Cambridge Tracts in Math. 129, Cambridge Univ. Press (1997).

[K-R] P. Krée, R. Rączka, Kernels and symbols of operators in quantum field theory, Ann. Inst. H. Poincaré Sect. A (N.S.) 28 (1978), no. 1, 41-73.

[K] H. H. Kuo, Gaussian measures in Banach spaces. Lecture Notes in Mathematics, Vol. 463. Springer, BerlinNew York, 1975.

[LA-1] B. Lascar, Equations aux dérivées partielles en dimension infinie. Vector space measures and applications (Proc. Conf., Univ. Dublin, Dublin, 1977), I, pp. 286-313, Lecture Notes in Math, 644, Springer, Berlin, 1978.

[LA-2] B. Lascar, Noyaux d'une classe d'opérateurs pseudo-différentiels sur l'espace de Fock, et applications. Sminaire Paul Krée, 3e année (1976-77), Equations aux dérivées partielles en dimension infinie, Exp. No. $6,43 \mathrm{pp}$.

[LA-3] B. Lascar, Opérateurs pseudo-différentiels en dimension infinie. Etude de l'hypoellipticité de la résolubilité dans des classes de fonctions holderiennes et de distributions pour des opérateurs pseudo-différentiels elliptiques, J. Analyse Math. 33 (1978), 39-104.

[LA-4] B. Lascar, Une classe d'opérateurs elliptiques du second ordre sur un espace de Hilbert, J. Funct. Anal. 35 (1980), no. 3, 316-343.

[LA-5] B. Lascar, Opérateurs pseudo-différentiels en dimension infinie. Applications. C. R. Acad. Sci. Paris Sér. A-B 284 (1977), no. 13, A767-A769,

[LA-6] B. Lascar, Méthodes $L^{2}$ pour des équations aux dérivées partielles dépendant d'une infinité de variables. Séminaire Goulaouic-Schwartz (1975-1976) Equations aux dérivées partielles et analyse fonctionnelle, Exp. No. 5, 11 pp. Centre Math., Ecole Polytech., Palaiseau, 1976.

[LA-7] B. Lascar, Propriétés locales d'espaces de type Sobolev en dimension infinie. Comm. Partial Differential Equations 1 (1976), no. 6, 561-584.

[LA-8] B. Lascar, Propriétés locales d'espaces de type Sobolev en dimension infinie. Séminaire Paul Krée, 1re anne (1974-75), Equations aux dérivées partielles en dimension infinie, Exp. No. 11, 16 pp.

[LA-9] B. Lascar, Opérateurs pseudo-différentiels d'une infinité de variables, d'après M. I. Visik. Séminaire Pierre Lelong (Analyse), Année 1973-1974, pp. 83-90. Lecture Notes in Math., 474, Springer, Berlin, 1975.

[LA-10] B. Lascar, Propriétés d'espaces de Sobolev en dimension infinie, C. R. Acad. Sci. Paris Sér. A-B 280 (1975), no. 23, A1587-A1590. 
[LE] N. Lerner, Metrics on the phase space and non self-adjoint pseudo-differential operators, Birkhäuser Springer, 2010.

[M] P. Malliavin, Stochastic analysis, Grundlehren der Mathematischen Wissenschaften 313. Springer-Verlag, Berlin, 1997.

[N] D. Nualart, Malliavin calculus and its applications, CBMS Regional Conference Series in Mathematics, 110. Published for the Conference Board of the Mathematical Sciences, Washington, DC, by the American Mathematical Society, Providence, RI, 2009.

[RA] R. Ramer, On nonlinear Transformations of Gaussian measures, J. Funct. Analysis, 15 (1974), 166-187.

[R-R] M. Reed, L. Rosen, Support properties of the free measure for Boson fields, Comm. Math. Physics, 36, 123-132 (1974)

[R-S] M. Reed, B. Simon, Methods of modern mathematical physics. II Fourier analysis, self-adjointness Academic Press [Harcourt Brace Jovanovich, Publishers], New York-London (1978)

[SI] B. Simon, The $P(\Phi)_{2}$ Euclidean (Quantum) Field theory, Princeton Series in Physics, Princeton Univ. Press, Princeton (1974).

[SJ] J. Sjöstrand, Singularités analytiques microlocales, Astérisque 95 (1982).

[U-1] A. Unterberger, Oscillateur harmonique et opérateurs pseudo-différentiels, Ann. Inst. Fourier (Grenoble) 29 (1979), no. 3, xi, 201-221.

[U-2] A. Unterberger, Les opérateurs métadifférentiels, in Complex analysis, microlocal calculus and relativistic quantum theory, Lecture Notes in Physics 126 (1980) 205-241.

[Y] K. Yosida, Functional analysis, Reprint of the sixth (1980) edition. Classics in Mathematics. SpringerVerlag, Berlin, 1995.

Laboratoire de Mathématiques, FR CNRS 3399, EA 4535, Université de Reims Champagne-Ardenne, Moulin de la Housse, B. P. 1039, F-51687 Reims, France,

E-mail: laurent.amour@univ-reims.fr

E-mail: lisette.jager@univ-reims.fr

E-mail: jean.nourrigat@univ-reims.fr 